TITLE:

\title{
Semidefinite programming for dynamic steady-state analysis of structures under uncertain harmonic loads
}

\author{
$\operatorname{AUTHOR}(\mathrm{S})$ : \\ Kanno, Yoshihiro; Takewaki, Izuru
}

\section{CITATION:}

Kanno, Yoshihiro ... [et al]. Semidefinite programming for dynamic steady-state analysis of structures under uncertain harmonic loads. Computer Methods in Applied Mechanics and Engineering 2009, 198(41-44): 3239-3261

\section{ISSUE DATE:}

2009-09

URL:

http://hdl.handle.net/2433/89632

\section{RIGHT:}

c 2009 Elsevier Ltd All rights reserved.; この論文は出版社版でありませ ん。引用の際には出版社版をご確認ご利用ください。; This is not the published version. Please cite only the published version. 


\title{
Semidefinite Programming for Dynamic Steady-State Analysis of Structures under Uncertain Harmonic Loads
}

\author{
Yoshihiro Kanno ${ }^{a, \dagger}$, Izuru Takewaki b, \\ ${ }^{a}$ Department of Mathematical Informatics, \\ University of Tokyo, Tokyo 113-8656, Japan \\ ${ }^{\mathrm{b}}$ Department of Urban and Environmental Engineering, \\ Kyoto University, Kyoto 615-8540, Japan
}

\begin{abstract}
This paper presents semidefinite programming approaches for computing confidential bounds for the dynamic steady-state responses of a damped structure subjected to uncertain driving loads. We assume that amplitudes of harmonic driving loads obey a non-probabilistic uncertainty model. The semidefinite programming problems are formulated for finding confidential bounds for various characteristic amounts of dynamic steady-state response, including the modulus and phase angle of the complex amplitude of the displacement and stress. Numerical examples demonstrate that sufficiently tight bounds can be obtained by solving the presented semidefinite programming problems.
\end{abstract}

\section{Keywords}

Semidefinite program; Data uncertainty; Uncertain linear equations; Forced oscillation; Structural dynamics.

\section{Introduction}

Methodologies as well as numerical techniques for robustness and uncertainty analyses have received increasing attention in structural and mechanical design. Since structures built in the real-world always have various uncertainties caused by manufacture errors, limitation of knowledge of input disturbance, observation errors, etc., various approaches have been developed to estimate responses of structures including uncertainties [5, 16, 28-32, 36, 40].

In this paper, we propose a numerical approach to estimate the dynamic structural response under uncertain harmonic loads. There exist two different frameworks with which we consider the

\footnotetext{
${ }^{\dagger}$ Corresponding author. Address: Department of Mathematical Informatics, Graduate School of Information Science and Technology, University of Tokyo, Tokyo 113-8656, Japan. E-mail: kanno@mist.i.u-tokyo.ac.jp. Phone: +81-3-5841-6906, Fax: +81-3-5841-6886.

${ }^{\ddagger}$ Address: Department of Urban and Environmental Engineering, Graduate School of Engineering, Kyoto University, Kyotodaigaku-Katsura, Nishikyo, Kyoto 615-8540, Japan. E-mail: takewaki@archi.kyoto-u.ac.jp. Phone \& Fax: +81-75-383-3294.
} 
uncertain property of a structural system. The one is probabilistic uncertainty modeling and the other non-probabilistic uncertainty modeling. For probabilistic uncertainty models various uncertainty analysis methods have been developed; see, e.g., [36, 41], and the references therein. However, it is often difficult to accurately estimate stochastic parameters which are used in probabilistic structural analyses.

In contrast to probabilistic approaches, the non-probabilistic uncertainty framework treats the uncertain parameters as the so-called unknown-but-bounded parameters, which does not require to estimate the probability distribution of the uncertain parameters. One of well-known approaches with a non-probabilistic uncertainty model is the so-called convex model approach [3, 5, 31, 32, 40]. Note that when we try to estimate a nonlinear function of uncertain parameters, the conventional convex model approaches are valid only if the magnitude of uncertainty is small enough, because it is essentially based on the first-order approximation of the structural response with respect to the uncertain parameters.

As a non-probabilistic decision theory applicable to uncertainties with arbitrary large magnitude, Ben-Haim [4] proposed the info-gap decision theory, and its applications have been found in various fields. In the info-gap decision theory, the robustness function plays a key role which represents the greatest level of uncertainty at which any failure cannot occur. The authors proposed numerical methods for computing the robustness function of structures considering the static response [19] and plastic limit-load factor [25].

For computing the exact bounds of structural responses of a truss including unknown-butbounded uncertainties, mixed integer programming approaches have been developed for the member stress [16] and for the plastic limit-load factor [20].

Concerning uncertainty analysis in structural dynamics, the fuzzy theory has been applied to uncertainty analysis $[12,27]$, which are also regarded as a non-probabilistic approach. For uncertain linear equations (ULE) the interval linear algebra has been well developed [1], which gives a conservative bound for the solution set of the given ULE. The interval algebra has been applied to structural analyses considering uncertainties [29, 30]. Particularly, there have been numerous studies of interval analysis for eigenvalue problems including uncertain parameters [10, 11, 13-15, 24, 26, 33, 34, 37]. These studies are aimed at finding bounds for eigenvalues of structures [10, 11, 13, 15, 24, 26, 33, 34], for eigenmodes [14], modal parameters including the frequency response function [37]. A comparison between the interval analysis and a probabilistic approach for dynamic uncertainty analysis was performed by Qiu and Wang [35].

The convex model approach was also applied to dynamic problems subjected to uncertain impulsive loads [40] and seismic excitations [32], which are assumed to belong to the specified energybounded convex model. Since the modal displacement is a linear function of the excitation, the maximum value of a modal displacement can be bound by using the convex model approach. The maximum values of modal velocity and modal acceleration can be obtained similarly. Then the maximum value of response in the physical coordinates system, e.g. the nodal displacement, is estimated approximately by applying the SRSS or CQC method to the extreme values of modal responses.

For convex optimization problems, including the linear program, semidefinite program (SDP) [17], and second-order cone program (SOCP) [2], etc., a unified methodology of robust optimization was established by Ben-Tal and Nemirovski [7], in which given data in optimization problems are as- 
sumed to have non-probabilistic uncertainties. Calafiore and El Ghaoui [9] proposed a method for finding an ellipsoidal bound for the solution set of ULE by using the SDP relaxation. The authors formulated SDP problems which provides a confidential ellipsoidal bound for static response of uncertain structural systems [18, 21, 22].

In this paper we consider the dynamic response of a damped structure under uncertain driving load. We are particularly interested in the steady state of a forced oscillation induced by a harmonic driving load, where we assume that only the amplitude of driving load has non-probabilistic uncertainties. We attempt to find various bounds for characteristic parameters of the steady state, e.g. lower and upper bounds for the modulus and phase angle of the complex amplitude of nodal displacements.

It is shown in this paper that there exists a close relation between robust optimization in the sense of [7] and bound detection (or, extreme-case detection) problem of an uncertain structural system (see Propositions 3.1, 3.10, and 4.4). If we formulate a bound detection problem directly as an optimization problem, it is often that the resulting optimization problem is nonconvex. Then standard nonlinear programming approaches in general converge to its local optimal solution. Note that such a local optimal solution corresponds to an underestimate of the extreme structural response. In contrast, for the presented robust optimization reformulation of a bound detection, it is shown that any feasible solution corresponds to an overestimate of the extreme structural response. For the purpose of robustness analysis, an overestimate may be more important than an underestimate, provided that the exact extreme value is difficult to obtain. This motivates us to investigate robust optimization problem formulations of various bound detection problems. Thus, this paper sheds a new light on conservative-bound detection of the structural response from the viewpoint of robust optimization.

Based on the the so-called $\mathcal{S}$-lemma [8, section 2.6.3], we next present a sufficient condition, with which the (infinitely many) constraints in the robust optimization formulation stated above are satisfied. Finally, we construct a convex optimization problem which is guaranteed to provide a conservative approximation of the exact optimal solution to the bound detection problem.

This fundamental idea is similar to that used in [22], which discussed an SDP formulation in order to find a confidential ellipsoidal bound for static response of an uncertain structural system. However, in this paper we present different formulations by utilizing particular properties of our specific dynamic problem, i.e. for finding an upper bound for the modulus of the displacement amplitude, we propose an SDP problem which is smaller in comparison with the formulation obtained by applying the method in [22] (see section 3.4.1); for finding a lower bound for the modulus of the displacement amplitude, we present an SOCP problem; and for finding bounds for the phase angle of the displacement amplitude, the method in [22] cannot be applied (see section 3.4.3), and hence we propose a completely new approach.

Although our approach has certain limitations on uncertain situation that can be considered as mentioned above, to the authors' knowledge there has been no efficient method for finding a sufficiently-tight conservative bound for the dynamic response which is expressed as a nonlinear function of the uncertain parameters. For example, our approach finds a bounds for the response in the physical coordinate system directly without using any approximation such as the SRSS method. In addition, since we consider relatively small damping, it is important to estimate the resonant 
behavior caused by the harmonic excitation with a frequency which is close to the fundamental natural frequency. Such an estimate can be obtained efficiently by using our method.

It should be emphasized that our goal formulations for conservative bounds are SDP and SOCP problems. It is known that SDP and SOCP problems can be solved by using the primal-dual interiorpoint method in polynomial time $[2,17]$. Hence, our method can find a bound for the structural response in polynomial time, in contrast to the fact that most of numerical methods based on the interval algebra have in general exponential complexity [6, section 6.5.3].

This paper is organized as follows. Section 2 introduces the governing equation of our problem, as well as a non-stochastic uncertainty model of the dynamic load. In section 3, we formulate SDP problems which give conservative bounds for the modulus and phase angle of the displacement amplitude. In section 4, we particularly consider a nodal displacement vector, and propose further bounds for the norm and ratio of the moduli of the nodal displacement amplitudes. Numerical examples are presented in section 5 for the bound detection problems investigated in the two previous sections. In section 6 , we consider a slightly generalized case of our uncertainty model. Some conclusions are drawn in section 7 . For readability, all proofs of the technical results are collected in appendix A.

We present a few words regarding our notation at first. All vectors are assumed to be column vectors. For $\mathcal{X} \subseteq \mathbb{R}^{n}$ and $\mathcal{Y} \subseteq \mathbb{R}^{m}$, their Cartesian product is defined by $\mathcal{X} \times \mathcal{Y}=\left\{\left(\boldsymbol{x}^{\mathrm{T}}, \boldsymbol{y}^{\mathrm{T}}\right)^{\mathrm{T}} \in\right.$ $\left.\mathbb{R}^{n+m} \mid \boldsymbol{x} \in \mathcal{X}, \boldsymbol{y} \in \mathcal{Y}\right\}$, where ${ }^{\mathrm{T}}$ denotes the transpose of a vector or a matrix. Particularly, we write $\mathbb{R}^{n+m}=\mathbb{R}^{n} \times \mathbb{R}^{m}$. The $(n+m)$-dimensional column vector $\left(\boldsymbol{x}^{\mathrm{T}}, \boldsymbol{y}^{\mathrm{T}}\right)^{\mathrm{T}}$ consisting of $\boldsymbol{x} \in \mathbb{R}^{n}$ and $\boldsymbol{y} \in \mathbb{R}^{m}$ is often written simply as $(\boldsymbol{x}, \boldsymbol{y})$. We denote by $\mathbb{R}_{+}^{n}$ the non-negative orthant defined by $\mathbb{R}_{+}^{n}=\left\{\boldsymbol{x}=\left(x_{i}\right) \in \mathbb{R}^{n} \mid x_{i} \geq 0(i=1, \ldots, n)\right\}$. We write $\boldsymbol{x} \geq \mathbf{0}$ if $\boldsymbol{x} \in \mathbb{R}_{+}^{n}$. We denote by $\mathcal{S}^{n} \subset \mathbb{R}^{n \times n}$ the set of all $n \times n$ real symmetric matrices. We write $A \succeq O$ if $A \in \mathcal{S}^{n}$ is positive semidefinite. It should be clear that $\boldsymbol{x} \geq \boldsymbol{y}$ and $A \succeq B$ mean $\boldsymbol{x}-\boldsymbol{y} \geq \mathbf{0}$ and $A-B \succeq O$, respectively. For a vector $\boldsymbol{x} \in \mathbb{R}^{n}$ we denote by $\|\boldsymbol{x}\|$ the standard Euclidean norm, i.e. $\|\boldsymbol{x}\|=\left(\boldsymbol{x}^{\mathrm{T}} \boldsymbol{x}\right)^{1 / 2}$. We denote by $I_{n}$ the $n \times n$ identity matrix. We also write $I$ instead of $I_{n}$, unless its size is not clear from the context. Let $\operatorname{Re} z$ and $\operatorname{Im} z$ denote the real and imaginary parts of $z \in \mathbb{C}$, respectively. We denote by $|z|$ the modulus, or the absolute value, of $z \in \mathbb{C}$, i.e. $|z|=\left[(\operatorname{Re} z)^{2}+(\operatorname{Im} z)^{2}\right]^{1 / 2}$. We denote by $\operatorname{Arg} z \operatorname{the}$ principal value of the argument of $z \in \mathbb{C}$, which satisfies $-\pi<\operatorname{Arg} z \leq \pi$.

\section{Uncertain equations for steady state vibration}

Consider a finite-dimensional linear elastic structure. Small displacements and small strains are assumed.

\subsection{Governing equations}

Let $d$ denote the number of degrees of freedom of displacements. In this paper we consider the harmonic driving load defined by $\boldsymbol{f} e^{\mathrm{i} \omega t}$, where $\boldsymbol{f} \in \mathbb{R}^{d}$ and $\omega \in \mathbb{R}$. We denote by $K \in \mathcal{S}^{d}, M \in \mathcal{S}^{d}$, and $C \in \mathcal{S}^{d}$ the stiffness, mass and damping matrices, respectively. The d'Alembert principle yields

$$
M \ddot{\hat{\boldsymbol{u}}}+C \dot{\hat{\boldsymbol{u}}}+K \hat{\boldsymbol{u}}=\boldsymbol{f} e^{\mathrm{i} \omega t},
$$


where $\hat{\boldsymbol{u}} \in \mathbb{C}^{d}$ is the displacement vector. By substituting $\hat{\boldsymbol{u}}=\boldsymbol{u} e^{\mathrm{i} \omega t}$ into (1), we find the steady state oscillation, $\boldsymbol{u} \in \mathbb{C}^{d}$, driven by $\boldsymbol{f} e^{\mathrm{i} \omega t}$ as the solution to

$$
\left(-\omega^{2} M+\mathrm{i} \omega C+K\right) \boldsymbol{u}=\boldsymbol{f} .
$$

\subsection{Uncertainty model}

In this section we define a non-probabilistic uncertainty model of the harmonic driving load.

Suppose that the amplitude vector of the load, $\boldsymbol{f}$, in (2) is not known precisely, or is uncertain. Throughout the paper we assume that the uncertainty exists only in $\boldsymbol{f}$, and that $K, M, C$, and $\omega$ in (2) are known precisely.

Let $\tilde{\boldsymbol{f}} \in \mathbb{R}^{d}$ denote the nominal value, or the best estimate, of $\boldsymbol{f}$. We describe the uncertainty of $\boldsymbol{f}$ by using an unknown vector $\boldsymbol{\zeta}$. Assume that $\boldsymbol{f}$ depends on $\boldsymbol{\zeta} \in \mathbb{R}^{k}$ affinely as

$$
f=\tilde{f}+F_{0} \zeta
$$

where $F_{0} \in \mathbb{R}^{d \times k}$ is a constant matrix. Note that the matrix $F_{0}$ represents the relative magnitude of the uncertainty of $f_{j}(j=1, \ldots, d)$ and the relationship of the uncertainties among $f_{1}, \ldots, f_{d}$. See, for an illustrative example, Example 2.1.

Suppose that $\zeta$ satisfies

$$
\alpha \geq\left\|T_{j} \zeta\right\|, \quad j=1, \ldots, \ell,
$$

where $T_{j} \in \mathbb{R}^{m_{j} \times k}(j=1, \ldots, \ell)$ are constant matrices, and $\alpha \in \mathbb{R}_{+}$is constant. Throughout the paper we assume that the set $\left\{\boldsymbol{\zeta} \mid \alpha \geq\left\|T_{j} \boldsymbol{\zeta}\right\|(j=1, \ldots, \ell)\right\}$ is bounded for any given $\alpha \in \mathbb{R}_{+}$. This assumption is satisfied if $T_{1}, \ldots, T_{\ell}$ satisfy

$$
\operatorname{rank}\left[\begin{array}{c}
T_{1} \\
\vdots \\
T_{\ell}
\end{array}\right]=k .
$$

We call $\boldsymbol{\zeta}$ the vector of uncertain parameters, or unknown-but-bounded parameters.

It follows (3) and (4) that the amplitude of driving load, $\boldsymbol{f}$, is running through the set

$$
\mathcal{F}(\alpha)=\left\{\tilde{\boldsymbol{f}}+F_{0} \boldsymbol{\zeta} \mid \alpha \geq\left\|T_{j} \boldsymbol{\zeta}\right\|(j=1, \ldots, \ell)\right\} .
$$

We call $\mathcal{F}(\alpha)$ the uncertainty set of $\boldsymbol{f}$. Recall (2), and then the uncertain linear equations (ULE) that we are interested in are written as

$$
\left(-\omega^{2} M+\mathrm{i} \omega C+K\right) \boldsymbol{u}=\boldsymbol{f}, \quad \boldsymbol{f} \in \mathcal{F}(\alpha) .
$$

Throughout the paper we assume that the equations in (6) have a solution for any $\boldsymbol{f} \in \mathcal{F}$.

In the uncertainty model defined by (5), we see that the greater the value of $\alpha$, the greater the range of possible variation of $\boldsymbol{f}$, and hence we call $\alpha$ the uncertainty parameter. More precisely, $0 \leq \alpha_{1}<\alpha_{2}$ implies $\mathcal{F}\left(\alpha_{1}\right) \subset \mathcal{F}\left(\alpha_{2}\right)$, which meats the nesting axiom in the info-gap theory [4]. As another important property it should be mentioned that our model in (5) satisfies $\mathcal{F}(0)=\{\tilde{\boldsymbol{f}}\}$, which implies that the estimate $\tilde{\boldsymbol{f}}$ is correct at $\alpha=0$. This property is called the contraction axiom 


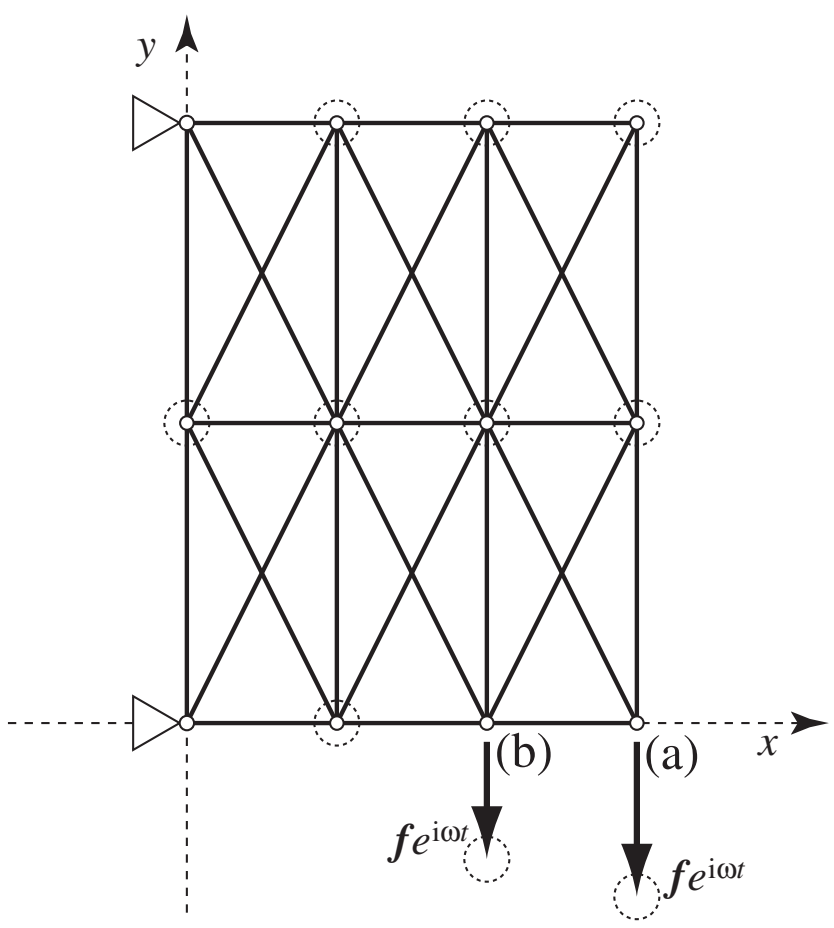

Figure 1: A 29-bar truss subjected to dynamic loading.

in the info-gap theory [4]. In the remainder of the paper we often write $\mathcal{F}$ instead of $\mathcal{F}(\alpha)$, unless the meaning is not clear from the context.

For simple presentation we assume that $F_{0}$ in (5) is nonsingular in sections $3-5$. This assumption implies that all elements, $f_{1}, \ldots, f_{d}$, of $\boldsymbol{f}$ have uncertainties, and that $d=k$. See section 6 for the case in which $F_{0}$ is singular.

Example 2.1. Consider a plane truss shown in Figure 1, where $d=20$. As the nominal load $\tilde{\boldsymbol{f}}$, relatively large forces are applied at the nodes (a) and (b). Suppose that uncertain loads may possibly exist at all free nodes, and then the external forces are independently running through the circles depicted with the dotted lines in Figure 1. Such an uncertainty model can be represented by (5) as follows.

Note that $k=20$, because $F_{0}$ is assumed to be regular. Suppose that all circles in Figure 1 have common range. Then we may put $F_{0}=\bar{f}_{0} I_{20}$. Since we consider 10 independent circles in Figure 1 , put $\ell=10$ and define $T_{j} \in \mathbb{R}^{2 \times 20}(j=1, \ldots, \ell)$ as

$$
T_{1}=\left[\begin{array}{llllll}
1 & 0 & 0 & 0 & \cdots & 0 \\
0 & 1 & 0 & 0 & \cdots & 0
\end{array}\right], \quad T_{2}=\left[\begin{array}{llllll}
0 & 0 & 1 & 0 & \cdots & 0 \\
0 & 0 & 0 & 1 & \cdots & 0
\end{array}\right], \ldots, T_{10}=\left[\begin{array}{lllll}
0 & \cdots & 0 & 1 & 0 \\
0 & \cdots & 0 & 0 & 1
\end{array}\right]
$$

Consequently, (5) with these $T_{j}$ represents the uncertainty model illustrated in Figure 1, where the nodal force at each node may perturb in the circle with the radius $\alpha \bar{f}_{0}$.

Example 2.2. Consider the uncertainty model investigated in Example 2.1. Now we suppose that the uncertain forces are running through squares instead of circles illustrated in Figure 1. Such an uncertainty model is called the interval uncertainty model and widely used in uncertainty analysis of structures $[10,11,29,30]$. Let $F_{0}$ in (5) be a diagonal matrix, where $d=k=20$. Since we 
consider 20 independent intervals, put $\ell=20$ and define $T_{1}, \ldots, T_{\ell} \in \mathbb{R}^{1 \times 20}$ by $T_{j}=\boldsymbol{e}_{j}^{\mathrm{T}}$, where $\boldsymbol{e}^{j}$ denote the $j$ th column vector of the $20 \times 20$ identity matrix. Thus (5) includes the conventional interval uncertainty model as a particular case.

Note again that uncertain forces can exist at all free nodes in the uncertainty models studied in Example 2.1 and Example 2.2, because $F_{0}$ is nonsingular. In section 6 we suppose that uncertain forces can exist only at specified nodes, in which $F_{0}$ is singular.

\section{Bounds for complex amplitude}

For the given $\boldsymbol{f} \in \mathcal{F}$, let $\boldsymbol{u}(\boldsymbol{f}) \in \mathbb{C}^{d}$ denote a solution (complex number) to (2). We denote by $u_{q}(\boldsymbol{f})$ the $q$ th element of $\boldsymbol{u}(\boldsymbol{f})$. In this section, we attempt to find the bounds for $\left|u_{q}\right|$ and $\operatorname{Arg} u_{q}$ when $\boldsymbol{f} \in \mathcal{F}$. More precisely, the problem in this study is stated as follows: given the ULE (6), find $\bar{r}, \underline{r}$, $\bar{\theta}$, and $\underline{\theta} \in \mathbb{R}$ satisfying

$$
\begin{aligned}
& \underline{r} \leq\left|u_{q}(\boldsymbol{f})\right| \leq \bar{r}, \quad \forall \boldsymbol{f} \in \mathcal{F}(\alpha), \\
& \underline{\theta} \leq \operatorname{Arg} u_{q}(\boldsymbol{f}) \leq \bar{\theta}, \quad \forall \boldsymbol{f} \in \mathcal{F}(\alpha) .
\end{aligned}
$$

Such a set of bounds is not unique in general. Naturally we are interested in a set of bounds which is as tight as possible.

In this section we assume that $F_{0}$ in (5) is regular, and hance $k=d$. A formulation that works without this assumption will be given in section 6 .

\subsection{Upper bound for modulus of displacement amplitude}

The maximum value, $r_{\max }$, of $\left|u_{q}\right|$ is defined by

$$
r_{\max }=\max _{\boldsymbol{f}}\left\{\left|u_{q}(\boldsymbol{f})\right|: \boldsymbol{f} \in \mathcal{F}\right\} .
$$

In this section we formulate an optimization problem in order to obtain an upper bound of $r_{\max }$, i.e. $\bar{r} \in \mathbb{R}$ satisfying $\bar{r} \geq r_{\max }$. Certainly we are interested in finding a small upper bound $\bar{r}$ as far as possible.

Observe that (2) is rewritten as

$$
\left[\begin{array}{cc}
-\omega^{2} M+K & -\omega C \\
\omega C & -\omega^{2} M+K
\end{array}\right]\left[\begin{array}{l}
\operatorname{Re} \boldsymbol{u} \\
\operatorname{Im} \boldsymbol{u}
\end{array}\right]=\left[\begin{array}{l}
\boldsymbol{f} \\
\mathbf{0}
\end{array}\right] .
$$

For $\boldsymbol{u} \in \mathbb{C}^{d}$, define $\boldsymbol{v} \in \mathbb{R}^{2 d}$ by

$$
\boldsymbol{v}=\left[\begin{array}{c}
\operatorname{Re} \boldsymbol{u} \\
\operatorname{Im} \boldsymbol{u}
\end{array}\right]
$$

Then (8) is rewritten simply as

$$
\begin{aligned}
& S_{1} \boldsymbol{v}=\boldsymbol{f}, \\
& S_{2} \boldsymbol{v}=\mathbf{0},
\end{aligned}
$$




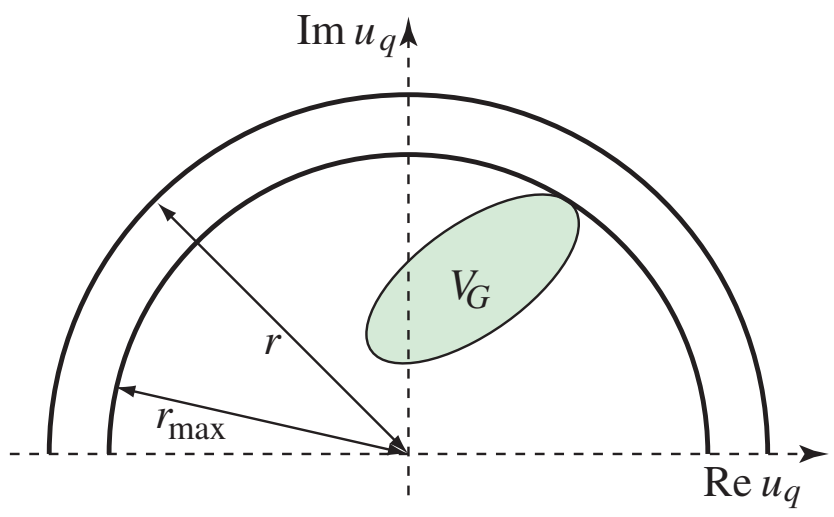

Figure 2: A circle providing an upper bound of $\left|u_{q}\right|$.

where $S_{1}, S_{2} \in \mathbb{R}^{d \times 2 d}$ are defined by

$$
\begin{aligned}
& S_{1}=\left[\begin{array}{ll}
K-\omega^{2} M & -\omega C
\end{array}\right], \\
& S_{2}=\left[\begin{array}{ll}
\omega C & K-\omega^{2} M
\end{array}\right] .
\end{aligned}
$$

Define the set $\mathcal{V} \subseteq \mathbb{R}^{2 d}$ by

$$
\mathcal{V}=\left\{\boldsymbol{v} \mid S_{1} \boldsymbol{v}=\boldsymbol{f}, S_{2} \boldsymbol{v}=\mathbf{0}, \boldsymbol{f} \in \mathcal{F}\right\}
$$

i.e. $\mathcal{V}$ is the set of all possible solutions to the system of equations, (10) and (11), when $\boldsymbol{f}$ is running through the uncertainty set $\mathcal{F}$. Note that $\mathcal{V}$ is related to the solution set to ULE (6) as

$$
\mathcal{V}=\left\{\left[\begin{array}{l}
\operatorname{Re} \boldsymbol{u} \\
\operatorname{Im} \boldsymbol{u}
\end{array}\right] \mid\left(-\omega^{2} M+\mathrm{i} \omega C+K\right) \boldsymbol{u}=\boldsymbol{f}, \boldsymbol{f} \in \mathcal{F}\right\} .
$$

Let $G \in \mathbb{R}^{2 \times 2 d}$ denote a constant matrix satisfying

$$
G \boldsymbol{v}=\left[\begin{array}{c}
\operatorname{Re} u_{q} \\
\operatorname{Im} u_{q}
\end{array}\right]
$$

which yields

$$
\left|u_{q}\right|=\|G \boldsymbol{v}\|
$$

It should be clear that $\|G \boldsymbol{v}\|$ denotes the standard Euclidean norm of the real vector $G \boldsymbol{v} \in \mathbb{R}^{2}$.

We first reformulate the optimization problem (7) into the form similar to the robust optimization [7]. Our reformulation is based on the following idea. Assume that $g: \mathbb{R}^{n} \rightarrow \mathbb{R}$ is bounded above on $\mathcal{X} \subseteq \mathbb{R}^{n}$. Then we can show the relation

$$
\max _{\boldsymbol{x}}\{g(\boldsymbol{x}): \boldsymbol{x} \in \mathcal{X}\}=\min _{t}\{t: t \geq g(\boldsymbol{x})(\forall \boldsymbol{x} \in \mathcal{X})\},
$$

because $t$ is regarded as an upper bound of $g(\boldsymbol{x})$ if $t$ satisfies $t \geq g(\boldsymbol{x})(\forall \boldsymbol{x} \in \mathcal{X})$. By applying this idea to (7) we obtain the following proposition. 
Proposition 3.1. $r_{\max }$ defined in (7) satisfies

$$
r_{\max }=\min _{r}\{r: r \geq\|G \boldsymbol{v}\|(\forall \boldsymbol{v} \in \mathcal{V})\} .
$$

Note that all proofs in this section appear in appendix A.1.

Remark 3.2. The assertion of Proposition 3.1 is interpreted geometrically as follows. In Figure 2, we define the set $\mathcal{V}_{G} \subset \mathbb{R}^{2}$ by $\mathcal{V}_{G}=\{G \boldsymbol{v} \mid \boldsymbol{v} \in \mathcal{V}\}$ for exposition. In the problem (14), we attempt to find a point $v_{G} \in \mathcal{V}_{G}$ which attains the maximum Euclidean distance from the origin. In contrast, in the problem (14), we consider a circle which includes $\mathcal{V}_{G}$ and is centered at the origin, and attempt to minimize the radius of such a circle. Then the minimum radius becomes equivalent to $r_{\max }$.

In this paper we are particularly interested in dealing with the optimization problem in the form of (14), which is the form of robust optimization problems introduced in [7]. Similar formulations for various bound-detection problems are found in (26), (31), (49), and (52). The advantage of such a reformulation is found as follows. Since the original bound-detection problem (7) is nonconvex, standard nonlinear programming approaches in general converge to its local optimal solution, and there exists no proof of global optimality for the obtained solution. Hence, the obtained solution is generically a lower bound for $r_{\max }$. In contrast, if we can find a feasible solution of (14), then it is guaranteed that such a solution corresponds to an upper bound of $r_{\max }$. Since we are interested in the maximum value, $r_{\max }$, of $\left|u_{q}\right|$, an upper bound has more importance than lower bounds from the practical point of view. This is the reason why the reformulation of (7) into (14) is important for finding a conservative bound. The relation between those two kinds of problems (see Propositions 3.1, 3.10, and 4.4) presented in this paper sheds a new light on conservative-bound detection of the structural response from the viewpoint of robust optimization.

It is difficult to solve the problem (14) directly, since it has an infinite number of constraint conditions. In this paper we attempt to replace such an intractable constraint condition with a tractable one which is guaranteed to be conservative. The basic idea with which we can address our tractable and conservative reformulation is stated in the following proposition.

Proposition 3.3. Consider two conditions:

$$
\begin{array}{ll}
\text { (a) : } & {\left[\begin{array}{l}
\boldsymbol{x} \\
1
\end{array}\right]^{\mathrm{T}} Q_{i}\left[\begin{array}{l}
\boldsymbol{x} \\
1
\end{array}\right] \geq 0(i=1, \ldots, m), \quad\left[\begin{array}{c}
\boldsymbol{x} \\
1
\end{array}\right]^{\mathrm{T}} P_{j}\left[\begin{array}{c}
\boldsymbol{x} \\
1
\end{array}\right]=0(j=1, \ldots, k) ;} \\
\text { (b) : }\left[\begin{array}{c}
\boldsymbol{x} \\
1
\end{array}\right]^{\mathrm{T}} Q_{0}\left[\begin{array}{l}
\boldsymbol{x} \\
1
\end{array}\right] \geq 0 .
\end{array}
$$

The implication

$$
\text { (a) } \Rightarrow \text { (b) }
$$

holds if there exist $\tau_{1}, \ldots, \tau_{m}$ and $\sigma_{1}, \ldots, \sigma_{k}$ satisfying

$$
\begin{aligned}
& Q_{0} \succeq \sum_{i=1}^{m} \tau_{i} Q_{i}+\sum_{j=1}^{k} \sigma_{j} P_{j}, \\
& \tau_{1}, \ldots, \tau_{m} \geq 0 .
\end{aligned}
$$


Observe that the conditions (a) and (b) in Proposition 3.3 are represented by some quadratic inequalities. In the following proposition, we eliminate the uncertain parameters $\zeta$ from the condition $\boldsymbol{v} \in \mathcal{V}$ which appears in (14), and obtain a finite number of quadratic inequalities.

Proposition 3.4. The set $\mathcal{V}$ defined in (12) can be represented by some quadratic inequalities of $\boldsymbol{v}$. That is, $\boldsymbol{v} \in \mathcal{V}$ if and only if $\boldsymbol{v}$ satisfies

$$
\begin{aligned}
& {\left[\begin{array}{l}
\boldsymbol{v} \\
1
\end{array}\right]^{\mathrm{T}} \Psi_{j}\left[\begin{array}{l}
\boldsymbol{v} \\
1
\end{array}\right] \geq 0, \quad j=1, \ldots, \ell,} \\
& {\left[\begin{array}{l}
\boldsymbol{v} \\
1
\end{array}\right]^{\mathrm{T}} \Theta\left[\begin{array}{l}
\boldsymbol{v} \\
1
\end{array}\right]=0,}
\end{aligned}
$$

where $\Psi_{j}(j=1, \ldots, \ell)$ and $\Theta$ are constant matrices defined by

$$
\begin{aligned}
& \Psi_{j}=\operatorname{diag}\left(\mathbf{0}, \alpha^{2}\right)-\left[\begin{array}{c}
S_{1}^{\mathrm{T}} \\
-\tilde{\boldsymbol{f}}^{\mathrm{T}}
\end{array}\right]\left(T_{j} F_{0}^{-1}\right)^{\mathrm{T}} T_{j} F_{0}^{-1}\left[\begin{array}{ll}
S_{1} & -\tilde{\boldsymbol{f}}
\end{array}\right], \quad j=1, \ldots, \ell, \\
& \Theta=-\left[\begin{array}{c}
S_{2}^{\mathrm{T}} \\
O
\end{array}\right]\left[\begin{array}{ll}
S_{2} & O
\end{array}\right] .
\end{aligned}
$$

Proposition 3.4 implies that the condition $\boldsymbol{v} \in \mathcal{V}$ can be rewritten as a finite number of quadratic inequalities in terms of $\boldsymbol{v}$. By applying Proposition 3.3, we construct a sufficient condition for the constraint condition of the problem (14) in the following proposition.

Proposition 3.5. $r \in \mathbb{R}_{+}$satisfies the condition

$$
\forall \boldsymbol{v} \in \mathcal{V}: \quad r \geq\|G \boldsymbol{v}\|
$$

if there exists a vector $(\boldsymbol{w}, s) \in \mathbb{R}_{+}^{\ell} \times \mathbb{R}$ satisfying

$$
\left[\begin{array}{cc}
-G^{\mathrm{T}} G & \mathbf{0} \\
\mathbf{0}^{\mathrm{T}} & r^{2}
\end{array}\right] \succeq \sum_{j=1}^{\ell} w_{j} \Psi_{j}+s \Theta
$$

Proposition 3.5 presents a sufficient condition for the constraint condition of the problem (14). This naturally leads us to construct the following problem which yields a conservative bound for $r_{\max }$.

Proposition 3.6. Define $\rho^{*}$ by

$$
\rho^{*}=\min _{\rho, \boldsymbol{w}, s}\left\{\rho:\left[\begin{array}{cc}
-G^{\mathrm{T}} G & \mathbf{0} \\
\mathbf{0}^{\mathrm{T}} & \rho
\end{array}\right]-\sum_{j=1}^{\ell} w_{j} \Psi_{j}-s \Theta \succeq O, \boldsymbol{w} \geq \mathbf{0}\right\} .
$$

Then $r_{\max }^{2} \leq \rho^{*}$ holds for $r_{\max }$ defined in $(7)$, i.e. $\sqrt{\rho^{*}}$ corresponds to an upper bound of $\left|u_{q}\right|$.

Since (19) is an SDP problem [17] in the variables $\rho \in \mathbb{R}, \boldsymbol{w} \in \mathbb{R}^{\ell}$, and $s \in \mathbb{R}$, we can obtain $\rho^{*}$ efficiently by using the primal-dual interior-point method. 


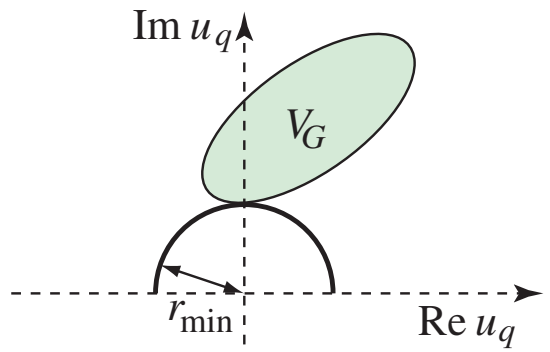

Figure 3: A circle providing the minimum value of $\left|u_{q}\right|$.

\subsection{Lower bound for modulus of displacement amplitude}

In a manner similar to (7), the minimum value, $r_{\min }$, of $\left|u_{q}\right|$ is defined by

$$
r_{\min }=\min _{\boldsymbol{f}}\left\{\left|u_{q}(\boldsymbol{f})\right|: \boldsymbol{f} \in \mathcal{F}(\alpha)\right\} .
$$

Figure 3 depicts the definition of $r_{\min }$. In contrast to the case of $r_{\max }$ investigated in section 3.1, the following proposition shows that we can reformulate the problem (20) directly as a convex optimization problem.

Proposition 3.7. Define $t^{*}$ by

$$
t^{*}=\min _{t, \boldsymbol{v}, \boldsymbol{\zeta}}\left\{t: r \geq\|G \boldsymbol{v}\|, S_{1} \boldsymbol{v}=\tilde{\boldsymbol{f}}+F_{0} \boldsymbol{\zeta}, S_{2} \boldsymbol{v}=\mathbf{0}, \alpha \geq\left\|T_{j} \boldsymbol{\zeta}\right\|(j=1, \ldots, \ell)\right\} .
$$

Then $t^{*}=r_{\min }$ holds for $r_{\min }$ defined in $(20)$, i.e. $t^{*}$ corresponds to the exact minimum value of $\left|u_{q}\right| \cdot$

The proof appears in appendix A.2 in order to improve the readability.

It should be emphasized that the problem (21) is an SOCP problem [2] in the variables $t \in \mathbb{R}$, $\boldsymbol{v} \in \mathbb{R}^{2 d}$, and $\boldsymbol{\zeta} \in \mathbb{R}^{k}$. Hence, we can compute the global optimal solution of (21) easily by using the primal-dual interior-point method.

Remark 3.8. In a manner similar to Proposition 3.7, we can show that $r_{\max }$ is equal to the optimal value of the following problem:

$$
\left.\begin{array}{ll}
\max _{t, \boldsymbol{v}, \boldsymbol{\zeta}} & t \\
\text { s.t. } & t \leq\|G \boldsymbol{v}\|, \\
& S_{1} \boldsymbol{v}=\tilde{\boldsymbol{f}}+F_{0} \boldsymbol{\zeta} \\
& S_{2} \boldsymbol{v}=\mathbf{0}, \\
& \alpha \geq\left\|T_{j} \boldsymbol{\zeta}\right\|, \quad j=1, \ldots, \ell .
\end{array}\right\}
$$

In contrast to (21), the problem (22) is a nonconvex optimization because the constraint condition $t \leq\|G \boldsymbol{v}\|$ is nonconvex. Consequently, standard nonlinear programming approaches are likely to fail for finding the global optimal solution of (22): they converge to a local solution in general. At least, standard nonlinear programming approaches cannot give a proof of the global optimality if they are applied to (22). Certainly the objective value of (22) at a local solution, that is not 


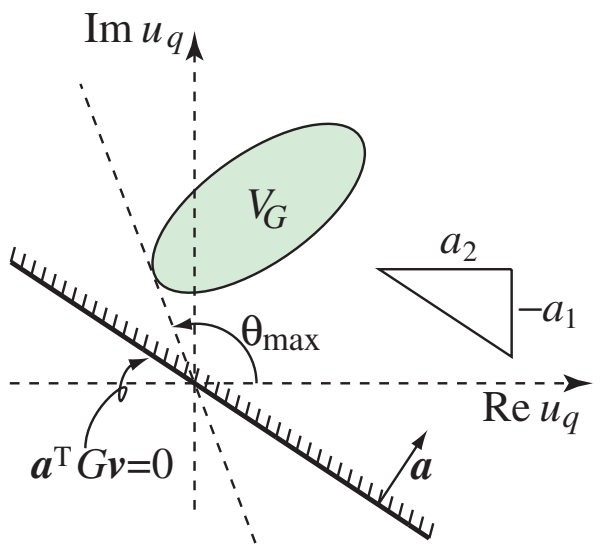

Figure 4: A bounding line which provides an upper bound of $\operatorname{Arg} u_{q}$.

globally optimal, is smaller than $r_{\max }$. It should be emphasized that, for the purpose of robustness analysis, the proof of global optimality is strongly desired, because it guarantees that the modulus of amplitude cannot be larger than the computed value. This motivates us to solve the convex optimization problem (19): its global optimal solution can be computed easily by using an existing algorithm, i.e. the primal-dual interior-point method, and it is guaranteed that $\left|u_{q}\right|$ cannot be larger than the computed value of $\sqrt{\rho^{*}}$.

Note again that by Proposition 3.7 we can compute the exact minimum value of $\left|u_{q}\right|$ efficiently, in contrast with the difficulty of computing the exact maximum value of $\left|u_{q}\right|$ discussed in Remark 3.8.

\subsection{Bounds for phase angle}

In this section, we consider the distribution of the phase angle of $u_{q}(q=1, \ldots, d)$.

In a manner similar to $\left|u_{q}\right|$, we define the maximum value, $\theta_{\max }$, and the minimum value, $\theta_{\min }$, of the phase angle of $u_{q}$ by

$$
\begin{aligned}
\theta_{\max } & :=\max _{\boldsymbol{f}}\left\{\operatorname{Arg} u_{q}(\boldsymbol{f}): \boldsymbol{f} \in \mathcal{F}\right\}, \\
\theta_{\min } & :=\min _{\boldsymbol{f}}\left\{\operatorname{Arg} u_{q}(\boldsymbol{f}): \boldsymbol{f} \in \mathcal{F}\right\} .
\end{aligned}
$$

Throughout this section we assume that $-\pi<\theta_{\min } \leq \theta_{\max }<\pi$.

Remark 3.9. The conditions $-\pi<\theta_{\min }$ and $\theta_{\max }<\pi$ are not always satisfied. For example, if $r_{\text {min }}=0$, then $\theta_{\max }=\pi, \inf _{\boldsymbol{f}}\left\{\operatorname{Arg} u_{q}(\boldsymbol{f}): \boldsymbol{f} \in \mathcal{F}(\alpha)\right\}=-\pi$, and $\theta_{\text {min }}$ is not defined consistently in (24). Such a case can be detected by solving (21), because it provides the exact value of $r_{\min }$; see, e.g., Figure 18 (i) in section 5.2 for an example of $r_{\min }=0$.

We first consider the case in which $0<\theta_{\min } \leq \theta_{\max }<\pi$ is satisfied. The results for the other cases are summarized in Propositions 3.15 and 3.16 below.

In a manner similar to section 3.1, we reformulate the optimization problem (23) into a form of the robust optimization [7]. A key observation is stated as follows: if $u_{q} \in \mathbb{C}$ and $\boldsymbol{a}=\left(a_{i}\right) \in \mathbb{R}^{2}$ 
satisfy

$$
\left[\begin{array}{ll}
a_{1} & a_{2}
\end{array}\right]\left[\begin{array}{l}
\operatorname{Re} u_{q} \\
\operatorname{Im} u_{q}
\end{array}\right]=0
$$

then we obtain

$$
\tan \operatorname{Arg} u_{q}=\frac{\operatorname{Im} u_{q}}{\operatorname{Re} u_{q}}=-\frac{a_{1}}{a_{2}} .
$$

Here, we assume $\operatorname{Re} u_{q} \neq 0$ and $a_{2} \neq 0$ for simplicity. Consequently, it is sufficient to find a bound for $\boldsymbol{a}$ satisfying (25), instead of a bound for $\operatorname{Arg} u_{q}$.

In order to simplify the notation, let $\boldsymbol{e}_{1}, \boldsymbol{e}_{2} \in \mathbb{R}^{2}$ denote

$$
\boldsymbol{e}_{1}=\left[\begin{array}{l}
1 \\
0
\end{array}\right], \quad \boldsymbol{e}_{2}=\left[\begin{array}{l}
0 \\
1
\end{array}\right] .
$$

Proposition 3.10. Assume $0<\theta_{\max }<\pi$. Then $\theta_{\max }$ defined in (23) satisfies

$$
-\frac{1}{\tan \theta_{\max }}=\min _{\boldsymbol{a}}\left\{\boldsymbol{e}_{2}^{\mathrm{T}} \boldsymbol{a}: \boldsymbol{a}^{\mathrm{T}} G \boldsymbol{v} \geq 0(\forall \boldsymbol{v} \in \mathcal{V}), \boldsymbol{e}_{1}^{\mathrm{T}} \boldsymbol{a}=1\right\}
$$

Note that all proofs in this section are collected in appendix A.3 for readability.

A geometrical interpretation of the problem (26) is given in Figure 4. Recall that $\mathcal{V}_{G}=\{G \boldsymbol{v} \mid \boldsymbol{v} \in$ $\mathcal{V}\}$ and $G \boldsymbol{v}=\left(\operatorname{Re} u_{q}, \operatorname{Im} u_{q}\right)^{\mathrm{T}}$. It is observed from Figure 4 that any $\boldsymbol{v}_{G} \in \mathcal{V}_{G}$ satisfies $\boldsymbol{a}^{\mathrm{T}} \boldsymbol{v}_{G} \geq 0$, or, equivalently, $\boldsymbol{v} \in \mathcal{V}$ satisfies $\boldsymbol{a}^{\mathrm{T}} G \boldsymbol{v} \geq 0$. Thus, the normal vector, $\boldsymbol{a}$, of the line in Figure 4 satisfies the constraint condition of the problem (26). It is also observed from Figure 4 that $-a_{1} / a_{2}$, the incline of the line, satisfies $-a_{1} / a_{2} \geq \tan \theta_{\max }$, and hence it gives an upper bound of $\theta_{\max }$. Since the normal vector $\boldsymbol{a}$ is normalized by $\boldsymbol{e}_{1}^{\mathrm{T}} \boldsymbol{a}=a_{1}=1$ in the problem (26), the upper bound mentioned above becomes more tight by minimizing $a_{2}$.

The following proposition gives a sufficient condition for the constraint condition of (26).

Proposition 3.11. The condition

$$
\forall \boldsymbol{v} \in \mathcal{V}: \quad \boldsymbol{a}^{\mathrm{T}} G \boldsymbol{v} \geq 0
$$

holds if there exists a vector $(\boldsymbol{w}, s) \in \mathbb{R}_{+}^{\ell} \times \mathbb{R}$ satisfying

$$
\left[\begin{array}{cc}
O & G^{\mathrm{T}} \boldsymbol{a} \\
\boldsymbol{a}^{\mathrm{T}} G & 0
\end{array}\right] \succeq \sum_{j=1}^{\ell} w_{j} \Psi_{j}+s \Theta .
$$

Based on the sufficient condition provided by Proposition 3.11, we formulate the following problem, which is a robust counterpart of (26), and gives a conservative bound for $\theta_{\max }$.

Proposition 3.12. Assume $0<\theta_{\max }<\pi$. Define $\varphi^{*}$ by

$$
\begin{aligned}
& a_{2}^{*}=\min _{\boldsymbol{a}, \boldsymbol{w}, s}\left\{\boldsymbol{e}_{2}^{\mathrm{T}} \boldsymbol{a}:\left[\begin{array}{cc}
O & G^{\mathrm{T}} \boldsymbol{a} \\
\boldsymbol{a}^{\mathrm{T}} G & 0
\end{array}\right]-\sum_{j=1}^{\ell} w_{j} \Psi_{j}-s \Theta \succeq O, \boldsymbol{w} \geq \mathbf{0}, \boldsymbol{e}_{1}^{\mathrm{T}} \boldsymbol{a}=1\right\}, \\
& \varphi^{*}= \begin{cases}\operatorname{Arctan}\left(-1 / a_{2}^{*}\right), & \left(a_{2}^{*} \leq 0\right), \\
\operatorname{Arctan}\left(-1 / a_{2}^{*}\right)+\pi, & \left(a_{2}^{*}>0\right) .\end{cases}
\end{aligned}
$$

Then $\theta_{\max } \leq \varphi^{*}$ holds for $\theta_{\max }$ defined in (23), i.e. $\varphi^{*}$ corresponds to an upper bound for $\operatorname{Arg} u_{q}$. 


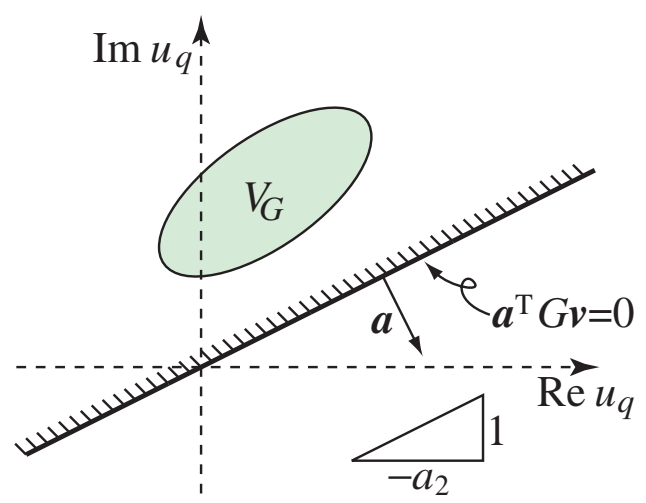

Figure 5: A bounding line which provides a lower bound of $\operatorname{Arg} u_{q}$.

The proof appears in appendix A.3.3.

Note that (29) is an SDP problem in the variables $\boldsymbol{a} \in \mathbb{R}^{2}, \boldsymbol{w} \in \mathbb{R}^{\ell}$, and $s \in \mathbb{R}$, and hence it can be solved easily.

We next consider a lower bound for $\theta_{\text {min }}$. Analogous to Proposition 3.10, the definition, (24), of $\theta_{\min }$ is rewritten into the form of robust optimization as

$$
-\frac{1}{\tan \theta_{\min }}=\max _{\boldsymbol{a}}\left\{\boldsymbol{e}_{2}^{\mathrm{T}} \boldsymbol{a}: \boldsymbol{a}^{\mathrm{T}} G \boldsymbol{v} \leq 0(\forall \boldsymbol{v} \in \mathcal{V}), \boldsymbol{e}_{1}^{\mathrm{T}} \boldsymbol{a}=1\right\} .
$$

Figure 5 shows an example of $\boldsymbol{a}$ satisfying the constraint condition $\boldsymbol{a}^{\mathrm{T}} G \boldsymbol{v} \leq 0(\forall \boldsymbol{v} \in \mathcal{V})$ in (31). A sufficient condition for this constraint condition is given in the following proposition.

Proposition 3.13. The condition

$$
\forall \boldsymbol{v} \in \mathcal{V}: \quad \boldsymbol{a}^{\mathrm{T}} G \boldsymbol{v} \leq 0
$$

holds if there exists a vector $(\boldsymbol{w}, s) \in \mathbb{R}_{+}^{\ell} \times \mathbb{R}$ satisfying

$$
\left[\begin{array}{cc}
O & -G^{\mathrm{T}} \boldsymbol{a} \\
-\boldsymbol{a}^{\mathrm{T}} G & 0
\end{array}\right] \succeq \sum_{j=1}^{\ell} w_{j} \Psi_{j}+s \Theta .
$$

The proof is analogous to Proposition 3.11, and hence is omitted.

We construct a robust counterpart to the problem (31) by replacing the constraint condition $\boldsymbol{a}^{\mathrm{T}} G \boldsymbol{v} \leq 0(\forall \boldsymbol{v} \in \mathcal{V})$ with its sufficient condition provided by Proposition 3.13. Thus we obtain an SDP problem which gives a lower bound of $\operatorname{Arg} u_{q}$ as follows.

Proposition 3.14. Assume $0<\theta_{\min }<\pi$. Put $a_{2}^{*}$ as

$$
a_{2}^{*}=\max _{\boldsymbol{a}, \boldsymbol{w}, s}\left\{\boldsymbol{e}_{2}^{\mathrm{T}} \boldsymbol{a}:\left[\begin{array}{cc}
O & -G^{\mathrm{T}} \boldsymbol{a} \\
-\boldsymbol{a}^{\mathrm{T}} G & 0
\end{array}\right]-\sum_{j=1}^{\ell} w_{j} \Psi_{j}-s \Theta \succeq O, \boldsymbol{w} \geq \mathbf{0}, \boldsymbol{e}_{1}^{\mathrm{T}} \boldsymbol{a}=1\right\},
$$

and define $\varphi^{*}$ by (30). Then $\varphi^{*} \leq \theta_{\min }$ holds for $\theta_{\min }$ defined in (24), i.e. $\varphi^{*}$ corresponds to a lower bound for $\operatorname{Arg} u_{q}$.

The proof is analogous to Proposition 3.12, and hence is omitted.

For the cases of $-\pi<\theta_{\max }<0$ and $-\pi<\theta_{\min }<0$, we show only results in the following two propositions. 
Proposition 3.15. Assume $-\pi<\theta_{\max }<0$. Define $\varphi^{*}$ by

$$
\begin{aligned}
& a_{2}^{*}=\min _{\boldsymbol{a}, \boldsymbol{w}, s}\left\{\boldsymbol{e}_{2}^{\mathrm{T}} \boldsymbol{a}:\left[\begin{array}{cc}
O & -G^{\mathrm{T}} \boldsymbol{a} \\
-\boldsymbol{a}^{\mathrm{T}} G & 0
\end{array}\right]-\sum_{j=1}^{\ell} w_{j} \Psi_{j}-s \Theta \succeq O, \boldsymbol{e}_{1}^{\mathrm{T}} \boldsymbol{a}=1, \boldsymbol{w} \geq \mathbf{0}, \boldsymbol{e}_{1}^{\mathrm{T}} \boldsymbol{a}=1\right\}, \\
& \varphi^{*}= \begin{cases}\operatorname{Arctan}\left(-1 / a_{2}^{*}\right), & \left(a_{2}^{*} \leq 0\right), \\
\operatorname{Arctan}\left(-1 / a_{2}^{*}\right)-\pi, & \left(a_{2}^{*}>0\right) .\end{cases}
\end{aligned}
$$

Then $\theta_{\max } \leq \varphi^{*}$ holds for $\theta_{\max }$ defined in (23), i.e. $\varphi^{*}$ corresponds to an upper bound for $\operatorname{Arg} u_{q}$.

Proposition 3.16. Assume $-\pi<\theta_{\min }<0$. Put $a_{2}^{*}$ as

$$
a_{2}^{*}=\max _{\boldsymbol{a}, \boldsymbol{w}, s}\left\{\boldsymbol{e}_{2}^{\mathrm{T}} \boldsymbol{a}:\left[\begin{array}{cc}
O & G^{\mathrm{T}} \boldsymbol{a} \\
\boldsymbol{a}^{\mathrm{T}} G & 0
\end{array}\right]-\sum_{j=1}^{\ell} w_{j} \Psi_{j}-s \Theta \succeq O, \boldsymbol{e}_{1}^{\mathrm{T}} \boldsymbol{a}=1, \boldsymbol{w} \geq \mathbf{0}, \boldsymbol{e}_{1}^{\mathrm{T}} \boldsymbol{a}=1\right\},
$$

and define $\varphi^{*}(34)$. Then $\varphi^{*} \leq \theta_{\min }$ holds for $\theta_{\min }$ defined in (24), i.e. $\varphi^{*}$ corresponds to a lower bound for $\operatorname{Arg} u_{q}$.

Consequently, an upper bound for $\operatorname{Arg} u_{q}$ can be obtained by solving either (29) or (33); a lower bound for $\operatorname{Arg} u_{q}$ can be obtained by solving either (32) or (35).

Remark 3.17. In this section 3 we have investigated the distribution of the amplitude of the displacement, $u_{q}$. For this purpose we define the constant matrix $G$ by (13). Certainly the SDP and SOCP formulations presented are irrespective to the definition of $G$, which implies that those formulations are valid for computing bounds for any linear function of the displacement vector. For example, the member stress of a truss is written as a linear function of the displacement vector, and hence we can compute bounds for the amplitude of member stress by using the formulations presented in section 3; see section 5.1.3 for numerical examples of bounds for member stresses.

\subsection{Comparison with ellipsoidal bound method for static analysis}

In [22] we proposed an SDP formulation to find a confidential ellipsoidal bound for static response of an uncertain structural system. It is absolutely clear that static problems and dynamic problems are completely different in view of governing equations and state variables (real variables in static problems and complex variables in dynamic problems). Because we restrict ourselves to the steadystate caused by a forced oscillation, it may be possible to apply the method presented in [22] to find an ellipsoidal bound for the distribution of $G \boldsymbol{v}$. However, such an ellipsoidal bound cannot be utilized directly to obtain a conservative bound for $\left|u_{q}\right|$ and $\operatorname{Arg} u_{q}$. Hence, we have presented a new approach to the dynamic problem in sections 3.1-3.3. In this section we give in-depth comparison of the methods presented in this paper and in [22].

\subsubsection{Finding upper bound for modulus}

In section 3.1 we have presented a method for finding an upper bound for $\left|u_{q}\right|$. It is interesting to see that the problem (7) is equivalent to finding the minimal circle which is centered at the origin and includes all possible realization of $G \boldsymbol{v}$, where $\boldsymbol{v}$ solves (10) and (11). 
On the other hand, in [22] we proposed a method to find an ellipsoidal bound for the static response of an uncertain structural system. Since finding a circular bound is regarded as a particular case of finding an ellipsoidal bound, we can apply the method in [22] directly for finding an upper bound for $\left|u_{q}\right|$, which results in the SDP problem

$$
\bar{\rho}^{*}=\min _{\rho, \boldsymbol{w}, s}\left\{\rho:\left[\begin{array}{c|c}
\rho I_{2} & {\left[\begin{array}{ll}
G & \mathbf{0}
\end{array}\right]} \\
\hline\left[\begin{array}{l}
G^{\mathrm{T}} \\
\mathbf{0}^{\mathrm{T}}
\end{array}\right] & \operatorname{diag}(\mathbf{0}, 1)-\sum_{j=1}^{\ell} w_{j} \Psi_{j}-s \Theta
\end{array}\right] \succeq O, \boldsymbol{w} \geq \mathbf{0}\right\},
$$

where $\rho, \boldsymbol{w}$, and $s$ are the variables. Then it is guaranteed that $\sqrt{\bar{\rho}^{*}}$ corresponds to an upper bound of $\left|u_{q}\right|$.

Note that the problem (36) includes a constraint condition such that a $(2 d+3) \times(2 d+3)$ matrix should be positive semidefinite, while in the problem $(19)$ a $(2 d+1) \times(2 d+1)$ matrix is required to be positive semidefinite. Thus, in section 3.1 we have presented the SDP problem with the smaller size than the formulation in [22] which was proposed for a general ellipsoidal bound.

\subsubsection{Finding lower bound for modulus}

In section 3.2 we have presented a method for finding the minimum value of $\left|u_{q}\right|$. Note that finding the minimum value $\left|u_{q}\right|$ is equivalent to finding the maximal circle which is centered at the origin and does not includes all possible realization of $G \boldsymbol{v}$. Hence, in contrast to the case of section 3.4.1, it is difficult to apply the method presented in [22] directly to finding a lower bound for $\left|u_{q}\right|$.

Moreover, Proposition 3.7 shows that we can compute the exact minimum value of $\left|u_{q}\right|$ from (21), which can be solved efficiently by using the primal-dual interior-point method. Hence, it is clear that the approach presented in section 3.2 is more suitable for the dynamic steady-state analysis including uncertainties compared with any conservative method, e.g. [22], which does not have a proof of exactness.

\subsubsection{Finding bounds for phase angles}

In section 3.3 we have presented a method for finding upper and lower bounds for the phase angle of $u_{q}$. It is noted that the objective function, $\operatorname{Arg} u_{q}=\operatorname{Im} u_{q} / \operatorname{Re} u_{q}$, of the problems (23) and (24) is a nonlinear function of the vector $\left(\operatorname{Re} u_{q}, \operatorname{Im} u_{q}\right)^{\mathrm{T}}=G \boldsymbol{v}$.

In [22] we have restricted ourselves to finding an ellipsoidal bound for a linear function of the displacements of static problems. In contrast, we aim at finding bounds for the nonlinear function $\operatorname{Arg} u_{q}$ of $(\operatorname{Re} \boldsymbol{u}, \operatorname{Im} \boldsymbol{u})$ in the problems (23) and (24), and hence it is not possible to apply the approach in [22] to those problems.

\subsubsection{Comparison with two-phase method using ellipsoidal bound}

As discussed in sections 3.4.2 and 3.4.3, it is difficult to apply the method in [22] directly to finding a lower bound for $\left|u_{q}\right|$ and bounds for $\operatorname{Arg} u_{q}$ in the present dynamic steady-state problem. Even in the case of finding an upper bound for $\left|u_{q}\right|$, the method in [22] has a drawback such that the resulting SDP problem has the larger size as shown in section 3.4.1, compared with the approach proposed in section 3.1. 
There may remain a possibility of developing a 'two-phase method' in which we firstly compute an ellipsoidal bound by using the formulation in [22] and secondly compute bounds for $\left|u_{q}\right|$ and $\operatorname{Arg} u_{q}$ from the obtained ellipsoid. We next show that such a two-phase method has serious drawbacks compared with a direct method proposed in this paper. We compare such a two-phase approach and the method developed in this paper from the two points of view. The one is the tractability of the optimization problems which are to be solved (section 3.4.4 (a)), and the other the tightness of the obtained bounds (section 3.4.4 (b)). The insufficiency of the two-phase method is shown in the following manner:

(i) Finding an ellipsoidal bound for $\left(\operatorname{Re} u_{q}, \operatorname{Im} u_{q}\right)$ by applying the method in [22];

(ii) Finding conservative bounds for $\left|u_{q}\right|$ and $\operatorname{Arg} u_{q}$ by using the results of (i);

(iii) Showing the reason why the bounds obtained in (ii) are insufficient.

Since $\left|u_{q}\right|$ and $\operatorname{Arg} u_{q}$ are nonlinear functions of $(\operatorname{Re} u, \operatorname{Im} u)$, it is difficult to perform the step (ii) in fact. In the following we consider two alternative approaches to the step (ii): the one is based on the nonlinear programming (section 3.4.4 (a)), and the other is a heuristic approach based on the random sampling method (section 3.4.4 (b)).

(a) Tractability of optimization problems We here discuss the difficulty of the optimization problems to be solved at the step (ii).

(a)-(i) Since $G \boldsymbol{v} \in \mathbb{R}^{2}$ in (13) is a real vector, we can use the method presented in [22] to obtain an ellipsoidal bound for all possible $G \boldsymbol{v}$. More precisely, we can compute $P \in \mathcal{S}^{2}$ and $\hat{\boldsymbol{z}} \in \mathbb{R}^{2}$ such that the ellipsoid defined by

$$
\mathcal{E}=\left\{\boldsymbol{z} \in \mathbb{R}^{2} \mid\left[\begin{array}{cc}
P & (\boldsymbol{z}-\hat{\boldsymbol{z}}) \\
(\boldsymbol{z}-\hat{\boldsymbol{z}})^{\mathrm{T}} & 1
\end{array}\right] \succeq O\right\}
$$

satisfies $G \boldsymbol{v} \in \mathcal{E}_{x}$ for any $\boldsymbol{v}$ by solving (10) and (11).

As an illustrative example we compute ellipsoidal bounds for $\left(\operatorname{Re} u_{x}, \operatorname{Im} u_{x}\right)$ and $\left(\operatorname{Re} u_{y}, \operatorname{Im} u_{y}\right)$ of the truss shown in Figure 6. See section 5.1.1 for the details of this truss example. For the displacement amplitude in the $x$-direction, $\left(\operatorname{Re} u_{x}, \operatorname{Im} u_{x}\right)$, the parameters of the bounding ellipsoid in (37) are computed as

$$
P=\left[\begin{array}{cc}
2.2357 \times 10^{-4} & -9.8146 \times 10^{-6} \\
-9.8146 \times 10^{-6} & 0.251257
\end{array}\right], \quad \hat{z}=\left[\begin{array}{c}
0.140893 \\
-1.158237
\end{array}\right]
$$

by using the method in [22]. Similarly, for $\left(\operatorname{Re} u_{y}, \operatorname{Im} u_{y}\right)$ we obtain

$$
P=\left[\begin{array}{cc}
2.4544 \times 10^{-4} & -5.4593 \times 10^{-7} \\
-5.4593 \times 10^{-7} & 4.508587
\end{array}\right], \quad \hat{z}=\left[\begin{array}{l}
0.033260 \\
4.876861
\end{array}\right] .
$$


(a)-(ii) Since the bound $\mathcal{E}$ in (37) is conservative, the optimal values of the following optimization problems correspond to conservative bounds for $r_{\max }$ and $r_{\min }$ :

$$
\begin{array}{ll}
\max _{\boldsymbol{z}}\{\|\boldsymbol{z}\| \mid \boldsymbol{z} \in \mathcal{E}\} & \left(\geq r_{\max }\right), \\
\min _{\boldsymbol{z}}\{\|\boldsymbol{z}\| \mid \boldsymbol{z} \in \mathcal{E}\} & \left(\leq r_{\min }\right) .
\end{array}
$$

Similarly, conservative bounds for $\theta_{\max }$ and $\theta_{\min }$ are obtained as the optimal values of the following optimization problems:

$$
\begin{array}{cl}
\max _{\boldsymbol{z}}\left\{z_{2} / z_{1} \mid \boldsymbol{z} \in \mathcal{E}_{x}\right\} & \left(\geq \theta_{\max }\right), \\
\min _{\boldsymbol{z}}\left\{z_{2} / z_{1} \mid \boldsymbol{z} \in \mathcal{E}_{x}\right\} & \left(\leq \theta_{\min }\right) .
\end{array}
$$

A crucial point of the two-phase approach is the solvability of the nonlinear programming problems (38)-(41). Since $\mathcal{E}$ defined in (37) is a convex set, we see that (39) is a convex optimization problem. Hence, it is easy to obtain a lower bound of $r_{\min }$ by solving (39).

In contrast to (39), it is emphasized that (38), (40), and (41) are nonconvex problems. Hence, it is difficult to find the global optimal values of (38), (40), and (41). Note that conventional nonlinear programming methods are not acceptable, because they do not give a proof of the global optimality for those nonconvex problems and hence we lose a guarantee that the obtained bound is conservative. This is a crucial drawback of a two-phase approach based on the method in [22].

(a)-(iii) As discussed in section 3.4.4 (a)-(ii), we can solve the problem (39) by using a conventional nonlinear programming in order to find a lower bound of $r_{\min }$. Note that such a lower bound is not equal to $r_{\text {min }}$ in general, because it is not guaranteed that the ellipsoidal bound $\mathcal{E}$ found by the method in [22] corresponds to the exact bound. In contrast, as mentioned in section 3.4.2, in this paper we have presented a formulation to find the exact value of $r_{\min }$, which is certainly preferred to a conservative bound found in (a)-(ii). We shall revisit the tightness issue of bounds for $r_{\min }$ in section 3.4.4 (b)-(iii) below.

Concerning bounds for $r_{\max }, \theta_{\max }$, and $\theta_{\min }$, there exists no efficient algorithm for computing the global optimal solutions of the problems (38), (40), and (41), which are nonconvex optimization problems. This is the reason why we have developed new formulations for dynamic steady-state problem in this paper.

In addition, even if an efficient algorithm for finding a conservative solution for (a)-(ii) is available, the obtained result may not be better than the one obtained by using a direct method presented below. This is because we may lose tightness of the bound in general at each phase of a multi-phase method. Consequently, independently of [22] we have presented a new approach which deals with the problems (7), (20), (23), and (24) directly.

(b) Tightness of bounds We here compare the bounds obtained by using a two-phase method and the proposed method in order to show that the two-phase method is not sufficient from the viewpoint of tightness of the bounds. We again consider the truss example shown in Figure 6, where the details are described in section 5.1.1. 
Table 1: Comparison of bounds obtained by a two-phase method and bounds shown in Figure 7 which are obtained by the proposed method.

\begin{tabular}{ccc}
\hline & two-phase method & proposed method \\
\hline$\left|u_{x}\right|(\mathrm{cm})$ & $0.671906 \leq\left|u_{x}\right| \leq 1.665465$ & $0.671910 \leq\left|u_{x}\right| \leq 1.665462$ \\
$\left|u_{y}\right|(\mathrm{cm})$ & $2.753718 \leq\left|u_{y}\right| \leq 7.000283$ & $2.753729 \leq\left|u_{y}\right| \leq 7.000272$ \\
\hline $\operatorname{Arg} u_{x}(\mathrm{rad})$ & $-1.487640 \leq \operatorname{Arg} u_{x} \leq-1.358074$ & $-1.487551 \leq \operatorname{Arg} u_{x} \leq-1.358148$ \\
$\operatorname{Arg} u_{y}(\mathrm{rad})$ & $1.557267 \leq \operatorname{Arg} u_{y} \leq 1.567496$ & $1.558616 \leq \operatorname{Arg} u_{y} \leq 1.566157$ \\
\hline
\end{tabular}

(b)-(i) By using the method in [22], ellipsoidal bounds can be obtained for $\left(\operatorname{Re} u_{x}, \operatorname{Im} u_{x}\right)$ and $\left(\operatorname{Re} u_{y}, \operatorname{Im} u_{y}\right)$ as shown in section 3.4.4 (a)-(i).

(b)-(ii) As mentioned in section 3.4.4 (a)-(ii), it is very difficult to solve the problems (38)-(41). In order to estimate the optimal solutions of (38)-(41), we randomly generate a number of sample points on the boundary of $\mathcal{E}$ in (37) in order to find approximate solutions to (38)-(41). The obtained results are listed in Table 1. Note that such a procedure based on the random sampling is not acceptable from the theoretical point of view, i.e. we can examine only finite number of sample points, which implies that the obtained results underestimate the optimal values of (38)-(41) and we lose a proof of the conservativeness. In addition it is not straightforward to generate uniformly distributing samples for our problem in section 4, where we consider uncertainty sets in the four- or six-dimensional space.

(b)-(iii) The bounds obtained by using the method presented in this paper are also listed in Table 1. It is observed in Table 1 that the solutions obtained by random sampling from the ellipsoidal bounds of [22] overestimate $\left|u_{q}\right|$ and $\operatorname{Arg} u_{q}$ even for such a small structural system. Because random sampling procedure underestimates the solutions to (38)-(41), this result implies that the exact optimal values of (38)-(41) are overestimated bounds compared with the method proposed in this paper.

Summary of comparison In sections 3.4.4 (a) and (b), it has been shown that, both in the qualitative comparison and in the quantitative one, the method in [22] is not sufficient for finding tight bounds for $\left|u_{q}\right|$ and $\operatorname{Arg} u_{q}$. This result may be intuitively interpreted as follows. By the method in [22] we first compute the ellipsoidal bound in (37), and secondly try to compute bounds which we actually want. In contrast, by the proposed method we directly compute four bounds as illustrated in Figure 7. It may be natural that a bound obtained directly is tighter than a bound obtained by using a two-phase method. Thus, the approach presented in this paper is truly desired for the dynamic steady-state analysis under uncertain harmonic loads. 


\section{Bounds for nodal oscillation}

Consider a structure in the two-dimensional space. For the $p$ th node we denote by $u_{p x} \in \mathbb{C}$ and $u_{p y} \in$ $\mathbb{C}$ the nodal displacement in the $x$ - and $y$-th directions, respectively. We have investigated bounds for $\left|u_{p x}\right|$ and $\operatorname{Arg} u_{p x}$ in section 3. In this section we consider the distribution of $\boldsymbol{\nu}_{p}=\left(\nu_{p x}, \nu_{p y}\right) \in \mathbb{R}^{2}$ defined by

$$
\boldsymbol{\nu}_{p}=\left[\begin{array}{l}
\left|u_{p x}\right| \\
\left|u_{p y}\right|
\end{array}\right] .
$$

More precisely, we propose optimization problems in order to find bounds for $\left\|\boldsymbol{\nu}_{p}\right\|$ and $\nu_{p y} / \nu_{p x}$.

Remark 4.1. In this section we consider bounds for $\sqrt{\left|u_{p x}\right|^{2}+\left|u_{p y}\right|^{2}}$ and $\left|u_{p y}\right| /\left|u_{p x}\right|$ in order to estimate the distribution of $\boldsymbol{\nu}_{p}=\left(\left|u_{p x}\right|,\left|u_{p y}\right|\right)$. Note that further information of the distribution has been obtained from the results in sections 3.1 and 3.2, where we have investigated upper and lower bounds for $\left|u_{q}\right|$. Consequently, we can compute upper and lower bounds for $\sqrt{\left|u_{p x}\right|^{2}+\left|u_{p y}\right|^{2}}$, $\left|u_{p y}\right| /\left|u_{p x}\right|,\left|u_{p x}\right|$, and $\left|u_{p y}\right|$. The vector $\left(\left|u_{p x}\right|,\left|u_{p y}\right|\right)$ can exist in the intersection of those bounds; see Figure 11 in section 5.1.2 for illustrative examples.

\subsection{Bounds for norm of moduli}

In this section we consider bounds for $\left\|\boldsymbol{\nu}_{p}\right\|$. Recall that $\boldsymbol{\nu}_{p}$ depends on $\boldsymbol{f}$. We denote by $r_{\max }^{\nu}$ and $r_{\min }^{\nu}$ the maximum and minimum values of $\left\|\boldsymbol{\nu}_{p}\right\|$, which are defined by

$$
\begin{aligned}
& r_{\text {max }}^{\nu}=\max _{\boldsymbol{f}}\left\{\left\|\boldsymbol{\nu}_{p}(\boldsymbol{f})\right\|: \boldsymbol{f} \in \mathcal{F}\right\}, \\
& r_{\text {min }}^{\nu}=\min _{\boldsymbol{f}}\left\{\left\|\boldsymbol{\nu}_{p}(\boldsymbol{f})\right\|: \boldsymbol{f} \in \mathcal{F}\right\} .
\end{aligned}
$$

We can obtain bounds for $\left\|\boldsymbol{\nu}_{p}\right\|$ in similar manners to sections 3.1 and 3.2. From the definition of $\boldsymbol{\nu}_{p}$, we obtain

$$
\left\|\boldsymbol{\nu}_{p}\right\|=\left\|\left[\begin{array}{c}
\left|u_{p x}\right| \\
\left|u_{p y}\right|
\end{array}\right]\right\|=\left\|\left[\begin{array}{c}
\operatorname{Re} u_{p x} \\
\operatorname{Im} u_{p x} \\
\operatorname{Re} u_{p y} \\
\operatorname{Im} u_{p y}
\end{array}\right]\right\| .
$$

Let $\hat{G} \in \mathbb{R}^{4 \times 2 d}$ denote a constant matrix satisfying

$$
\hat{G} \boldsymbol{v}=\left[\begin{array}{c}
\operatorname{Re} u_{p x} \\
\operatorname{Im} u_{p x} \\
\operatorname{Re} u_{p y} \\
\operatorname{Im} u_{p y}
\end{array}\right]
$$

Then the problem (42) is reduced to

$$
r_{\max }^{\nu}=\max _{\boldsymbol{v}}\{\|\hat{G} \boldsymbol{v}\|: \boldsymbol{v} \in \mathcal{V}\}
$$

which is mathematically equivalent to the problem investigated in section 3.1. Consequently, we obtain an SDP problem giving an upper bound for $r_{\max }^{\nu}$ from (19) in Proposition 3.6, by replacing $G$ with $\hat{G}$. Similarly, an SOCP problem for computing $r_{\min }^{\nu}$ can be obtained from (21) in Proposition 3.7 by replacing $G$ with $\hat{G}$. These observations are summarized in the following two propositions. 
Proposition 4.2. Define $\rho^{*}$ by

$$
\rho^{*}=\min _{\rho, \boldsymbol{w}, s}\left\{\rho:\left[\begin{array}{cc}
-\hat{G}^{\mathrm{T}} \hat{G} & \mathbf{0} \\
\mathbf{0}^{\mathrm{T}} & \rho
\end{array}\right]-\sum_{j=1}^{\ell} w_{j} \Psi_{j}-s \Theta \succeq O, \boldsymbol{w} \geq \mathbf{0}\right\} .
$$

Then $\left(r_{\max }^{\nu}\right)^{2} \leq \rho^{*}$ holds for $r_{\max }^{\nu}$ defined in $(42)$, i.e. $\sqrt{\rho^{*}}$ corresponds to an upper bound of $\left\|\boldsymbol{\nu}_{p}\right\|$.

Proposition 4.3. $r_{\min }^{\nu}$ defined in (43) satisfies

$$
t^{*}=\min _{t, \boldsymbol{v}, \boldsymbol{\zeta}}\left\{t: r \geq\|\hat{G} \boldsymbol{v}\|, S_{1} \boldsymbol{v}=\tilde{\boldsymbol{f}}+F_{0} \boldsymbol{\zeta}, S_{2} \boldsymbol{v}=\mathbf{0}, \alpha \geq\left\|T_{j} \boldsymbol{\zeta}\right\|(j=1, \ldots, \ell)\right\},
$$

i.e. the minimum value of $\left\|\boldsymbol{\nu}_{p}\right\|$ can be obtained by solving the problem (45).

Note that the SDP problem (44) has the smaller size compared with the SDP formulation presented in [22]; see section 3.4.1 for more details. Proposition 4.3 implies that the exact lower bound can be obtained efficiently by solving the SOCP problem (45).

\subsection{Bounds for ratio of moduli}

We consider bounds for ratio $\nu_{p y} / \nu_{p x}$. For this purpose, we attempt to compute bounds for $\nu_{p y}^{2} / \nu_{p x}^{2}$ instead of $\nu_{p y} / \nu_{p x}$. Introduce auxiliary variables $\mu_{x}=\left(\operatorname{Re} u_{p x}\right)^{2}+\left(\operatorname{Im} u_{p x}\right)^{2}=\nu_{p x}^{2}$ and $\mu_{y}=$ $\left(\operatorname{Re} u_{p y}\right)^{2}+\left(\operatorname{Im} u_{p y}\right)^{2}=\nu_{p y}^{2}$, and we consider bounds for $\mu_{y} / \mu_{x}$. Note that $\mu_{y} \geq 0$ and $\mu_{x} \geq 0$. We assume $\mu_{x} \neq 0$.

In a manner similar to section 3.3, we aim at finding bounds for $\mu_{y} / \mu_{x}$, which is a nonlinear function of $(\operatorname{Re} \boldsymbol{u}, \operatorname{Im} \boldsymbol{u})$. This is the major dificulty of the problem dealt with this section, compared with the situation considered in [22] (see section 3.4.3 for more deteils).

The maximum value, $t_{\max }$, of $\left|u_{p y}\right| /\left|u_{p x}\right|$ is defined by

$$
t_{\max }=\max _{\boldsymbol{f}}\left\{\nu_{p y} / \nu_{p x}: \nu_{p x}=\left|u_{p x}(\boldsymbol{f})\right|, \nu_{p y}=\left|u_{p y}(\boldsymbol{f})\right|, \boldsymbol{f} \in \mathcal{F}\right\} .
$$

We attempt to find an upper bound for $t_{\max }$.

Define $G_{p x}, G_{p y} \in \mathbb{R}^{2 \times 2 d}$ as constant matrices satisfying

$$
G_{x} \boldsymbol{v}=\left[\begin{array}{c}
\operatorname{Re} u_{p x} \\
\operatorname{Im} u_{p x}
\end{array}\right], \quad G_{y} \boldsymbol{v}=\left[\begin{array}{c}
\operatorname{Re} u_{p y} \\
\operatorname{Im} u_{p y}
\end{array}\right] .
$$

By using $\mathcal{V}$ defined in (12), (46) is reduced to

$$
t_{\max }=\max _{\boldsymbol{v}}\left\{\nu_{y} / \nu_{x}: \nu_{x}=\left\|G_{x} \boldsymbol{v}\right\|, \nu_{y}=\left\|G_{y} \boldsymbol{v}\right\|, \boldsymbol{v} \in \mathcal{V}\right\}
$$

However, instead of solving this problem directly, we treat

$$
\left(t_{\max }\right)^{2}=\max _{\boldsymbol{v}}\left\{\mu_{y} / \mu_{x}: \mu_{x}=\left\|G_{x} \boldsymbol{v}\right\|^{2}, \mu_{y}=\left\|G_{y} \boldsymbol{v}\right\|^{2}, \boldsymbol{v} \in \mathcal{V}\right\}
$$

In a manner similar to Proposition 3.10, we reformulate the problem (48) into the form of robust optimization [7] as follows. Notice here that $\mu_{x} \geq 0$ and $\mu_{y} \geq 0$ are satisfied in (48), which corresponds to the assumption in Proposition 3.10. 
Proposition 4.4. $t_{\max }$ defined in (46) satisfies

$$
-\frac{1}{\left(t_{\max }\right)^{2}}=\min _{\boldsymbol{a}}\left\{\boldsymbol{e}_{2}^{\mathrm{T}} \boldsymbol{a}: \boldsymbol{a}^{\mathrm{T}} \boldsymbol{\mu} \geq 0\left(\forall \boldsymbol{\mu} \in\left\{\left(\left\|G_{x} \boldsymbol{v}\right\|^{2},\left\|G_{y} \boldsymbol{v}\right\|^{2}\right)^{\mathrm{T}} \mid \boldsymbol{v} \in \mathcal{V}\right\}\right), \boldsymbol{e}_{1}^{\mathrm{T}} \boldsymbol{a}=1\right\} .
$$

The proof is analogous to Proposition 3.10, and hence is omitted.

Let $\hat{\Theta}_{1}, \hat{\Theta}_{2} \in \mathcal{S}^{2 d+3}$ be

$$
\hat{\Theta}_{1}=\left[\begin{array}{ccc}
O & O & {\left[\begin{array}{c}
1 / 2 \\
0
\end{array}\right]} \\
O & -G_{p x} G_{p x}^{\mathrm{T}} & \mathbf{0} \\
{\left[\begin{array}{cc}
1 / 2 & 0
\end{array}\right]} & \mathbf{0}^{\mathrm{T}} & 0
\end{array}\right], \quad \hat{\Theta}_{2}=\left[\begin{array}{ccc}
O & O & {\left[\begin{array}{c}
0 \\
1 / 2
\end{array}\right]} \\
O & -G_{p y} G_{p y}^{\mathrm{T}} & \mathbf{0} \\
{\left[\begin{array}{cc}
0 & 1 / 2
\end{array}\right]} & \mathbf{0}^{\mathrm{T}} & 0
\end{array}\right],
$$

By using $\Psi_{j}(j=1, \ldots, \ell)$ and $\Theta$ defined in Proposition 3.4 , define $\hat{\Psi}_{1}, \ldots, \hat{\Psi}_{\ell} \in \mathcal{S}^{2 d+3}$ and $\hat{\Theta}_{3} \in$ $\mathcal{S}^{2 d+3}$ by

$$
\begin{aligned}
\hat{\Psi}_{j} & =\left[\begin{array}{ll}
O & O \\
O & \Psi_{j}
\end{array}\right], \quad j=1, \ldots, \ell, \\
\hat{\Theta}_{3} & =\left[\begin{array}{ll}
O & O \\
O & \Theta
\end{array}\right] .
\end{aligned}
$$

As a conservative approximation of the problem (49), we obtain the following SDP problem which provides an upper bound for $t_{\max }$.

Proposition 4.5. Define $\varphi^{*}$ by

$$
a_{2}^{*}=\min _{\boldsymbol{a}, \boldsymbol{w}, \boldsymbol{s}}\left\{\boldsymbol{e}_{2}^{\mathrm{T}} \boldsymbol{a}:\left[\begin{array}{ccc}
O & O & \boldsymbol{a} \\
O & O & \mathbf{0} \\
\boldsymbol{a}^{\mathrm{T}} & \mathbf{0}^{\mathrm{T}} & 0
\end{array}\right]-\sum_{j=1}^{\ell} w_{j} \hat{\Psi}_{j}-\sum_{i=1}^{3} s_{i} \hat{\Theta}_{i} \succeq O, \boldsymbol{w} \geq \mathbf{0}, \boldsymbol{e}_{1}^{\mathrm{T}} \boldsymbol{a}=1\right\}
$$

and $\varphi^{*}=\sqrt{-1 / a_{2}^{*}}$. Then $t_{\max } \leq \varphi^{*}$ holds for $t_{\max }$ defined in (46), i.e. $\varphi^{*}$ corresponds to an upper bound for $\mu_{y} / \mu_{x}$.

The proof appears in appendix A.4.

We next consider the minimum value, $t_{\min }$, of $\left|u_{p y}\right| /\left|u_{p x}\right|$ which is defined by

$$
t_{\min }=\min _{\boldsymbol{f}}\left\{\nu_{p y} / \nu_{p x}: \nu_{p x}=\left|u_{p x}(\boldsymbol{f})\right|, \nu_{p y}=\left|u_{p y}(\boldsymbol{f})\right|, \boldsymbol{f} \in \mathcal{F}\right\} .
$$

A robust optimization reformulation of (51) is obtained as

$$
-\frac{1}{\left(t_{\min }\right)^{2}}=\max _{\boldsymbol{a}}\left\{\boldsymbol{e}_{2}^{\mathrm{T}} \boldsymbol{a}: \boldsymbol{a}^{\mathrm{T}} \boldsymbol{\mu} \leq 0 \quad\left(\forall \boldsymbol{\mu} \in\left\{\left(\left\|G_{p x} \boldsymbol{v}\right\|^{2},\left\|G_{p y} \boldsymbol{v}\right\|^{2}\right)^{\mathrm{T}} \mid \boldsymbol{v} \in \mathcal{V}\right\}\right), \boldsymbol{e}_{1}^{\mathrm{T}} \boldsymbol{a}=1\right\} .
$$

In a manner similar to Proposition 4.5, we can show that a lower bound for $t_{\min }$ is obtained by solving the following SDP problem.

Proposition 4.6. Define $\varphi^{*}$ by

$$
\max _{\boldsymbol{a}, \boldsymbol{w}, \boldsymbol{s}}\left\{\boldsymbol{e}_{2}^{\mathrm{T}} \boldsymbol{a}:\left[\begin{array}{ccc}
O & O & -\boldsymbol{a} \\
O & O & \mathbf{0} \\
-\boldsymbol{a}^{\mathrm{T}} & \mathbf{0}^{\mathrm{T}} & 0
\end{array}\right]-\sum_{j=1}^{\ell} w_{j} \hat{\Psi}_{j}-\sum_{i=1}^{3} s_{i} \hat{\Theta}_{i} \succeq O, \boldsymbol{w} \geq \mathbf{0}, \boldsymbol{e}_{1}^{\mathrm{T}} \boldsymbol{a}=1\right\}
$$

and $\varphi^{*}=\sqrt{-1 / a_{2}^{*}}$. Then $\varphi^{*} \leq t_{\min }$ holds for $t_{\min }$ defined in $(51)$, i.e. $\varphi^{*}$ corresponds to a lower bound for $\mu_{y} / \mu_{x}$. 


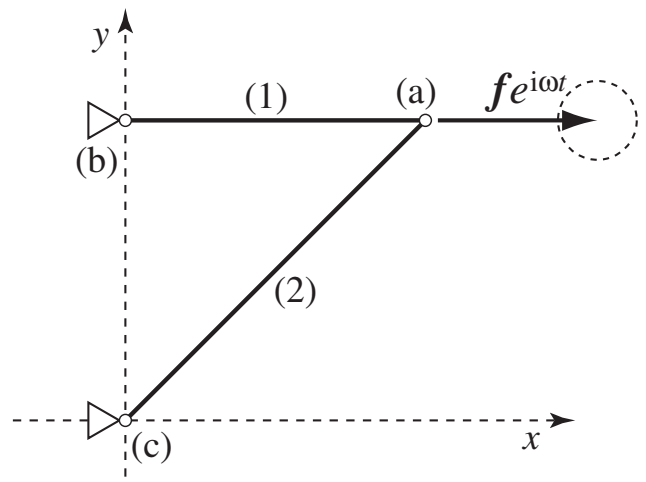

Figure 6: A 2-bar truss subjected to dynamic loading.

\section{$5 \quad$ Numerical experiments}

Conservative bounds for dynamic responses under the load uncertainties are found for various structures by solving SDP problems and SOCP problems. Computation has been carried out on Core 2 Duo (1.2 GHz with 2.0 GB memory) with MATLAB Ver. 7.5.0 [42]. We solve the SDP and SOCP problems by using SeDuMi Ver. 1.05 [38], which implements the primal-dual interior-point method for the linear programming problems over symmetric cones.

In the following examples, the elastic modulus is $200 \mathrm{GPa}$, and the mass density of members is $7.86 \times 10^{3} \mathrm{~kg} / \mathrm{m}^{3}$. In (8), we assume the complex damping, or the linear hysteretic damping, i.e. the damping matrix $C$ is given as

$$
\omega C=2 \beta K,
$$

where we put $\beta=0.02$ in the following examples.

\section{$5.1 \quad 2$-bar truss}

Consider a two-bar truss illustrated in Figure 6. The nodes (b) and (c) are pin-supported at $(x, y)=(0,100)$ and $(0,0)$ in $\mathrm{cm}$, respectively, while the node $(\mathrm{a})$ is free, i.e. $d=2$. The initial lengths of members (1) and (2) are $100 \mathrm{~cm}$ and $100 \sqrt{2} \mathrm{~cm}$, respectively.

As the nominal load, $\tilde{\boldsymbol{f}}$, the external force $10 \mathrm{kN}$ is applied in the positive direction of the $x$-axis at the node (a). In accordance with (5), the uncertainty model of the load is defined as

$$
\boldsymbol{f}=\tilde{\boldsymbol{f}}+\left[\begin{array}{l}
\zeta_{1} \\
\zeta_{2}
\end{array}\right], \quad \alpha \geq\|\boldsymbol{\zeta}\|, \quad j=1,2,
$$

where we put $F^{0}=1.0(\mathrm{kN}) \times I_{2}, \ell=1$, and $T_{1}=I_{2}$. Consequently, the uncertain load $\boldsymbol{f}$ is running through the circle depicted with the dashed lines in Figure 6. 


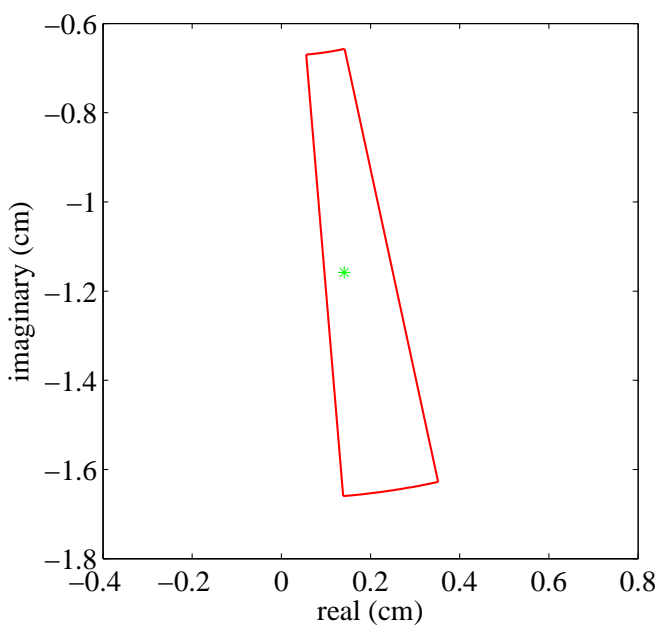

(i) $x$-direction

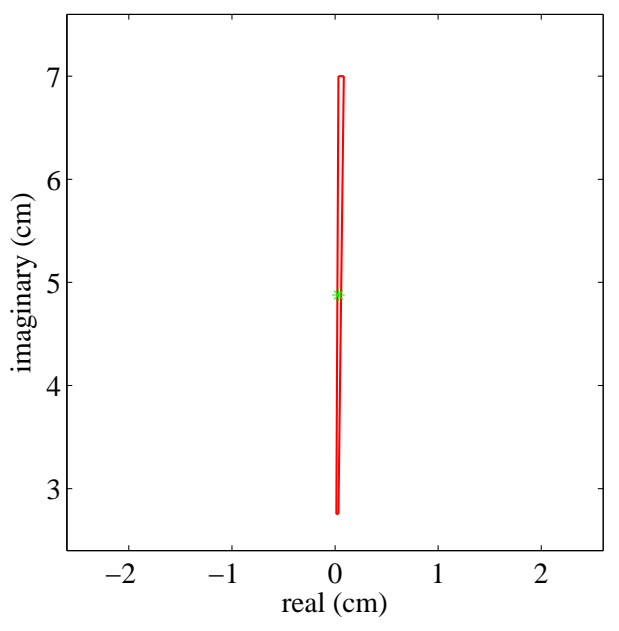

(ii) $y$-direction

Figure 7: Bounds for the complex amplitudes of the displacements of the 2-bar truss $\left(\omega=\omega_{1}^{0}\right) . *$ : nominal values; (i) displacement in the $x$-direction; (ii) displacement in the $y$-direction.
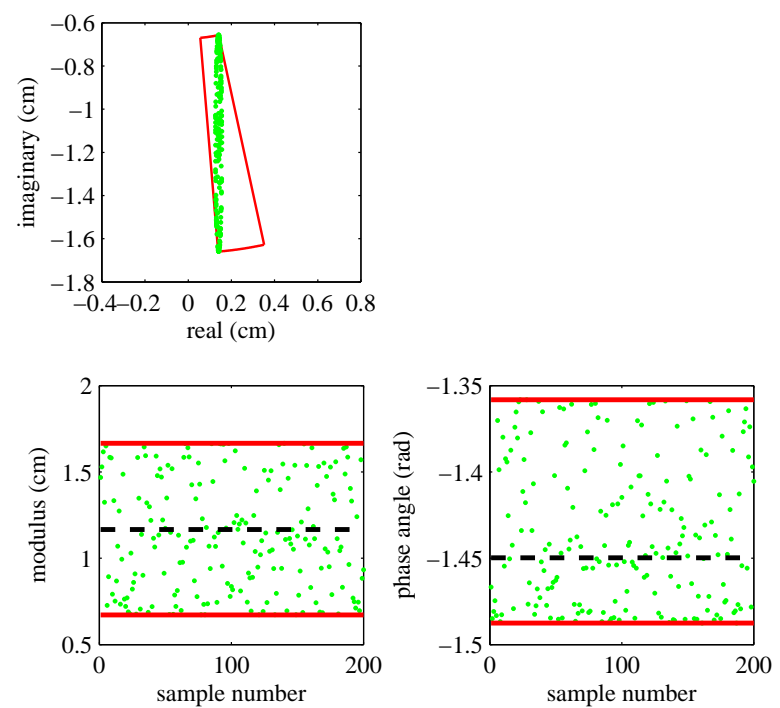

(i) $x$-direction
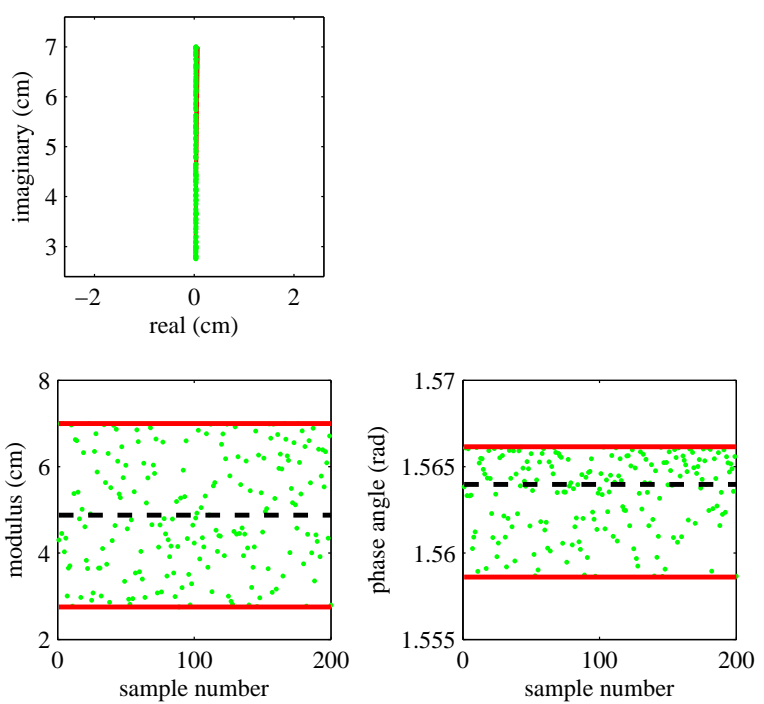

(ii) $y$-direction

Figure 8: Bounds and randomly generated samples for the member stresses of the 2-bar truss $\left(\omega=\omega_{1}^{0}\right)$. '-': bounds found in Figure 7; ' $--_{-}$': nominal values.

\subsubsection{Bounds for complex amplitude}

The bounds obtained are illustrated in Figure 7. The complex amplitude corresponding to the nominal load, $\tilde{\boldsymbol{f}}$, is

$$
\tilde{\boldsymbol{u}}=\left[\begin{array}{l}
u_{x} \\
u_{y}
\end{array}\right]=\left[\begin{array}{l}
0.141 \\
0.033
\end{array}\right]+\mathrm{i}\left[\begin{array}{c}
-1.158 \\
4.877
\end{array}\right],
$$

which is depicted in Figure 7 with $*$. 


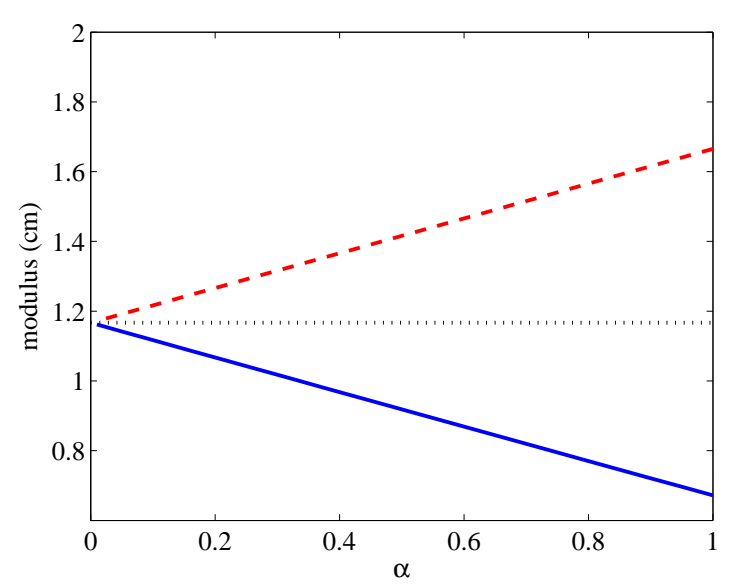

(i) modulus

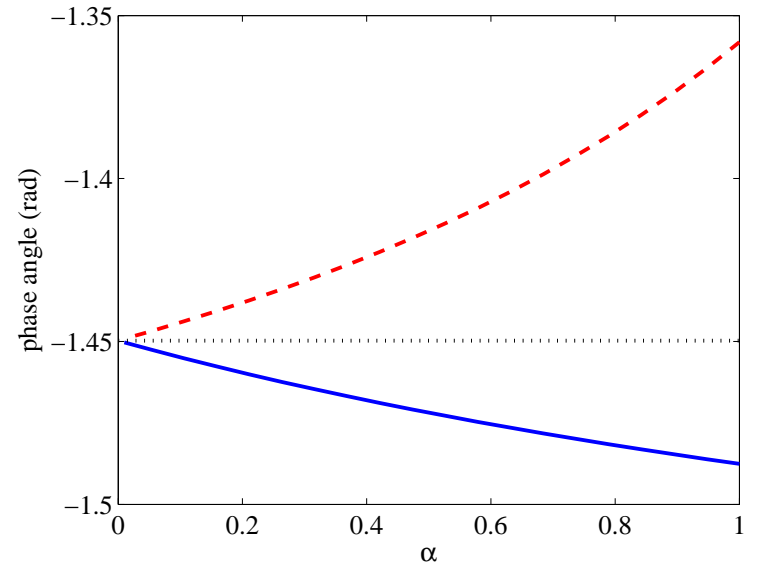

(ii) argument

Figure 9: Variations of bounds for the complex amplitudes of the displacements in the $x$-direction of the 2-bar truss with respect to the magnitude of uncertainty $\alpha$. (i) modulus of the amplitude; (ii) phase angle. '...'? nominal value; '-'? lower bounds; '- -': upper bounds.

We first choose $\omega=\omega_{1}^{0}$, where $\omega_{1}^{0}$ is the undamped fundamental natural circular frequency, and put $\alpha=1$. For the displacement in the $x$-direction illustrated in Figure 7 (i), the upper bound of the modulus of the complex amplitude is $1.665 \mathrm{~cm}$, which is obtained by solving the SDP problem (19); the minimum modulus is $0.672 \mathrm{~cm}$, which is obtained by solving the SOCP problem (21); the upper and lower bounds for the argument are $-1.358 \mathrm{rad}$ and $-1.488 \mathrm{rad}$, respectively, which are obtained by solving the SDP problems (33) and (35). Note that we solve (33) and (35) because $\tilde{u}_{x}$ exists in the forth quadrant. For the displacement in the $y$-direction (Figure 7 (ii)), upper and lower bounds for the argument are $1.566 \mathrm{rad}$ and $1.559 \mathrm{rad}$, respectively, which are obtained by solving the SDP problems (29) and (32). In summary, we obtain the bounds for $\boldsymbol{u}=\left(u_{x}, u_{y}\right)^{\mathrm{T}}$ as

$$
\begin{aligned}
& 0.672 \mathrm{~cm} \leq\left|u_{x}\right| \leq 1.665 \mathrm{~cm}, \quad-1.488 \mathrm{rad} \leq \operatorname{Arg} u_{x} \leq-1.358 \mathrm{rad}, \\
& 2.754 \mathrm{~cm} \leq\left|u_{y}\right| \leq 7.000 \mathrm{~cm}, \quad 1.559 \mathrm{rad} \leq \operatorname{Arg} u_{y} \leq 1.566 \mathrm{rad} .
\end{aligned}
$$

The system is almost in the quadrature with the driving load, but $u_{x}$ and $u_{y}$ are in anti-phase with each other, because $\operatorname{Arg} u_{x} \simeq-\pi / 2$ and $\operatorname{Arg} u_{y} \simeq \pi / 2$.

In order to verify these results, we randomly generate a number of loads, $\boldsymbol{f}$, satisfying (54), and compute the corresponding amplitudes. The obtained displacements are shown by a lot of points in Figure 8 with the bounds obtained. It is observed in Figure 8 that all generated amplitudes are included in the bounds, which confirms that the obtained bounds correspond to confidential, or outer, approximations of the sets of complex amplitudes. In addition, we can also see that all bounds are sufficiently tight.

We next investigate the dependence of the distribution of $\boldsymbol{u}$ on the system parameters. Figure 9 depicts the variation of $\left|u_{x}\right|$ and $\operatorname{Arg} u_{x}$ with respect to the magnitude of uncertainty, $\alpha$. In Figure 10, we illustrate the bounds of $u_{x}$ and $u_{y}$ for various $\omega$, as well as the samples generated randomly. From Figure 10 we can observe the resonant behavior at $\omega=\omega_{2}^{0}$, where $u_{x}$ and $u_{y}$ are in the same phase. In Figure 10 (iii) and Figure 10 (iv) we see that the system is in phase with the driving load, i.e. $\operatorname{Arg} u_{x} \simeq 0$ and $\operatorname{Arg} u_{y} \simeq 0$. 


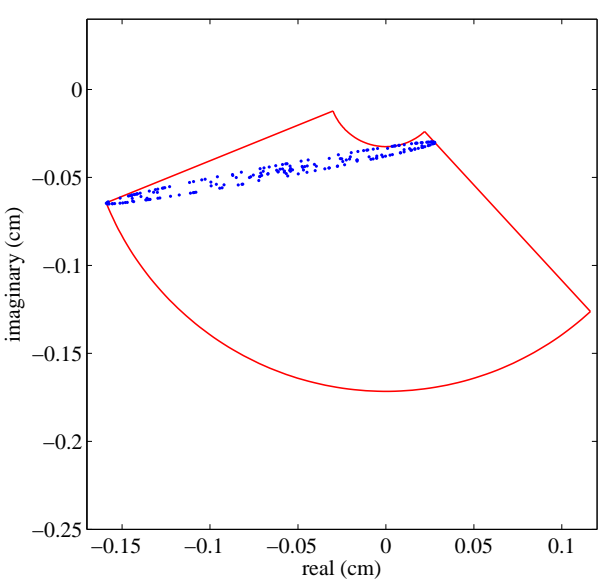

(i) $x$-direction $\left(\omega=1.1 \omega_{1}^{0}\right)$

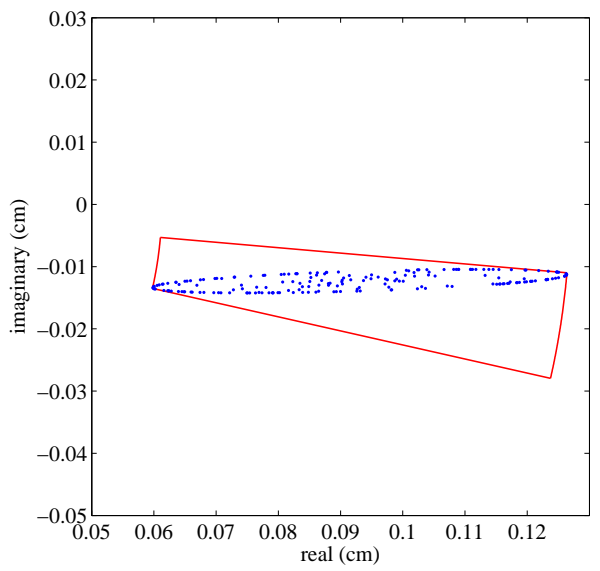

(iii) $x$-direction $\left(\omega=1.3 \omega_{1}^{0}\right)$

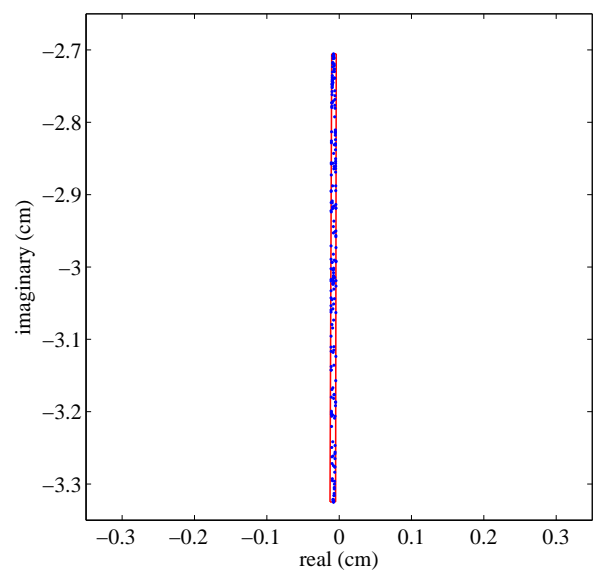

(v) $x$-direction $\left(\omega=\omega_{2}^{0}\right)$

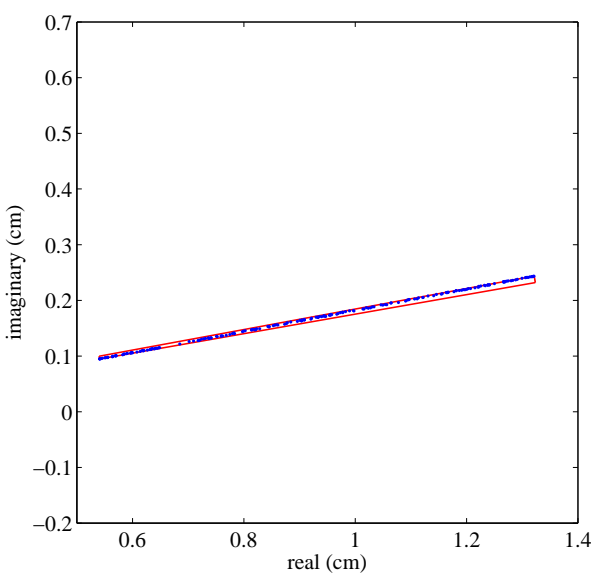

(ii) $y$-direction $\left(\omega=1.1 \omega_{1}^{0}\right)$

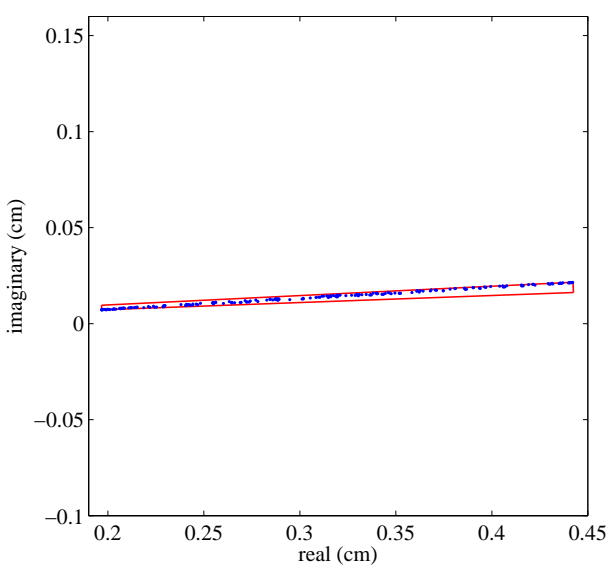

(iv) $y$-direction $\left(\omega=1.3 \omega_{1}^{0}\right)$

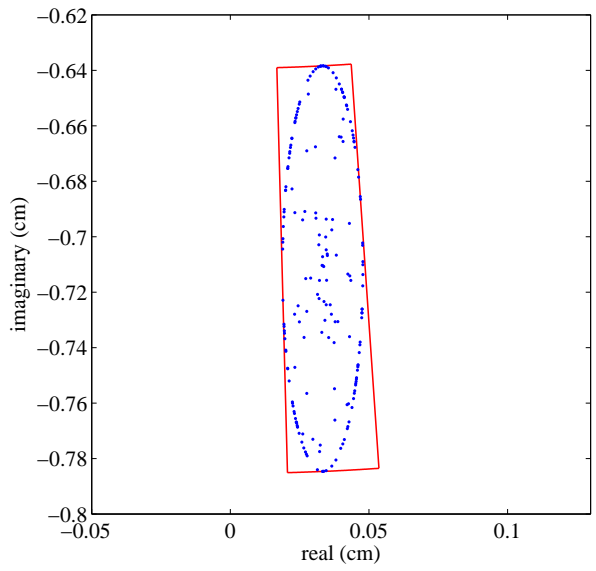

(vi) $y$-direction $\left(\omega=\omega_{2}^{0}\right)$

Figure 10: Bounds and randomly generated samples for the complex amplitudes of the displacements of the 2-bar truss. 


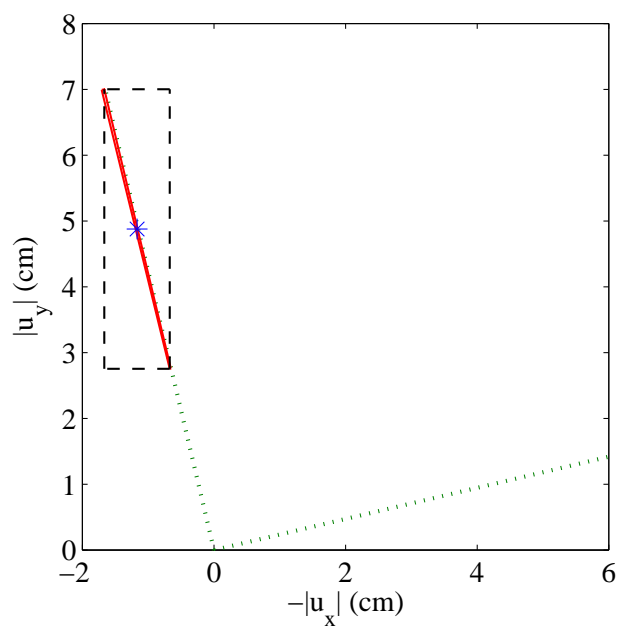

(i) $\omega=\omega_{1}^{0}$

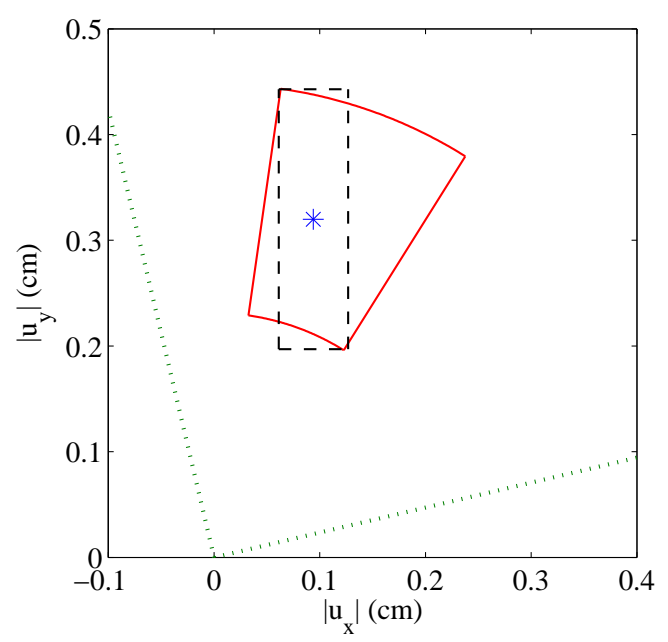

(iii) $\omega=1.3 \omega_{1}^{0}$

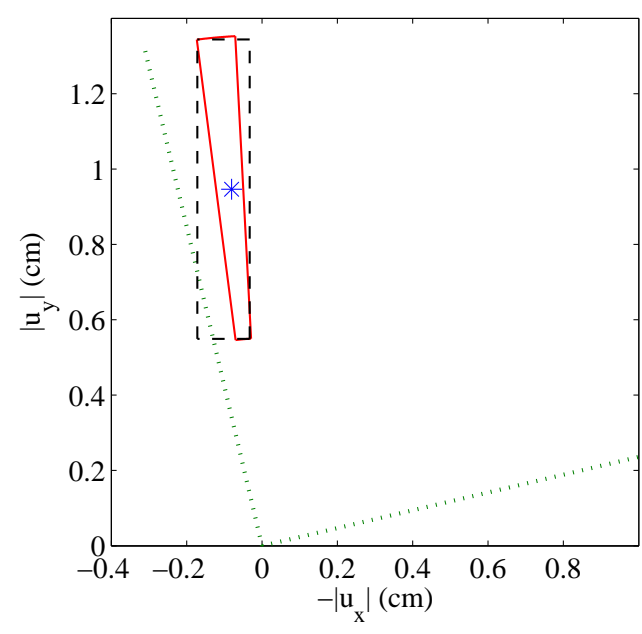

(ii) $\omega=1.1 \omega_{1}^{0}$

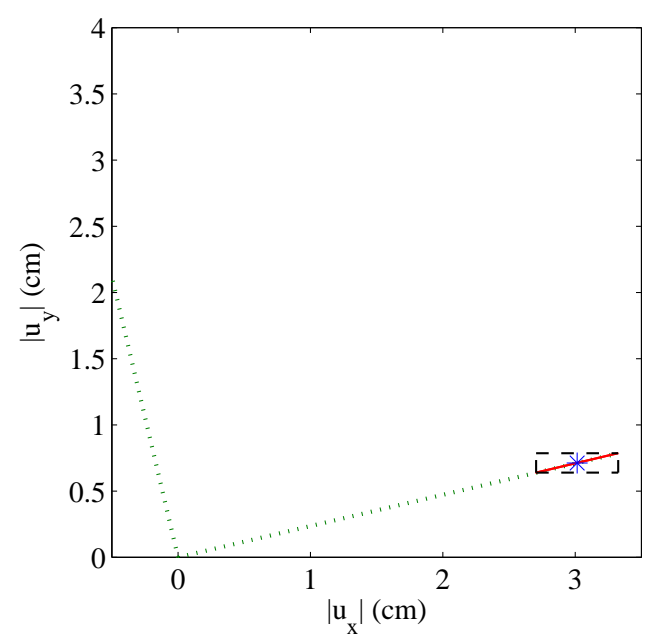

(iv) $\omega=\omega_{2}^{0}$

Figure 11: Bounds for the nodal oscillation of the 2-bar truss. $*$ : nominal values; '-': bounds obtained by solving (44), (45), (50), and (53); '- -': bounds obtained by solving (19) and (21); '...': directions of eigenmodes without damping.

\subsubsection{Bounds for nodal oscillation}

We next consider the distribution of the vector $\boldsymbol{\nu}=\left(\left|u_{x}\right|,\left|u_{y}\right|\right)^{\mathrm{T}}$, as is investigated in section 4 . Upper and lower bounds for $\sqrt{\left|u_{x}\right|^{2}+\left|u_{y}\right|^{2}}$ are obtained by solving (44) and (45), respectively; upper and lower bounds for $\left|u_{y}\right| /\left|u_{x}\right|$ are obtained by solving (50) and (53), respectively. The obtained bounds are shown in Figure 11 for various $\omega$. Here, the vector $\left(\left|u_{x}\right|,\left|u_{y}\right|\right)$ exists in the intersection of the two sets shown with the solid and dashed lines, as discussed in Remark 4.1. Note that we plot $-\left|u_{x}\right|$ and $\left|u_{y}\right|$ in Figures 11 (i) and (ii), because we can see from Figure 7 and Figure 10 that $u_{x}$ is in anti-phase with $u_{y}$. In contrast, we plot $\left|u_{x}\right|$ and $\left|u_{y}\right|$ in Figures 11 (iii) and (iv).

The directions of two eigenmodes are also depicted in Figure 11. We can see the resonances in Figure 11 (i) and (iv). From Figure 12 we can confirm that the bounds shown in Figure 11 are confidential and sufficiently tight. 


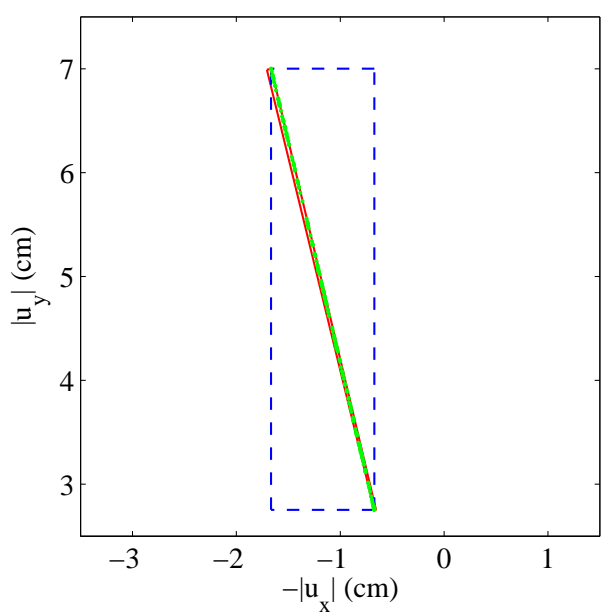

(i) $\omega=\omega_{1}^{0}$

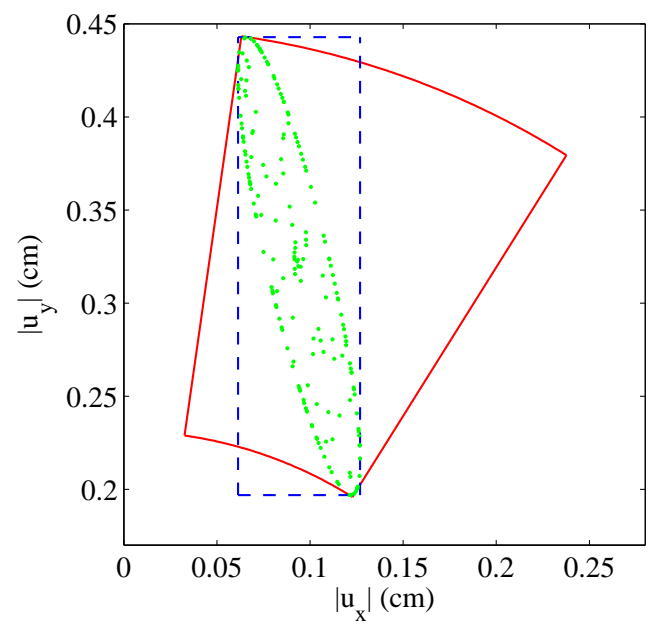

(iii) $\omega=1.3 \omega_{1}^{0}$

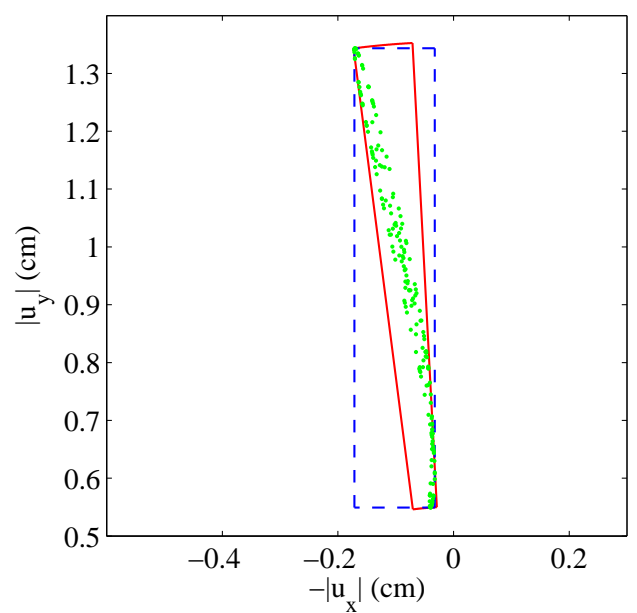

(ii) $\omega=1.1 \omega_{1}^{0}$

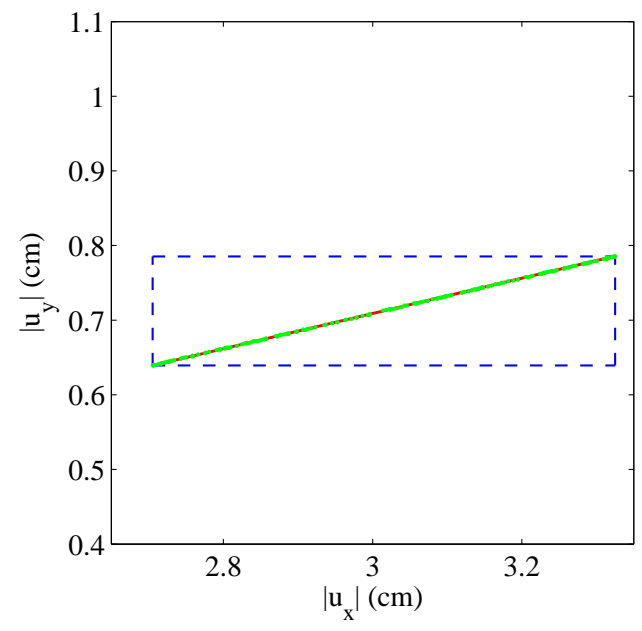

(iv) $\omega=\omega_{2}^{0}$

Figure 12: Bounds and randomly generated samples for the nodal oscillation of the 2-bar truss.

\subsubsection{Bounds for member stress}

As discussed in Remark 3.17, the distribution of (the complex amplitudes of) the member stresses is investigated in Figure 13 and Figure 14 by using the SDP and SOCP formulations presented in section 3. It is observed in Figure 13 that the stresses of both members are in anti-phase with the driving load of $\omega=\omega_{1}^{0}$. From Figure 14 we see that the stress of the member (2) is in phase with the driving load of $\omega=1.1 \omega_{1}^{0}$, while there exists a case in which the stress of the member (1) is in anti-phase with the driving load, i.e. the phase angle of the member (1) has very large uncertainty.

\section{$5.2 \quad 29$-bar truss}

Next we consider a 29-bar truss shown in Figure 1. The lengths of members in the directions of the $x$ - and $y$-axes are $50 \mathrm{~cm}$ and $100 \mathrm{~cm}$, respectively.

As the nominal load, $\tilde{\boldsymbol{f}}$, the external forces $12 \mathrm{kN}$ and $8 \mathrm{kN}$ are applied at the nodes (a) and (b), respectively, in the negative direction of $y$-axis. The driving frequency is $\omega=\omega_{1}^{0}$. The uncertainty 

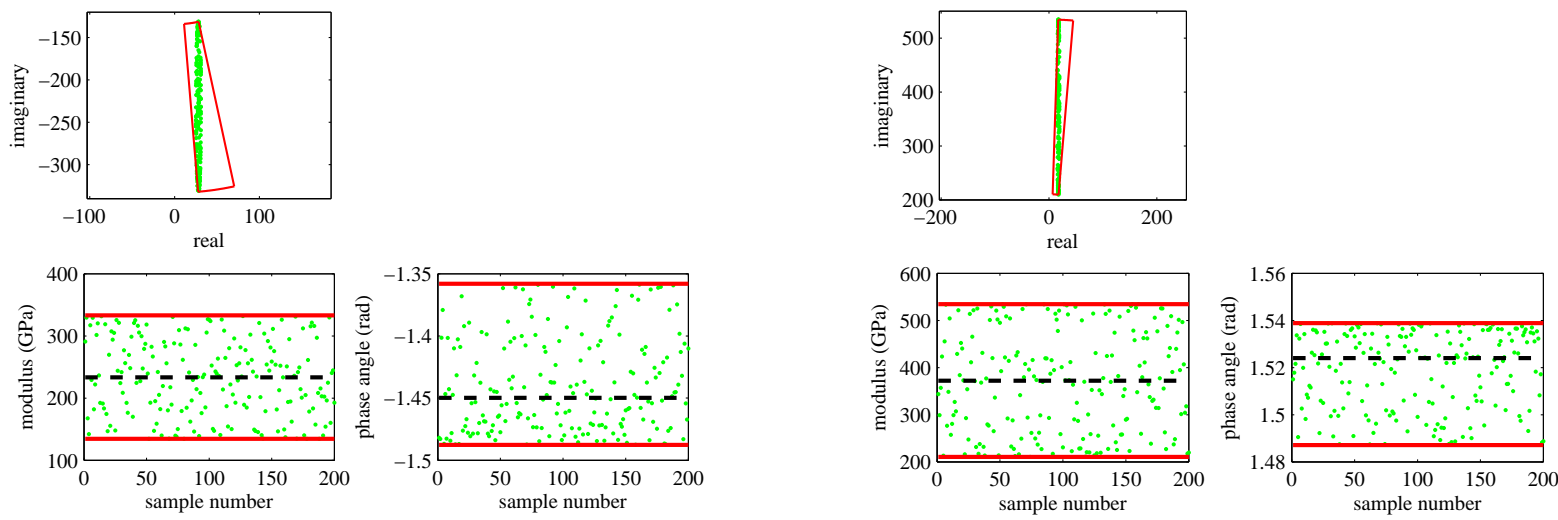

(i) member (1)

(ii) member (2)

Figure 13: Bounds and randomly generated samples for the member stresses of the 2-bar truss $\left(\omega=\omega_{1}^{0}\right)$. '-': bounds; '--': nominal values.
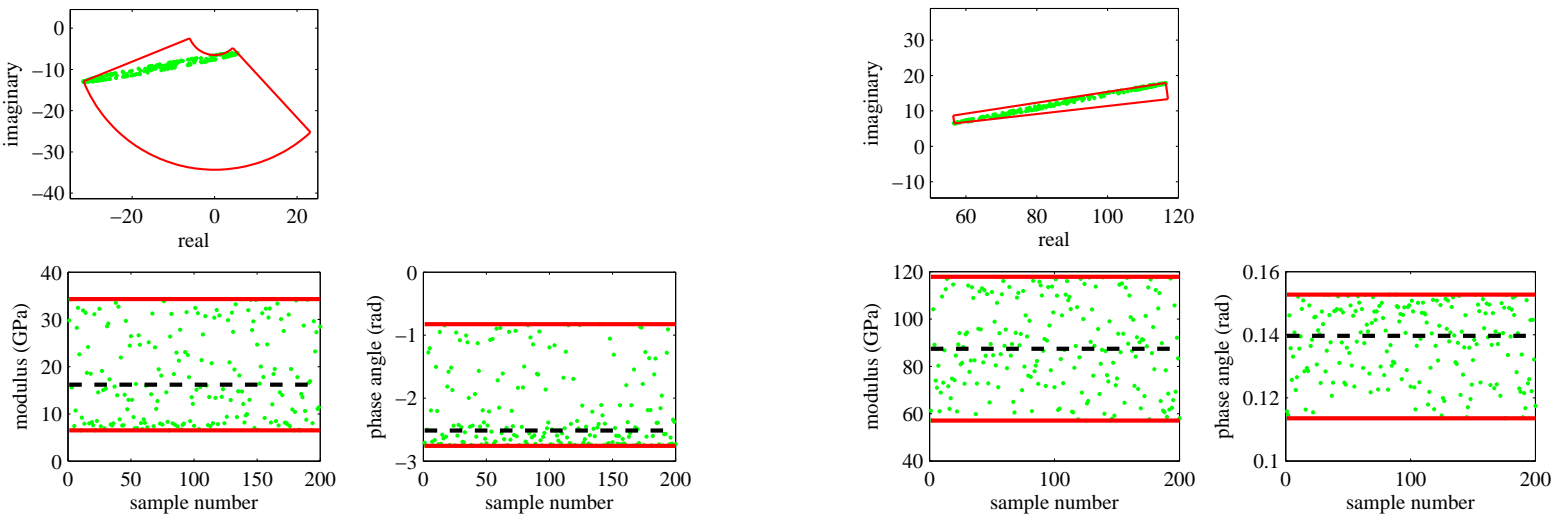

(i) member (1)

(ii) member (2)

Figure 14: Bounds and randomly generated samples for the member stresses of the 2-bar truss $\left(\omega=1.1 \omega_{1}^{0}\right)$. '-': bounds; '--': nominal values.

model of $\boldsymbol{f},(5)$, is defined as discussed in Example 2.1 with $\bar{f}_{0}=1 \mathrm{kN}$. Consequently, the external forces are running through the circles depicted with the dotted lines in Figure 1. Note that uncertain forces may possibly exist at all free nodes, and any two sets of the uncertain loads have no relation.

We investigate the displacement of the node (a), which is denoted by $\left(u_{x}, u_{y}\right)$. We put $\alpha=1.0$ in (5). For the amplitudes in the $x$ - and $y$-th directions, we obtain the bounds of the moduli and phase angles, which are shown in Figure 15. The obtained bounds are confirmed in Figure 16 to be conservative and sufficiently tight. Variations of $\left|u_{x}\right|,\left|u_{y}\right|, \operatorname{Arg} u_{x}$, and $\operatorname{Arg} u_{y}$ with respect to the magnitude of uncertainty, $\alpha$, are depicted in Figure 17. Figure 18 illustrates the variations of $\left|u_{x}\right|$ and $\left|u_{y}\right|$ with respect to the frequency of the driving load, $\omega$, in the range of $\omega \in\left[\omega_{1}^{0}, \omega_{2}^{0}\right]$, where $\omega_{1}^{0}=1.248 \mathrm{rad} / \mathrm{s}$ and $\omega_{2}^{0}=1.872 \mathrm{rad} / \mathrm{s}$. It is observed from Figure 18 (i) that the minimum value, $r_{\min }$, of $\left|u_{x}\right|$ is $r_{\min }=0$ for $\omega \in\left[1.76, \omega_{2}^{0}\right]$ as discussed in Remark 3.9.

For the node (a), Figure 19 depicts the bounds for the distribution of the vector $\left(\left|u_{x}\right|,\left|u_{y}\right|\right)$, which are confirmed in Figure 20. It is observed from Figure 19 (i) that the driving force with $\omega=\omega_{1}^{0}$ yields the resonance, where the oscillation is in the direction of the first eigenmode. In contrast, 


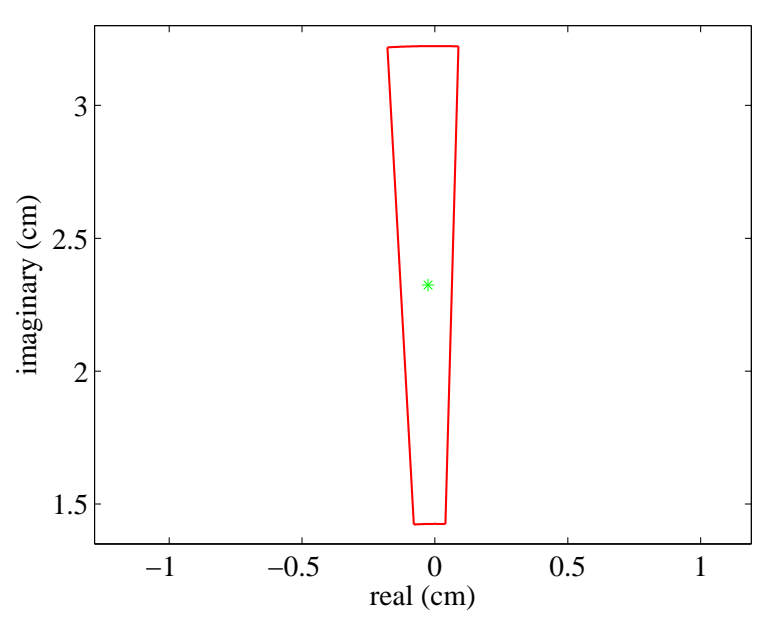

(i) $x$-direction

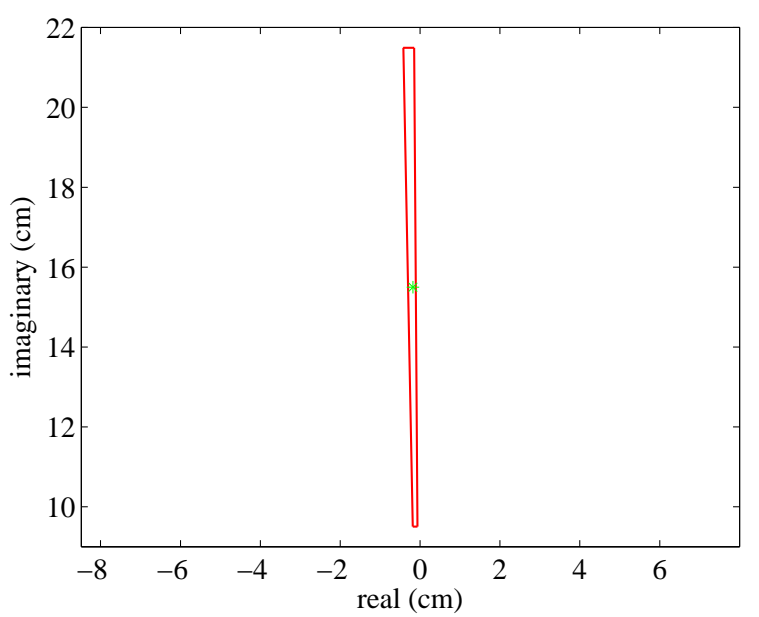

(ii) $y$-direction

Figure 15: Bounds for the complex amplitudes of the displacements of the 29-bar truss $\left(\omega=\omega_{1}^{0}\right)$. *: nominal values; (i) displacement in the $x$-direction; (ii) displacement in the $y$-direction.

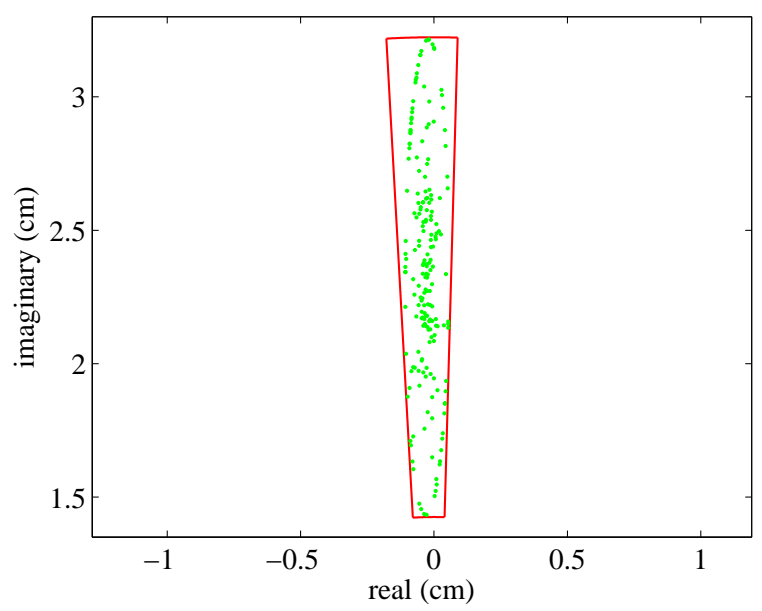

(i) $x$-direction

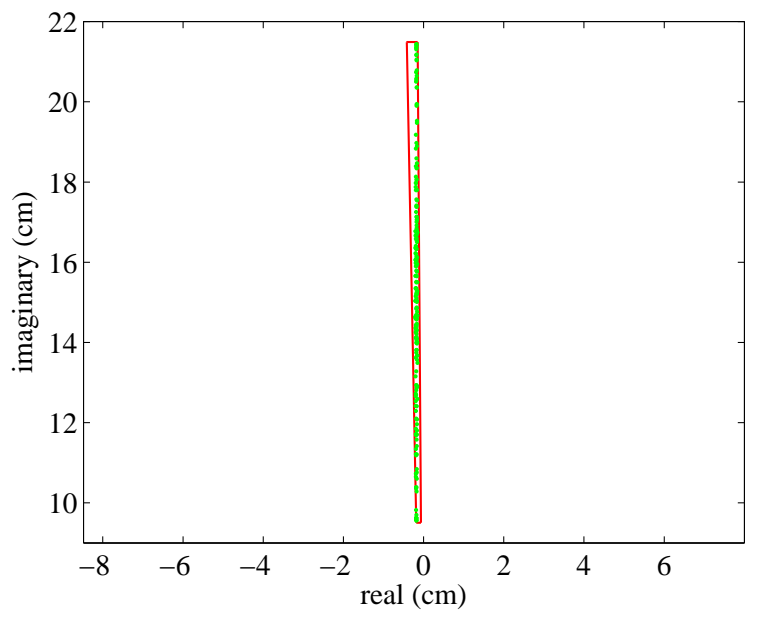

(ii) $y$-direction

Figure 16: Bounds and randomly generated samples for the complex amplitudes of the displacements of the 29-bar truss $\left(\omega=\omega_{1}^{0}\right)$.

in Figure 19 (iv) with $\omega=\omega_{2}^{0}$, the uncertainty of the oscillation direction, $\left|u_{y}\right| /\left|u_{x}\right|$, is very large, although there exists a case in which the oscillation is approximately in the direction of the second eigenmode. It should be emphasized that in such a case the obtained bounds are very tight, which can be seen in Figure 20 (iv). Figure 21 illustrates the variations of $\sqrt{\left|u_{x}\right|^{2}+\left|u_{y}\right|^{2}}$ with respect to $\alpha$ and $\omega$. 


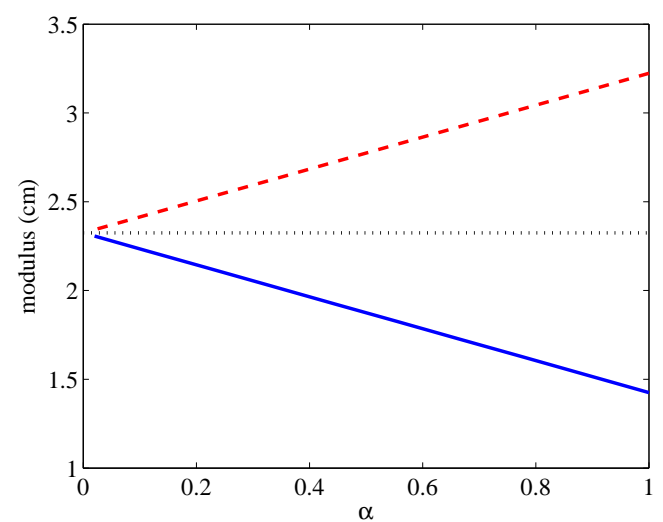

(i) modulus of the $x$-direction

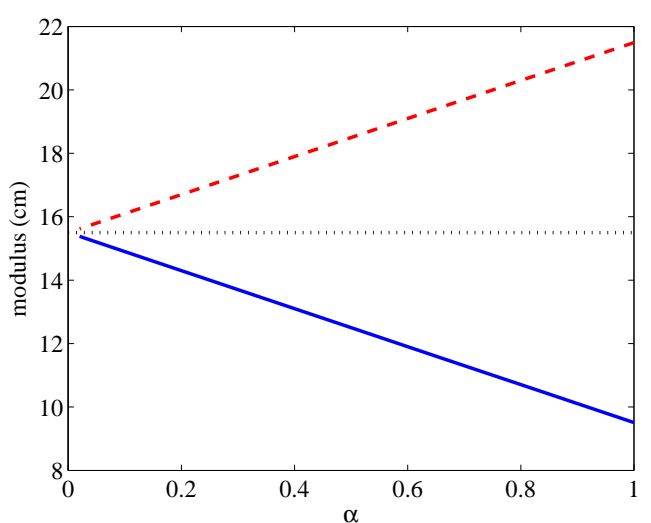

(iii) modulus of the $y$-direction

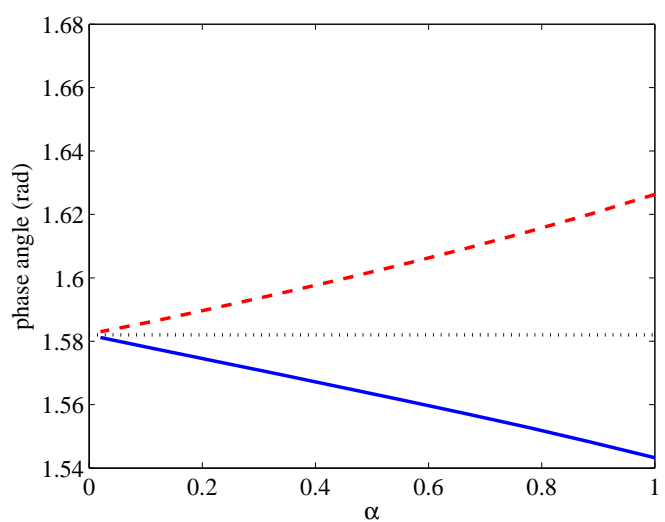

(ii) argument of the $x$-direction

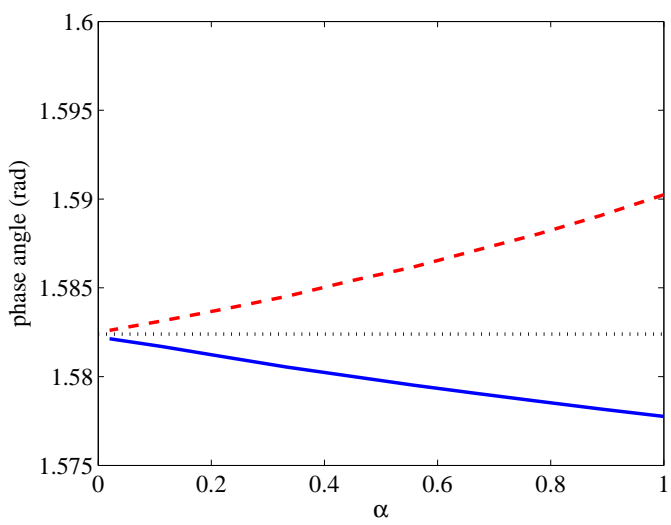

(iv) argument of the $y$-direction

Figure 17: Variations of bounds for the complex amplitudes of the displacements in the $x$-direction of the 29-bar truss with respect to the magnitude of uncertainty $\alpha$. '...'? nominal value; '-': lower bounds; '--': upper bounds.

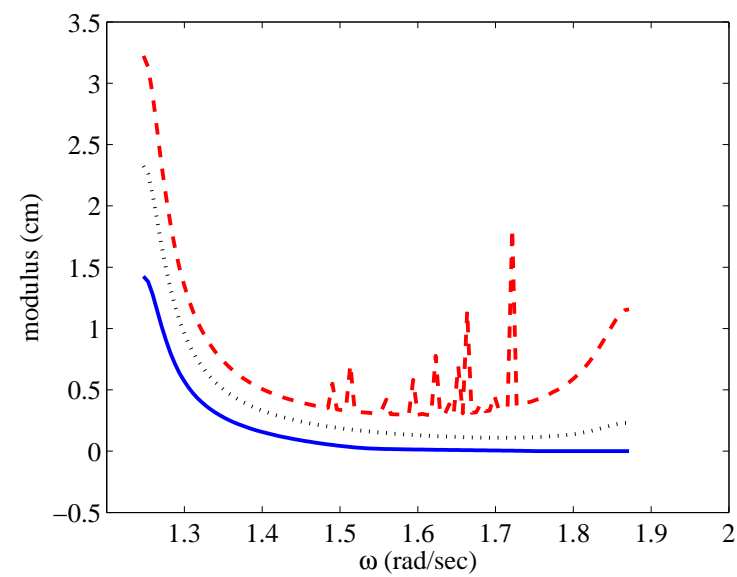

(i) $x$-direction

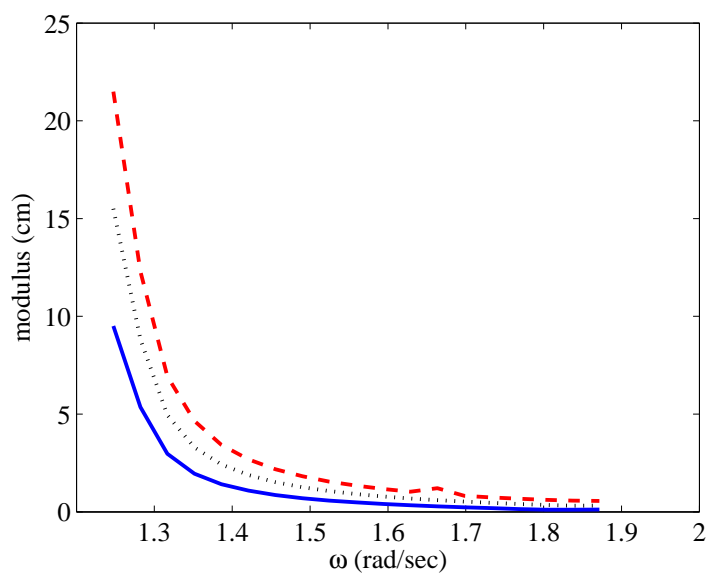

(ii) $y$-direction

Figure 18: Variations of bounds for the complex amplitudes of the displacements in the $x$-direction of the 29-bar truss with respect to the frequency $\omega$ of the driving force. '.. ': nominal value; '- ': lower bounds; '- -': upper bounds. 


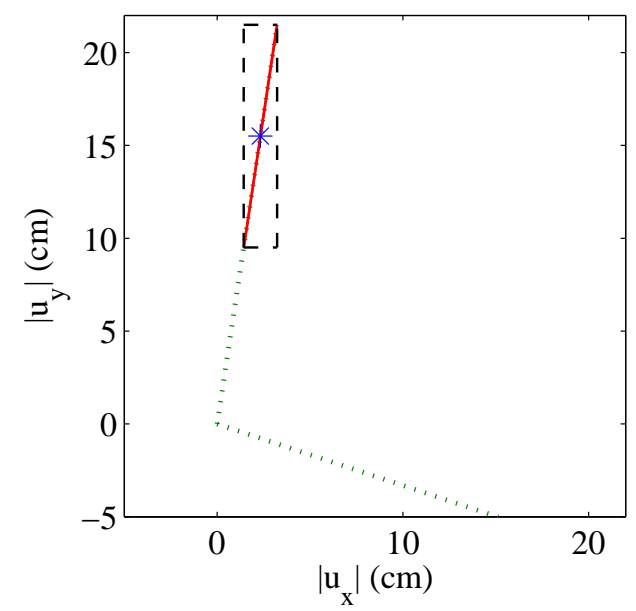

(i) $\omega=\omega_{1}^{0}$

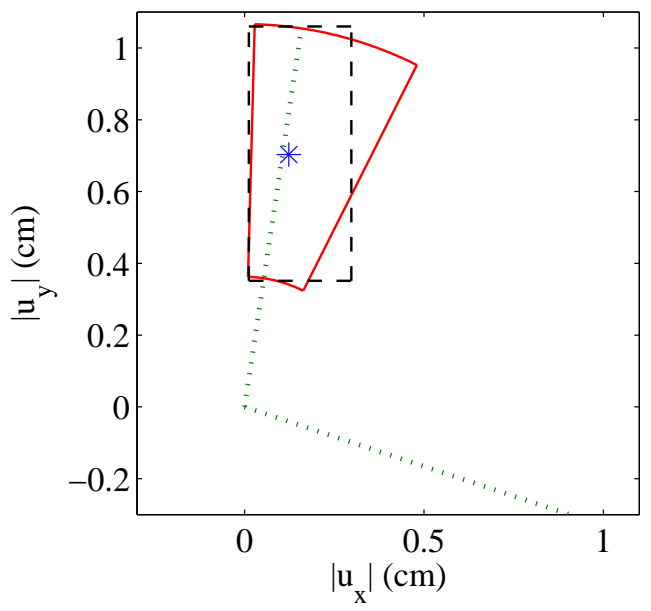

(iii) $\omega=1.3 \omega_{1}^{0}$

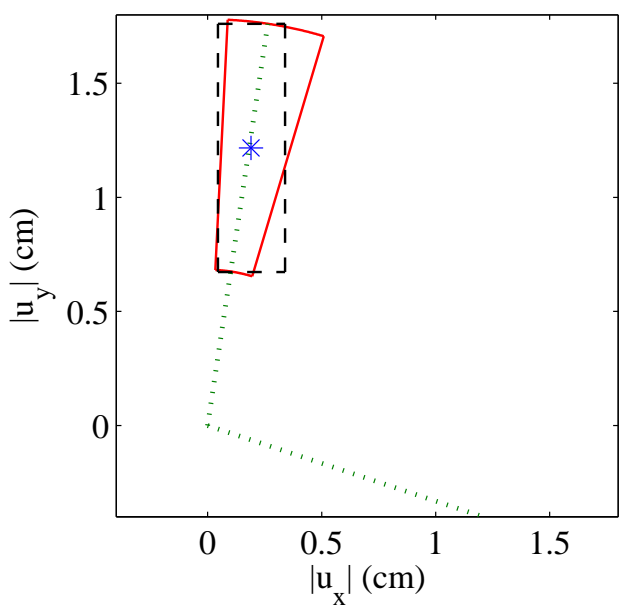

(ii) $\omega=1.2 \omega_{1}^{0}$

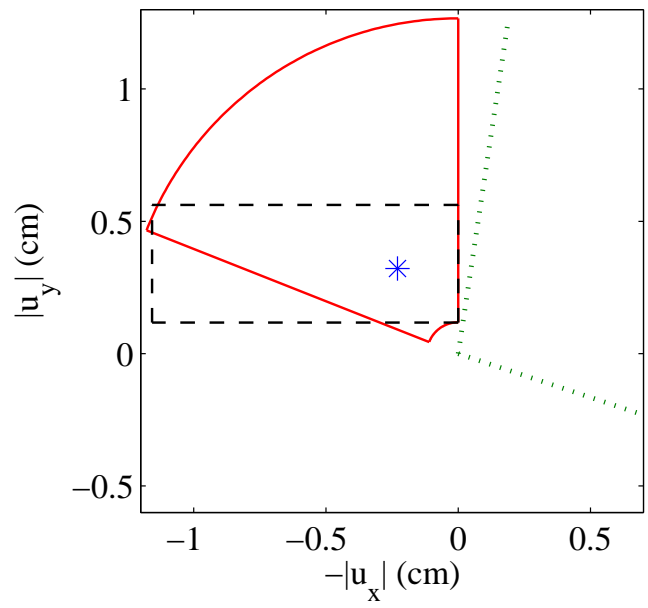

(iv) $\omega=\omega_{2}^{0}$

Figure 19: Bounds for the nodal oscillation of the 29-bar truss. $*$ : nominal values; '-': bounds obtained by solving (44), (45), (50), and (53); '- -': bounds obtained by solving (19) and (21); '...': directions of eigenmodes without damping.

\section{$6 \quad$ Further generalization of uncertainty model}

\subsection{Formulations for generalized uncertainty model}

Recall that we have assumed in sections 3 and 4 that the matrix $F_{0} \in \mathbb{R}^{d \times k}$ defining the uncertainty set, (5), is nonsingular. In this section we consider formulations for confidence bounds without this assumption. Note that the matrices $\Psi_{1}, \ldots, \Psi_{\ell}$ defined in Proposition 3.4 includes $F_{0}^{-1}$, hence formulations presented in sections 3.1, 3.3, 4.1, and 4.2 are not valid for a singular $F_{0}$. In contrast, the SOCP formulation in section 3.2 can be applied to a singular $F_{0}$ in order to find a lower bound for the modulus of the displacement amplitude.

As investigated in Example 2.1 and Example 2.2, if $F_{0}$ is nonsingular, then uncertain forces can exist at all free nodes. By using a singular $F_{0}$, it is possible to consider the uncertainty model where uncertain forces can exist only at specified nodes. 


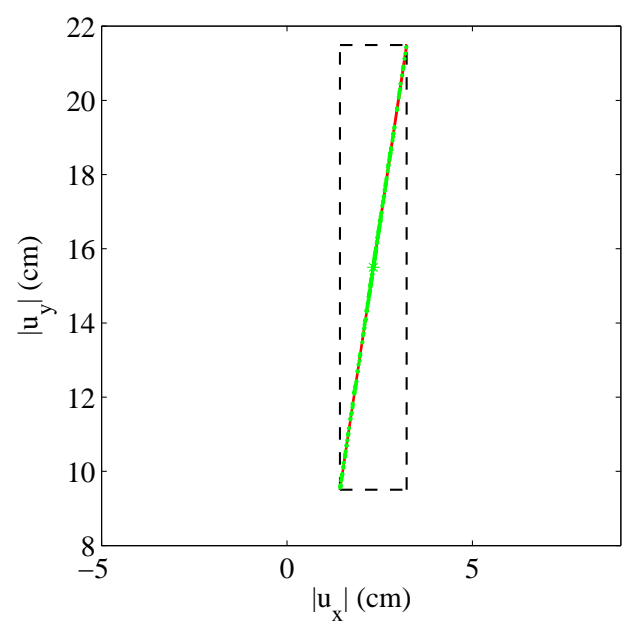

(i) $\omega=\omega_{1}^{0}$

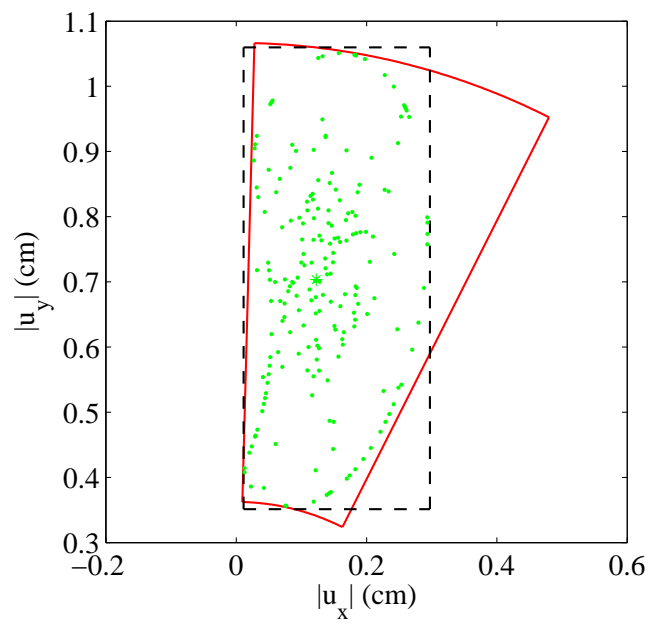

(iii) $\omega=1.3 \omega_{1}^{0}$

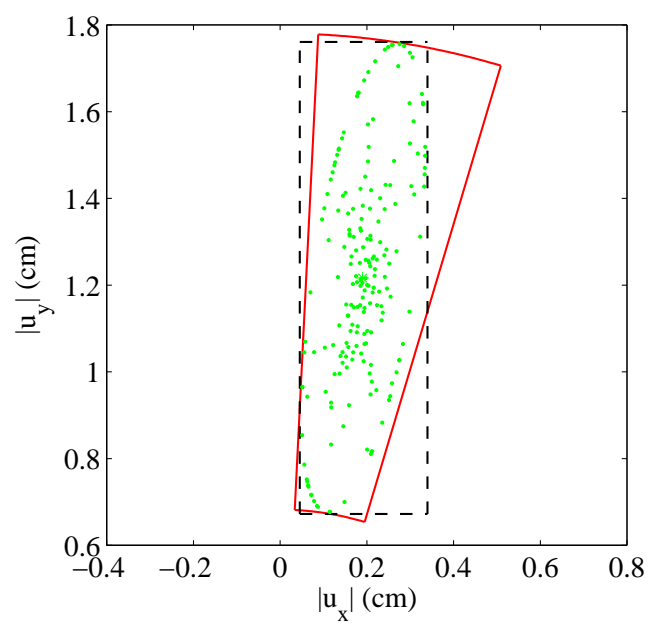

(ii) $\omega=1.2 \omega_{1}^{0}$

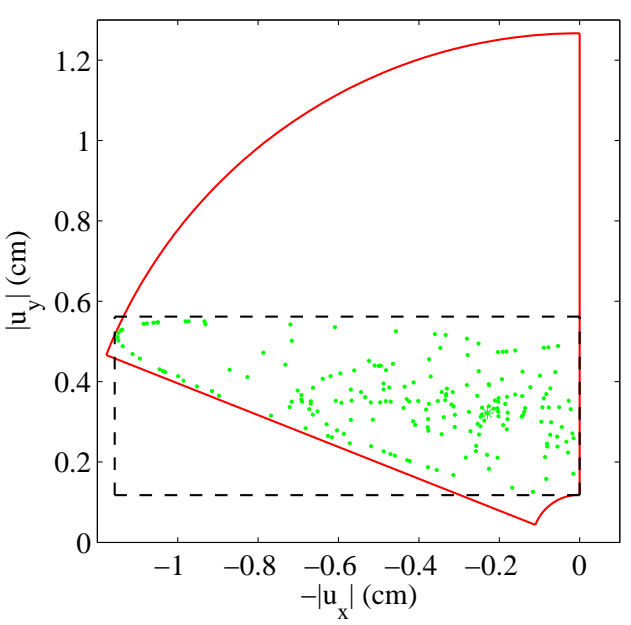

(iv) $\omega=\omega_{2}^{0}$

Figure 20: Bounds and randomly generated samples for the nodal oscillation of the 29-bar truss.

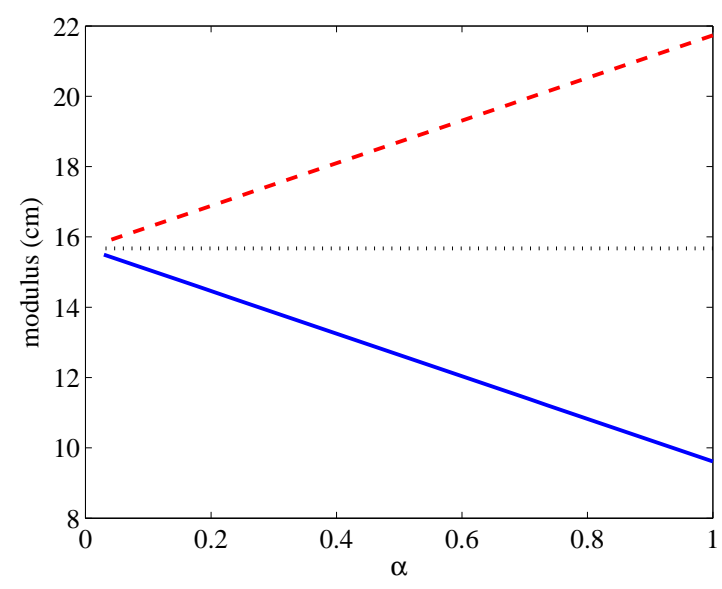

(i) $\alpha$

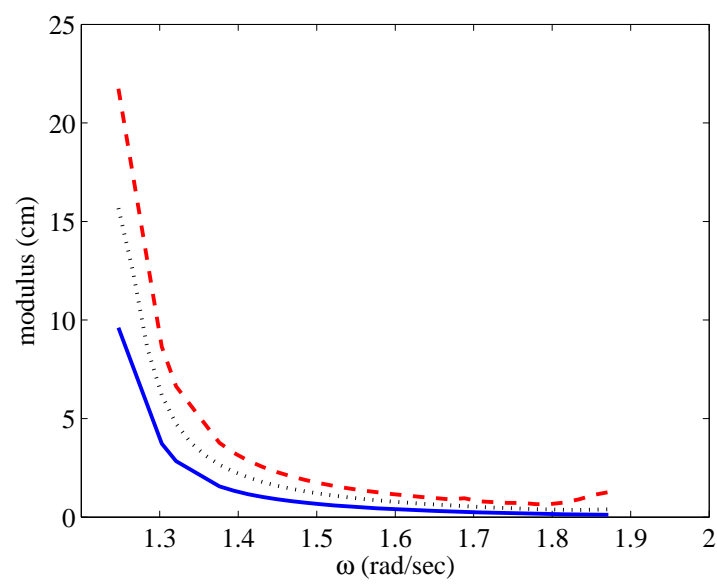

(ii) $\omega$

Figure 21: Variations of bounds for the nodal oscillation of the 29-bar truss with respect to $\alpha$ and $\omega$. ' '..': nominal value; '-': lower bounds; '- -': upper bounds. 


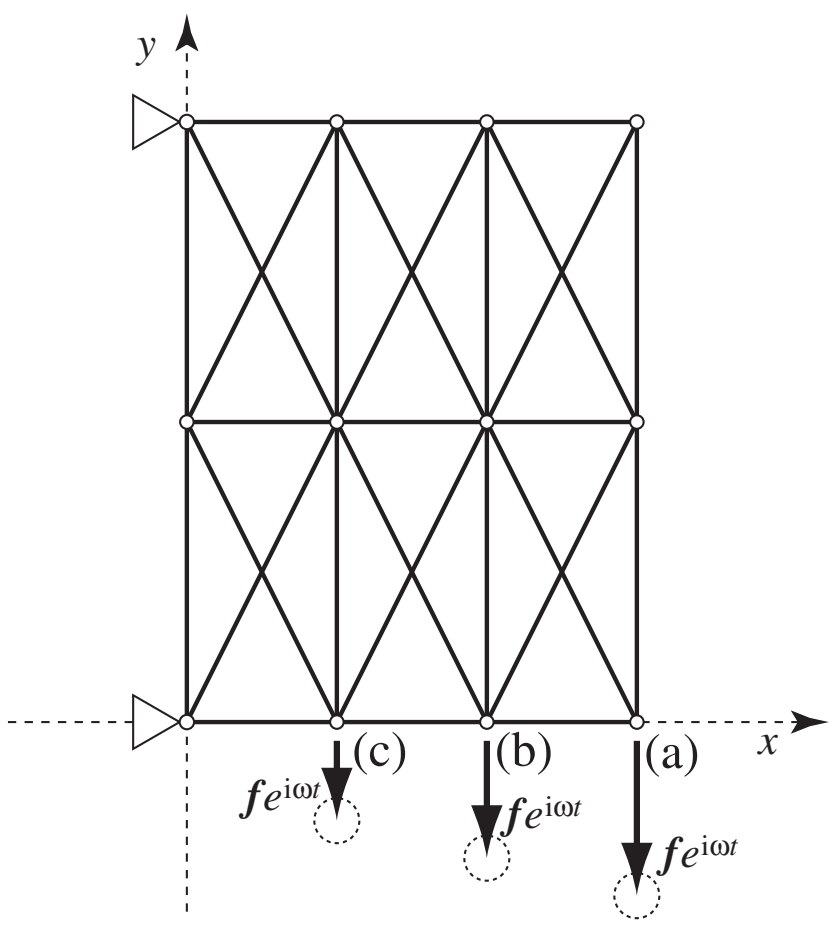

Figure 22: 29-bar truss under the uncertain dynamic load defined with a singular $F_{0}$ in (5).

Example 6.1. Consider a plane truss illustrated in Figure 22. The nominal harmonic forces are applied at the nodes (a)-(c). In contrast to Figure 1, suppose that uncertain forces can exist only at the nodes (a)-(c). Such an uncertainty model can be represented as (5) by putting

$$
\begin{aligned}
& F_{0}=\bar{f}_{0}\left[\begin{array}{l}
I_{6} \\
O
\end{array}\right] \in \mathbb{R}^{20 \times 6}, \\
& T_{1}=\left[\begin{array}{lll}
I_{2} & O & O
\end{array}\right], \quad T_{2}=\left[\begin{array}{lll}
O & I_{2} & O
\end{array}\right], \quad T_{3}=\left[\begin{array}{lll}
O & O & I_{2}
\end{array}\right] \in \mathbb{R}^{2 \times 6},
\end{aligned}
$$

where $k=6$ and $\ell=3$. Thus we see that $F_{0}$ is singular.

Proposition 6.2. Suppose that $F_{0}$ in (5) is singular. For $\mathcal{V}$ defined in (12), $\boldsymbol{v} \in \mathcal{V}$ if and only if $\boldsymbol{v}$ satisfies

$$
\begin{aligned}
& {\left[\begin{array}{l}
\boldsymbol{v} \\
1
\end{array}\right]^{\mathrm{T}} \check{\Psi}_{j}\left[\begin{array}{l}
\boldsymbol{v} \\
1
\end{array}\right] \geq 0, \quad j=1, \ldots, \ell,} \\
& {\left[\begin{array}{l}
\boldsymbol{v} \\
1
\end{array}\right]^{\mathrm{T}} \check{\Theta}\left[\begin{array}{l}
\boldsymbol{v} \\
1
\end{array}\right]=0,}
\end{aligned}
$$

where $\check{\Psi}_{j}(j=1, \ldots, \ell)$ and $\check{\Theta}$ are constant matrices defined by

$$
\begin{aligned}
& \check{\Psi}_{j}=\operatorname{diag}\left(\mathbf{0}, \alpha^{2}\right)-\left[\begin{array}{c}
S_{1}^{\mathrm{T}} \\
-\tilde{\boldsymbol{f}}^{\mathrm{T}}
\end{array}\right]\left(T_{j} F_{0}^{\dagger}\right)^{\mathrm{T}} T_{j} F_{0}^{\dagger}\left[\begin{array}{ll}
S_{1} & -\tilde{\boldsymbol{f}}
\end{array}\right], \quad j=1, \ldots, \ell, \\
& \check{\Theta}=-\left[\begin{array}{cc}
\left(\left(F_{0}^{\perp}\right)^{\mathrm{T}} S_{1}\right)^{\mathrm{T}} & S_{2}^{\mathrm{T}} \\
-\left(\left(F_{0}^{\perp}\right)^{\mathrm{T}} \tilde{\boldsymbol{f}}\right)^{\mathrm{T}} & O
\end{array}\right]\left[\begin{array}{cc}
\left(F_{0}^{\perp}\right)^{\mathrm{T}} S_{1} & -\left(F_{0}^{\perp}\right)^{\mathrm{T}} \tilde{\boldsymbol{f}} \\
S_{2} & O
\end{array}\right],
\end{aligned}
$$


where $F_{0}^{\dagger} \in \mathbb{R}^{k \times d}$ is the Moore-Penrose pseudoinverse of $F_{0}, F_{0}^{\perp} \in \mathbb{R}^{d \times\left(d-\operatorname{rank} F_{0}\right)}$ the basis for the left null space of $F_{0}$, and rank $F_{0}$ the row rank of $F_{0}$.

The proof appears in appendix A.5.

Example 6.3. In the case of Example 6.1, the matrices in Proposition 6.2 are obtained as

$$
F_{0}^{\dagger}=\frac{1}{\bar{f}_{0}}\left[\begin{array}{ll}
I_{6} & O
\end{array}\right] \in \mathbb{R}^{6 \times 20}, \quad F_{0}^{\perp}=\left[\begin{array}{c}
O \\
I_{14}
\end{array}\right] \in \mathbb{R}^{20 \times 14} .
$$

where $\operatorname{rank} F_{0}=6$.

It follows from Proposition 6.2 that the SDP formulations presented in sections 3.1, 3.3, 4.1, and 4.2 are generalized for a singular $F_{0}$ by replacing $\Psi_{1}, \ldots, \Psi_{\ell}$ and $\Theta$ with $\check{\Psi}_{1}, \ldots, \check{\Psi}_{\ell}$ and $\check{\Theta}$, respectively.

\subsection{Numerical example for generalized uncertainty model}

Consider a 29-bar truss shown in Figure 22. As the nominal load, $\tilde{\boldsymbol{f}}$, the external forces $7.5 \mathrm{kN}$, $5 \mathrm{kN}$ and $2.5 \mathrm{kN}$ are applied at the nodes (a), (b), and (c), respectively, in the negative direction of $y$-axis. The uncertainty model of $\boldsymbol{f},(5)$, is defined as discussed in Example 6.3 with $\bar{f}_{0}=1 \mathrm{kN}$. Consequently, the external forces are running through the circles depicted with the dotted lines in Figure 22. Note that uncertain forces may possibly exist only at the nodes (a)-(c), and any two sets of the uncertain loads have no relation.

We investigate the displacement of the node (a), which is denoted by $\left(u_{x}, u_{y}\right)$. We put $\alpha=1.0$ in (5). For the amplitudes in the $x$ - and $y$-th directions, we obtain the bounds of the moduli and phase angles, which are shown in Figure 23 for various frequency of the driving load. Figure 24 depicts the bounds for the distribution of the vector $\left(\left|u_{x}\right|,\left|u_{y}\right|\right)$. It is observed from Figure 23 and Figure 25 that the obtained bounds are confirmed to be conservative and very tight.

\section{Conclusions}

We have proposed tractable formulations for computing confidential bounds for the dynamic steadystate behaviors of a structure subjected to uncertain driving loads. We considered non-stochastic uncertainties of amplitudes of harmonic driving loads, and estimate conservative bounds for structural responses at the steady-state.

We have formulated the bound detection problems as optimization problems for finding the minimal bounds of various characteristic amounts of dynamic response, including the modulus and phase angle of the complex amplitude of the displacement and stress. By using the quadraticembedding of the uncertain parameters, and applying the $\mathcal{S}$-lemma, we constructed a numerically tractable problem which approximates the bound detection problem, and provides a conservative bound for the dynamic response. The obtained conservative problems are shown to be either an SDP (semidefinite programming) problem or an SOCP (second-order cone programming) problem, both of which are convex optimization problems, and can be solved very effectively by using existing well-developed software based on the primal-dual interior-point method. 


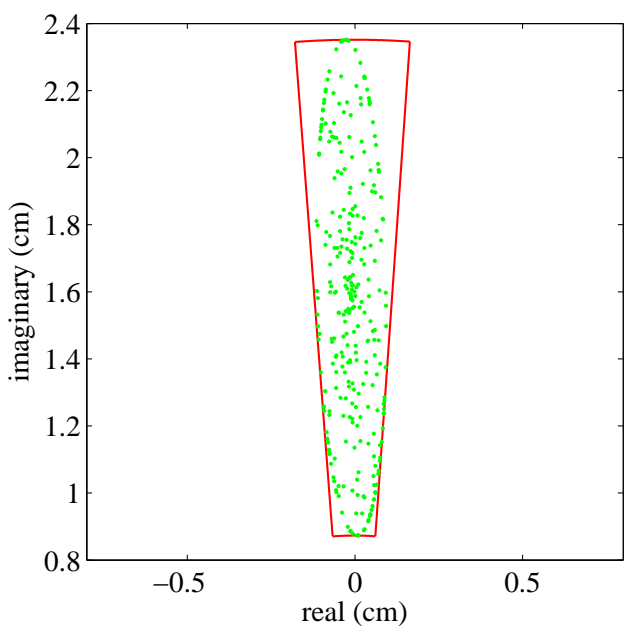

(i) $x$-direction $\left(\omega=\omega_{1}^{0}\right)$

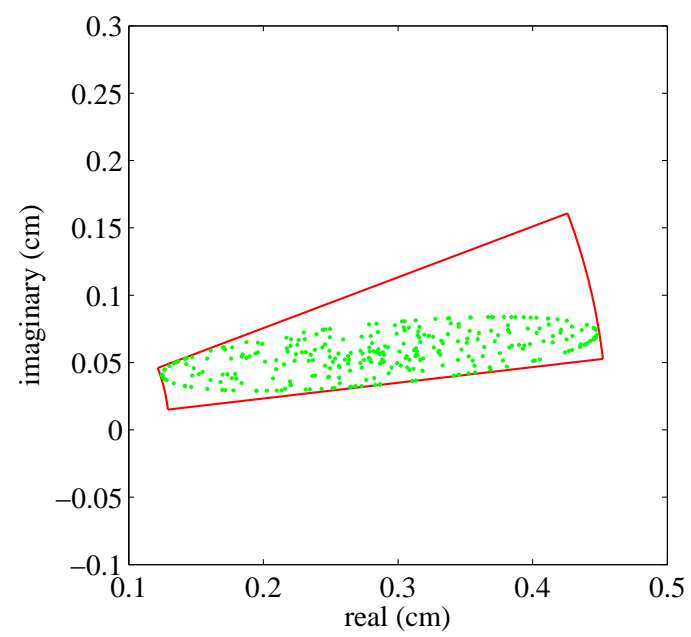

(iii) $x$-direction $\left(\omega=1.1 \omega_{1}^{0}\right)$

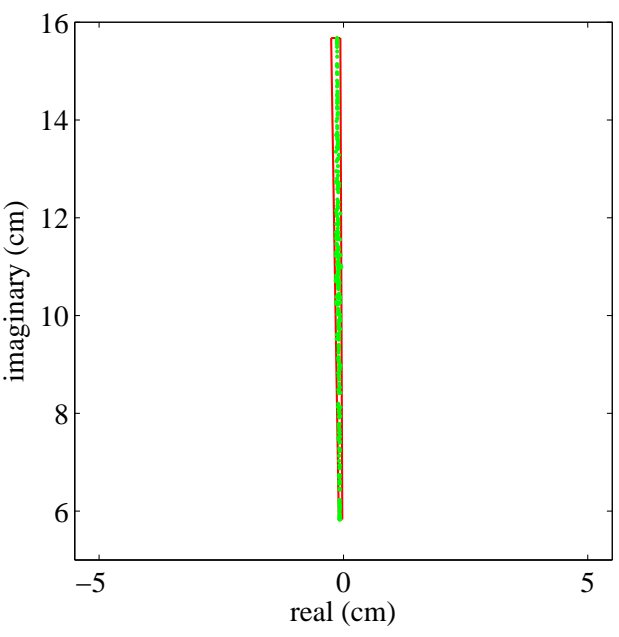

(ii) $y$-direction $\left(\omega=\omega_{1}^{0}\right)$

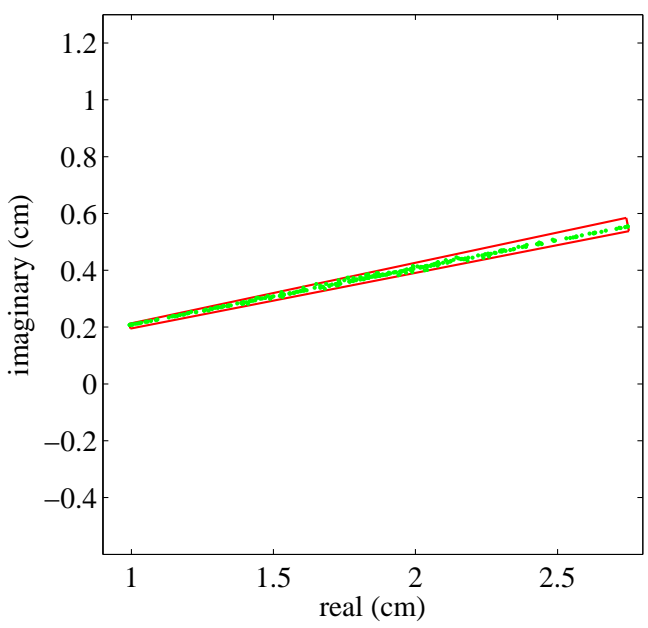

(iv) $y$-direction $\left(\omega=1.1 \omega_{1}^{0}\right)$

Figure 23: Bounds and random samples for the complex amplitudes of the displacements at the node (a) of the model defined in Figure 22.

In the numerical examples, it has been shown that confidence bounds for dynamic responses can be obtained effectively by using the primal-dual interior-point method. We have also illustrated through numerical examples that the bounds provided by the proposed SDP and SOCP problems are sufficiently tight even for a moderately large magnitude of uncertainty. 


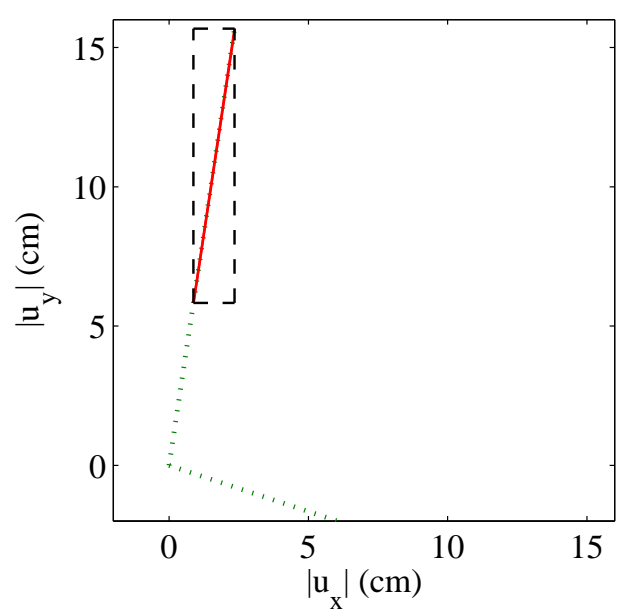

(i) $\omega=\omega_{1}^{0}$

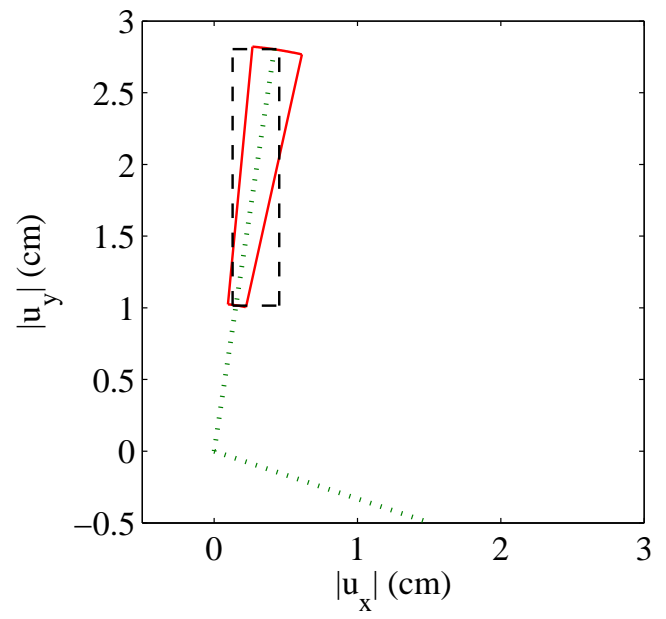

(ii) $\omega=1.1 \omega_{1}^{0}$

Figure 24: Bounds for the oscillation of the node (a) of the 29-bar truss defined in Figure 22. '': bounds obtained by solving (44), (45), (50), and (53); '- -': bounds obtained by solving (19) and $(21) ; ' \ldots$ ': directions of eigenmodes without damping.

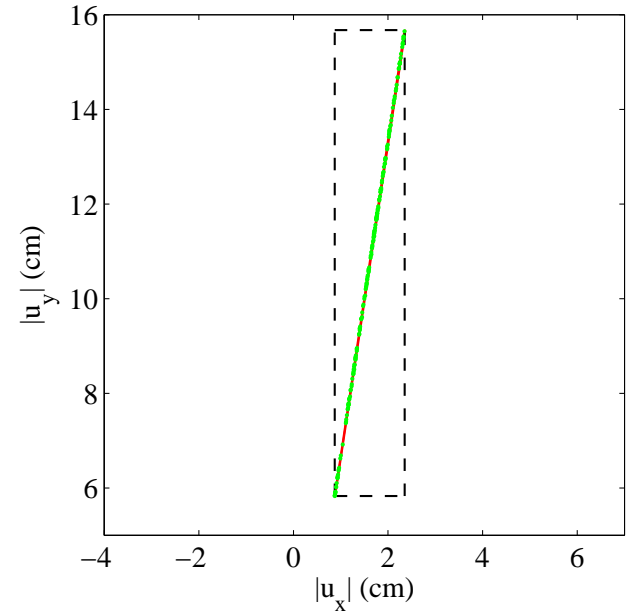

(i) $\omega=\omega_{1}^{0}$

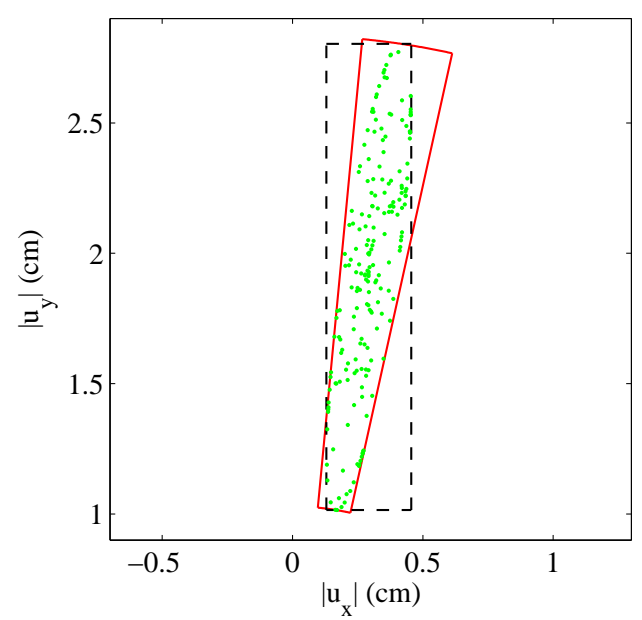

(ii) $\omega=1.1 \omega_{1}^{0}$

Figure 25: Random samples for the oscillation of the node (a) of the 29-bar truss defined in Figure 22. 


\section{Appendix A. Proofs of technical results}

\section{A.1 For section 3.1}

\section{A.1.1 Proof of Proposition 3.1}

By using $G$ defined in (13), the problem (7) is equivalently rewritten as

$$
r_{\max }=\max _{\boldsymbol{v}}\{\|G \boldsymbol{v}\|: \boldsymbol{v} \in \mathcal{V}\} .
$$

Since we assume that $\mathcal{F}$ defined in (5) is bounded, $\mathcal{V}$ is also bounded. Hence, $\|G \boldsymbol{v}\|$ is bounded above, which implies that there exists an $r^{\prime} \in \mathbb{R}$ satisfying $r^{\prime} \geq\|G \boldsymbol{v}\|(\forall \boldsymbol{v} \in \mathcal{V})$. Here, $r^{\prime}$ is regarded as an upper bound of $r_{\max }$ in (57), and hence $r^{\prime} \geq r_{\max }$. Moreover, it is easy to see that $r_{\max }$ satisfies $r_{\max } \geq\|G \boldsymbol{v}\|(\forall \boldsymbol{v} \in \mathcal{V})$, i.e. $r_{\max }$ is a feasible solution of (14). Consequently, $r_{\max }$ is the minimum value of $r^{\prime}$ when $r^{\prime}$ is an upper bound of $\|G \boldsymbol{v}\|$ over $\mathcal{V}$. This is (14).

\section{A.1.2 Proof of Proposition 3.3}

The following two lemmas are used for the proof of Proposition 3.3.

Lemma A.1. Let $Q \in \mathcal{S}^{n}, \boldsymbol{p} \in \mathbb{R}, \gamma \in \mathbb{R}$, and $\boldsymbol{x} \in \mathbb{R}^{n}$. Then the following two conditions are equivalent:

$$
\begin{aligned}
& \text { (a) : } \quad \forall \boldsymbol{x} \in \mathbb{R}^{n}:\left[\begin{array}{l}
\boldsymbol{x} \\
1
\end{array}\right]^{\mathrm{T}}\left[\begin{array}{cc}
Q & \boldsymbol{p} \\
\boldsymbol{p}^{\mathrm{T}} & \gamma
\end{array}\right] Q\left[\begin{array}{l}
\boldsymbol{x} \\
1
\end{array}\right] \geq 0 ; \\
& \text { (b) : }\left[\begin{array}{cc}
Q & \boldsymbol{p} \\
\boldsymbol{p}^{\mathrm{T}} & \gamma
\end{array}\right] \succeq O
\end{aligned}
$$

Proof. The implication from (b) to (a) is trivial. We show that (a) implies (b) by the contradiction. Suppose that (b) does not holds, i.e. we assume that there exist $\boldsymbol{x}^{\prime} \in \mathbb{R}^{n}$ and $\xi \in \mathbb{R}$ satisfying

$$
\left[\begin{array}{c}
\boldsymbol{x}^{\prime} \\
\xi
\end{array}\right]^{\mathrm{T}}\left[\begin{array}{ll}
Q & \boldsymbol{p} \\
\boldsymbol{p}^{\mathrm{T}} & \gamma
\end{array}\right]\left[\begin{array}{l}
\boldsymbol{x}^{\prime} \\
\xi
\end{array}\right]<0 .
$$

If $\xi \neq 0$, then $(58)$ is reduced to

$$
\left[\begin{array}{c}
\boldsymbol{x}^{\prime} / \xi \\
1
\end{array}\right]^{\mathrm{T}}\left[\begin{array}{cc}
Q & \boldsymbol{p} \\
\boldsymbol{p}^{\mathrm{T}} & \gamma
\end{array}\right]\left[\begin{array}{c}
\boldsymbol{x}^{\prime} / \xi \\
1
\end{array}\right]<0,
$$

which contradicts the condition (a). Alternatively, if $\xi=0$, then (58) is reduced to

$$
\boldsymbol{x}^{\prime \mathrm{T}} Q \boldsymbol{x}^{\prime}<0
$$

Put $\boldsymbol{x}=\eta \boldsymbol{x}^{\prime}$, and the left-hand side of (a) is reduced to

$$
\left(\boldsymbol{x}^{\mathrm{T}} Q \boldsymbol{x}^{\prime}\right) \eta^{2}+2\left(\boldsymbol{p}^{\mathrm{T}} \boldsymbol{x}^{\prime}\right) \eta+\gamma,
$$

which is regarded as a function of $\eta$. The condition (59) implies that (60) is not bounded below, from which it follows that there exists $\eta$ such that (60) becomes negative. Thus, we see the contradiction to (a). 
Lemma A.2 (S-lemma). Let $f_{0}, f_{1}, \ldots, f_{m}: \mathbb{R}^{n} \rightarrow \mathbb{R}$ be quadratic functions. The implication

$$
f_{1}(\boldsymbol{x}) \geq 0, \ldots, f_{m}(\boldsymbol{x}) \geq 0 \quad \Rightarrow \quad f_{0}(\boldsymbol{x}) \geq 0
$$

holds if there exist a vector $\boldsymbol{\tau}=\left(\tau_{i}\right) \in \mathbb{R}^{m}$ satisfying

$$
\begin{aligned}
& \forall \boldsymbol{x} \in \mathbb{R}^{n}: \quad f_{0}(\boldsymbol{x}) \geq \sum_{i=1}^{m} \tau_{i} f_{i}(\boldsymbol{x}), \\
& \tau_{1}, \ldots, \tau_{m} \geq 0 .
\end{aligned}
$$

Proof. See Boyd et al. [8, section 2.6.3] and the references therein.

Proof of Proposition 3.3 For simplicity we write

$$
\begin{aligned}
& q_{i}(\boldsymbol{x})=\left[\begin{array}{l}
\boldsymbol{x} \\
1
\end{array}\right]^{\mathrm{T}} Q_{i}\left[\begin{array}{l}
\boldsymbol{x} \\
1
\end{array}\right], \quad i=0,1, \ldots, m, \\
& p_{j}(\boldsymbol{x})=\left[\begin{array}{c}
\boldsymbol{x} \\
1
\end{array}\right]^{\mathrm{T}} P_{j}\left[\begin{array}{c}
\boldsymbol{x} \\
1
\end{array}\right], \quad j=1, \ldots, k .
\end{aligned}
$$

Observe that the quadratic equation $p_{j}(\boldsymbol{x})=0$ in (a) is equivalent to quadratic inequalities

$$
p_{j}(\boldsymbol{x}) \geq 0, \quad-p_{j}(\boldsymbol{x}) \geq 0 .
$$

It follows from Lemma A.2 that the implication $(\mathrm{a}) \Rightarrow(\mathrm{b})$ holds if there exist $\tau_{1}, \ldots, \tau_{m}, \rho_{m+1}^{+}, \ldots, \rho_{m+k}^{+}$, and $\rho_{m+1}^{-}, \ldots, \rho_{m+k}^{-}$satisfying

$$
\begin{aligned}
& \forall \boldsymbol{x} \in \mathbb{R}^{n}: \quad q_{0}(\boldsymbol{x}) \geq \sum_{i=1}^{m} \tau_{i} q_{i}(\boldsymbol{x})+\sum_{j=1}^{k} \rho_{j}^{+} p_{j}(\boldsymbol{x})+\sum_{j=1}^{k} \rho_{j}^{-}\left(-p_{j}(\boldsymbol{x})\right), \\
& \tau_{1}, \ldots, \tau_{m} \geq 0, \rho_{1}^{+}, \ldots, \rho_{k}^{+} \geq 0, \rho_{1}^{-}, \ldots, \rho_{k}^{-} \geq 0 .
\end{aligned}
$$

By putting $\sigma_{j}=\rho_{j}^{+}-\rho_{j}^{-}(j=1, \ldots, k)$, this condition is equivalently rewritten as

$$
\begin{aligned}
& \forall \boldsymbol{x} \in \mathbb{R}^{n}: \quad q_{0}(\boldsymbol{x}) \geq \sum_{i=1}^{m} \tau_{i} q_{i}(\boldsymbol{x})+\sum_{j=1}^{k} \sigma_{j} p_{j}(\boldsymbol{x}), \\
& \tau_{1}, \ldots, \tau_{m} \geq 0 .
\end{aligned}
$$

The assertion of this proposition is obtained by applying Lemma A.1 to (61).

\section{A.1.3 Proof of Proposition 3.4}

It follows from (5) and (12) that $\boldsymbol{v} \in \mathcal{V}$ if and only if

$$
\begin{aligned}
& S_{1} \boldsymbol{v}=\tilde{\boldsymbol{f}}+F_{0} \boldsymbol{\zeta}, \quad \alpha \geq\left\|T_{j} \boldsymbol{\zeta}\right\|, \quad j=1, \ldots, \ell, \\
& S_{2} \boldsymbol{v}=\mathbf{0} .
\end{aligned}
$$

Since $F_{0}$ is regular, (62) is rewritten as

$$
T_{j} \boldsymbol{\zeta}=T_{j} F_{0}^{-1}\left(S_{1} \boldsymbol{v}-\tilde{\boldsymbol{f}}\right), \quad \alpha \geq\left\|T_{j} \boldsymbol{\zeta}\right\|, \quad j=1, \ldots, \ell .
$$


For each $j=1, \ldots, \ell$, we see that

$$
\alpha \geq\left\|T_{j} \boldsymbol{\zeta}\right\| \quad \Leftrightarrow \quad \alpha^{2}-\left\|T_{j} \boldsymbol{\zeta}\right\|^{2} \geq 0
$$

holds. From this observation it follows that (64) is equivalent to the quadratic inequalities

$$
\alpha^{2}-\left\|T_{j} F_{0}^{-1}\left(S_{1} \boldsymbol{v}-\tilde{\boldsymbol{f}}\right)\right\|^{2} \geq 0, \quad j=1, \ldots, \ell,
$$

which corresponds to (15). The equation (63) is equivalent to the quadratic equality

$$
\left\|S_{2} \boldsymbol{v}\right\|^{2}=0
$$

which corresponds to (16).

\section{A.1.4 Proof of Proposition 3.5}

We start with observing that the condition (17) is equivalent to the implication

$$
\boldsymbol{v} \in \mathcal{V} \quad \Rightarrow \quad r \geq\|G \boldsymbol{v}\|
$$

Here, the inequality on the right-hand side of (65) is reduced to

$$
\left[\begin{array}{l}
\boldsymbol{v} \\
1
\end{array}\right]^{\mathrm{T}}\left[\begin{array}{cc}
-G^{\mathrm{T}} G & \mathbf{0} \\
\mathbf{0}^{\mathrm{T}} & r^{2}
\end{array}\right]\left[\begin{array}{l}
\boldsymbol{v} \\
1
\end{array}\right] \geq 0 .
$$

Note that the right-hand side of (65) is equivalent to (66) and $r \geq 0$.

Recall that, in Proposition 3.4, the left-hand side of (65) is reduced to quadratic inequalities. Consequently, the implication (65) can be rewritten equivalently as the following implication:

$$
\begin{gathered}
{\left[\begin{array}{l}
\boldsymbol{v} \\
1
\end{array}\right]^{\mathrm{T}} \Psi_{j}\left[\begin{array}{l}
\boldsymbol{v} \\
1
\end{array}\right]^{2} \geq 0(j=1, \ldots, \ell), \quad\left[\begin{array}{l}
\boldsymbol{v} \\
1
\end{array}\right]^{\mathrm{T}} \Theta\left[\begin{array}{l}
\boldsymbol{v} \\
1
\end{array}\right]=0} \\
\Rightarrow\left[\begin{array}{l}
\boldsymbol{v} \\
1
\end{array}\right]^{\mathrm{T}}\left[\begin{array}{cc}
-G^{\mathrm{T}} G & \mathbf{0} \\
\mathbf{0}^{\mathrm{T}} & r^{2}
\end{array}\right]\left[\begin{array}{l}
\boldsymbol{v} \\
1
\end{array}\right] \geq 0,
\end{gathered}
$$

where $r \geq 0$. Then the assertion of Proposition 3.5 follows from Proposition 3.3.

\section{A.1.5 Proof of Proposition 3.6}

Recall Proposition 3.1, i.e. $r_{\max }$ is equivalent to the optimal value of the problem (14). Now we observe that the problem (19) is obtained by replacing the constraint condition, (17), of the problem (14) with (18). Hence, the assertion of Proposition 3.6 follows immediately from Proposition 3.5.

\section{A.2 For section 3.2}

\section{A.2.1 Proof of Proposition 3.7}

By using $G$ defined by (13), we see that (20) is equivalently rewritten as

$$
r_{\min }=\min _{\boldsymbol{v}}\{\|G \boldsymbol{v}\|: \boldsymbol{v} \in \mathcal{V}\}
$$


By introducing an auxiliary variable $t$ which is equivalent to $\|G \boldsymbol{v}\|$, and by substituting the definition $(12)$ of $\mathcal{V}$, the problem $(67)$ is reduced to

$$
\left.\begin{array}{ll}
\min _{t, \boldsymbol{v}, \boldsymbol{\zeta}} & t \\
\text { s.t. } & t=\|G \boldsymbol{v}\|, \\
& S_{1} \boldsymbol{v}=\tilde{\boldsymbol{f}}+F_{0} \boldsymbol{\zeta}, \\
& S_{2} \boldsymbol{v}=\mathbf{0}, \\
& \alpha \geq\left\|T_{j} \boldsymbol{\zeta}\right\|, \quad j=1, \ldots, \ell,
\end{array}\right\}
$$

without changing the optimal value. Since the problem (68) is the minimization of $t$, we can replace the constraint condition $r=\|G \boldsymbol{v}\|$ with $r \geq\|G \boldsymbol{v}\|$, which results in (21).

\section{A.3 For section 3.3}

\section{A.3.1 Proof of Proposition 3.10}

Since $0<\theta_{\max }<\pi$, we may restrict ourselves to $u_{q}$ satisfying $\operatorname{Im} u_{q} \neq 0$ without loss of generality. It is easy to see that if $a_{2} \in \mathbb{R}$ and $u_{q} \in \mathbb{C}$ satisfy

$$
\left[\begin{array}{ll}
1 & a_{2}
\end{array}\right]\left[\begin{array}{c}
\operatorname{Re} u_{q} \\
\operatorname{Im} u_{q}
\end{array}\right]=0
$$

then the relation

$$
a_{2}=-\frac{\operatorname{Re} u_{q}}{\operatorname{Im} u_{q}}=-\frac{1}{\tan \operatorname{Arg} u_{q}}
$$

holds. Hence, (23) is reduced to

$$
-\frac{1}{\tan \theta_{\max }}=\max _{\boldsymbol{a}}\left\{\boldsymbol{e}_{2}^{\mathrm{T}} \boldsymbol{a}: \boldsymbol{a}^{\mathrm{T}} G \boldsymbol{v}=0, \boldsymbol{v} \in \mathcal{V}, \boldsymbol{e}_{1}^{\mathrm{T}} \boldsymbol{a}=1\right\}
$$

Moreover, if $a_{2}$ and $u_{q}$ satisfy

$$
\left[\begin{array}{ll}
1 & a_{2}
\end{array}\right]\left[\begin{array}{l}
\operatorname{Re} u_{q} \\
\operatorname{Im} u_{q}
\end{array}\right] \geq 0
$$

then the inequality

$$
a_{2} \geq-\frac{1}{\tan \operatorname{Arg} u_{q}}
$$

holds. Hence, (70) implies that $a_{2}$ is an upper bound for $-1 / \tan \operatorname{Arg} u_{q}$. From this observation it follows that if $a_{2}$ satisfies

$$
\left[\begin{array}{ll}
1 & a_{2}
\end{array}\right] G \boldsymbol{v} \geq 0, \quad \forall \boldsymbol{v} \in \mathcal{V}
$$

then $a_{2}$ corresponds to an upper bound for $-1 / \tan \operatorname{Arg} u_{q}(\boldsymbol{f})$ when $\boldsymbol{f}$ is running through $\mathcal{F}$. Consequently, in a manner similar to Proposition 3.1, the maximization problem (69) of $-1 / \tan \operatorname{Arg} u_{q}$ is equivalently rewritten as the minimization problem of the upper bound for $-1 / \tan \operatorname{Arg} u_{q}$, which concludes the proof. 


\section{A.3.2 Proof of Proposition 3.11}

We first observe that the condition (27) is equivalent to the following implication:

$$
\boldsymbol{v} \in \mathcal{V} \Rightarrow \boldsymbol{a}^{\mathrm{T}} G \boldsymbol{v} \geq 0
$$

Recall that the condition $\boldsymbol{v} \in \mathcal{V}$ has been embedded into some quadratic inequalities in Proposition 3.4. We easily see that

$$
\boldsymbol{a}^{\mathrm{T}} G \boldsymbol{v} \geq 0
$$

is equivalent to

$$
\left[\begin{array}{l}
\boldsymbol{v} \\
1
\end{array}\right]^{\mathrm{T}}\left[\begin{array}{cc}
O & G^{\mathrm{T}} \boldsymbol{a} \\
\boldsymbol{a}^{\mathrm{T}} G & 0
\end{array}\right]\left[\begin{array}{l}
\boldsymbol{v} \\
1
\end{array}\right] \geq 0
$$

Then the assertion follows from Proposition 3.3.

\section{A.3.3 Proof of Proposition 3.12}

Recall Proposition 3.10, i.e. $\theta_{\max }$ is equivalent to the optimal value of the problem (26). Observe that the problem (29) is constructed from the problem (26) by replacing the constraint condition (27) with its sufficient condition (28) provided by Proposition 3.11. Let $\left(\boldsymbol{a}^{\prime}, \boldsymbol{w}^{\prime}, s^{\prime}\right)$ denote a feasible solution of the problem (29). From the construction of (29) it follows that $\boldsymbol{a}^{\prime}$ satisfies (27). Hence, by using Proposition 3.10, we obtain the inequality

$$
-\frac{1}{\tan \theta_{\max }} \leq a_{2}^{*}
$$

which concludes the proof.

\section{A.4 For section 4.2}

\section{A.4.1 Proof of Proposition 4.5}

We investigate the constraint condition

$$
\forall \boldsymbol{\mu} \in\left\{\left(\left\|G_{x} \boldsymbol{v}\right\|^{2},\left\|G_{y} \boldsymbol{v}\right\|^{2}\right)^{\mathrm{T}} \mid \boldsymbol{v} \in \mathcal{V}\right\}: \quad \boldsymbol{a}^{\mathrm{T}} \boldsymbol{\mu} \geq 0
$$

in the problem (49). It follows from Proposition 3.4 that the condition $\boldsymbol{\mu} \in\left\{\left(\left\|G_{x} \boldsymbol{v}\right\|^{2},\left\|G_{y} \boldsymbol{v}\right\|^{2}\right)^{\mathrm{T}} \mid\right.$ $\boldsymbol{v} \in \mathcal{V}\}$ in (71) can be reduced to quadratic inequalities in terms of $\boldsymbol{u}$ and $\boldsymbol{\mu}$ as

$$
\begin{aligned}
& {\left[\begin{array}{l}
\boldsymbol{v} \\
1
\end{array}\right]^{\mathrm{T}} \boldsymbol{\Psi}_{j}\left[\begin{array}{l}
\boldsymbol{v} \\
1
\end{array}\right] \geq 0, \quad j=1, \ldots, \ell,} \\
& {\left[\begin{array}{l}
\boldsymbol{v} \\
1
\end{array}\right]^{\mathrm{T}} \Theta\left[\begin{array}{l}
\boldsymbol{v} \\
1
\end{array}\right]=0,} \\
& \mu_{x}^{2}-\left\|G_{x} \boldsymbol{v}\right\|^{2}=0, \quad \mu_{y}^{2}-\left\|G_{y} \boldsymbol{v}\right\|^{2}=0 .
\end{aligned}
$$


which are rewritten as

$$
\begin{aligned}
& {\left[\begin{array}{l}
\boldsymbol{\mu} \\
\boldsymbol{v} \\
1
\end{array}\right]^{\mathrm{T}} \hat{\Psi}_{j}\left[\begin{array}{l}
\boldsymbol{\mu} \\
\boldsymbol{v} \\
1
\end{array}\right] \geq 0, \quad j=1, \ldots, \ell,} \\
& {\left[\begin{array}{l}
\boldsymbol{\mu} \\
\boldsymbol{v} \\
1
\end{array}\right]^{\mathrm{T}} \hat{\Theta}_{i}\left[\begin{array}{l}
\boldsymbol{\mu} \\
\boldsymbol{v} \\
1
\end{array}\right]=0, \quad i=1,2,3 .}
\end{aligned}
$$

The condition $\boldsymbol{a}^{\mathrm{T}} \boldsymbol{\mu} \geq 0$ in (71) is equivalently rewritten as

$$
\left[\begin{array}{c}
\boldsymbol{\mu} \\
\boldsymbol{v} \\
1
\end{array}\right]^{\mathrm{T}}\left[\begin{array}{ccc}
O & O & \boldsymbol{a} \\
O & O & \mathbf{0} \\
\boldsymbol{a}^{\mathrm{T}} & \mathbf{0}^{\mathrm{T}} & 0
\end{array}\right]\left[\begin{array}{l}
\boldsymbol{\mu} \\
\boldsymbol{v} \\
1
\end{array}\right] \geq 0 .
$$

Consequently, the condition (71) is equivalent to the implication

$$
(72) \&(73) \quad \Rightarrow \quad(74) \text {. }
$$

By applying Proposition 3.3 to (75), we see that the implication (75) holds if there exist $\boldsymbol{w} \in \mathbb{R}_{+}^{\ell}$ and $s \in \mathbb{R}^{3}$ satisfying

$$
\left[\begin{array}{ccc}
O & O & \boldsymbol{a} \\
O & O & \mathbf{0} \\
\boldsymbol{a}^{\mathrm{T}} & \mathbf{0}^{\mathrm{T}} & 0
\end{array}\right] \succeq \sum_{j=1}^{\ell} w_{j} \hat{\Psi}_{j}+\sum_{i=1}^{3} s_{i} \hat{\Theta}_{i}
$$

Note that the problem (50) is obtained from the problem (49) by replacing the constraint condition (71) with its sufficient condition (76). Hence, $a_{2}^{*}$ defined in (50) satisfies $a_{2}^{*} \geq-1 /\left(t_{\max }\right)^{2}$, from which we obtain Proposition 4.5.

\section{A.5 For section 6.1}

\section{A.5.1 Proof of Proposition 6.2}

From (5) and (12), and the definitions of $F_{0}^{\dagger}$ and $F_{0}^{\perp}$, we see that $\boldsymbol{v} \in \mathcal{V}$ if and only if $\boldsymbol{v}$ satisfies

$$
\begin{aligned}
& F_{0}^{\dagger}\left(S_{1} \boldsymbol{v}-\tilde{\boldsymbol{f}}\right)=\boldsymbol{\zeta}, \quad \alpha \geq\left\|T_{j} \boldsymbol{\zeta}\right\|, \quad j=1, \ldots, \ell \\
& \left(F_{0}^{\perp}\right)^{\mathrm{T}}\left(S_{1} \boldsymbol{v}-\tilde{\boldsymbol{f}}\right)=\mathbf{0} \\
& S_{2} \boldsymbol{v}=\mathbf{0}
\end{aligned}
$$

The condition (77) is reduced to

$$
\alpha \geq\left\|T_{j} F_{0}^{\dagger}\left(S_{1} \boldsymbol{v}-\tilde{\boldsymbol{f}}\right)\right\|, \quad j=1, \ldots, \ell,
$$

which is rewritten equivalently as (55). Moreover, the linear equations (78) and (79) are equivalently rewritten as (56), which concludes the proof. 


\section{Acknowledgments}

This work is supported by a Grant-in-Aid for Scientific Research from the Ministry of Education, Culture, Sports, Science and Technology of Japan.

\section{References}

[1] Alefeld, G. and Mayer, G., Interval analysis: theory and applications, Journal of Computational and Applied Mathematics, 121 (2000) 421-464.

[2] Alizadeh, F. and Goldfarb, D., Second-order cone programming. Mathematical Programming, B95 (2003) 3-51.

[3] Au, F.T.K., Cheng, Y.S., Tham, L.G., and Zheng, G.W., Robust design of structures using convex models, Computers \& Structures, 81 (2003) 2611-2619.

[4] Ben-Haim, Y., Information-gap Decision Theory: Decisions under Severe Uncertainty, (2nd ed.), Academic Press, London, 2006.

[5] Ben-Haim, Y. and Elishakoff, I., Convex Models of Uncertainty in Applied Mechanics, Elsevier, New York, 1990.

[6] Ben-Tal, A., El Ghaoui, L., and Nemirovski, A., Robustness, in: H. Wolkowicz, R. Saigal, L. Vandenberghe (eds.), Handbook of Semidefinite Programming - Theory, Algorithms, and Applications, Kluwer, Dordrecht, the Netherlands, 2000, pp. 139-162.

[7] Ben-Tal, A. and Nemirovski, A., Robust optimization - methodology and applications, Mathematical Programming, B92 (2002) 453-480.

[8] Boyd, S., El Ghaoui, L., Feron, E., and Balakrishnan, V., Linear Matrix Inequalities in System and Control Theory, SIAM, Philadelphia, 1994.

[9] Calafiore, G. and El Ghaoui, L., Ellipsoidal bounds for uncertain linear equations and dynamical systems, Automatica, 40 (2004) 773-787.

[10] Chen, S., Lian, H., and Yang, X., Interval eigenvalue analysis for structures with interval parameters, Finite Elements in Analysis and Design, 39 (2003) 419-431.

[11] Chen, S., Qiu, Z., and Song, D., A new method for computing the upper and lower bounds on frequencies of structures with interval parameters, Mechanics Research Communications, 22 (1995) 431-439.

[12] De Gersem, H., Moens, D., Desmet, W., and Vandepitte, D., Interval and fuzzy dynamic analysis of finite element models with superelements, Computers \& Structures, 85 (2007) 304-319.

[13] Dimarogonas, A.D., Interval analysis of vibrating systems, Journal of Sound and Vibration, 183 (1995) 739-749. 
[14] Gao, W., Interval natural frequency and mode shape analysis for truss structures with interval parameters, Finite Elements in Analysis and Design, 42 (2006) 471-477.

[15] El-Gebeily, M.A., Abu-Baker, Y., and Elginde, M.B., The generalized eigenvalue problem for tridiagonal symmetric interval matrices, International Journal of Control, 72 (1999) 531-535.

[16] Guo, X., Bai, W., and Zhang, W., Extreme structural response analysis of truss structures under material uncertainty via linear mixed 0-1 programming, International Journal for Numerical Methods in Engineering, (2008) DOI: 10.1002/nme.2298.

[17] Helmberg, C., Semidefinite programming, European Journal of Operational Research, 137 (2002) 461-482.

[18] Kanno, Y. and Takewaki, I., Confidence ellipsoids for static response of trusses with load and structural uncertainties, Computer Methods in Applied Mechanics and Engineering, 196 (2006) 393-403.

[19] Kanno, Y. and Takewaki, I., Robustness analysis of trusses with separable load and structural uncertainties, International Journal of Solids and Structures, 43 (2006) 2646-2669.

[20] Kanno, Y. and Takewaki, I., Worst-case plastic limit analysis of trusses under uncertain loads via mixed 0-1 programming, Journal of Mechanics of Materials and Structures, 2 (2007) 245273.

[21] Kanno, Y. and Takewaki, I., Ellipsoidal bounds for static response of framed structures against interactive uncertainties, Interaction and Multiscale Mechanics: an International Journal, $\mathbf{1}$ (2007) 103-121.

[22] Kanno, Y. and Takewaki, I., Semidefinite programming for uncertain linear equations in static analysis of structures, Computer Methods in Applied Mechanics and Engineering, 198 (2008) $102-115$.

[23] Kanno, Y. and Takewaki, I., Semidefinite programming for dynamic steady-state analysis of structures under uncertain harmonic loads, Mathematical Engineering Technical Report, METR 2008-35, Dept. of Mathematical Informatics, University of Tokyo, Japan, August 2008.

[24] Leng, H. and He, Z., Computing eigenvalue bounds of structures with uncertain-but-nonrandom parameters by a method based on perturbation theory, Communications in Numerical Methods in Engineering, 23 (2007) 973-982.

[25] Matsuda, Y. and Kanno, Y., Robustness analysis of structures based on plastic limit analysis with uncertain loads, Journal of Mechanics of Materials and Structures, 3 (2008) 213-242.

[26] Modares, M., Mullen, R.L., and Muhanna, R.L., Natural frequencies of a structure with bounded uncertainty, Journal of Engineering Mechanics (ASCE), 132 (2006) 1363-1371.

[27] Moens, D. and Vandepitte, D., A fuzzy finite element procedure for the calculation of uncertain frequency-response functions of damped structures: Part 1 - Procedure, Journal of Sound and Vibration, 288 (2005) 431-462. 
[28] Moens, D. and Vandepitte, D., A survey of non-probabilistic uncertainty treatment in finite element analysis, Computer Methods in Applied Mechanics and Engineering, 194 (2005) 12571555 .

[29] Muhanna, R.L. and Mullen, R.L., Uncertainty in mechanics problems - interval-based approach, Journal of Engineering Mechanics (ASCE), 127 (2001) 557-566.

[30] Neumaier, A. and Pownuk, A., Linear systems with large uncertainties, with applications to truss structures, Reliable Computing, 13 (2007) 149-172.

[31] Pantelides, C.P. and Ganzerli, S., Design of trusses under uncertain loads using convex models, Journal of Structural Engineering (ASCE), 124 (1998) 318-329.

[32] Pantelides, C.P. and Tzan, S.-R., Convex model for seismic design of structures-I: Analysis, Earthquake Engineering and Structural Dynamics, 25 (1996) 927-944.

[33] Qiu, Z., Chen, S.H., and Elishakoff, I., Natural frequencies of structures with uncertain but nonrandom parameters, Journal of Optimization Theory and Applications, 86 (1995) 669-683.

[34] Qiu, Z., Elishakoff, I., and Starnes, J.H., Jr., The bound set of possible eigenvalues of structures with uncertain but non-random parameters, Chaos, Solitons 85 Fractals, 7 (1996) 1845-1857.

[35] Qiu, Z. and Wang, X., Comparison of dynamic response of structures with uncertain-butbounded parameters using non-probabilistic interval analysis method and probabilistic approach, International Journal of Solids and Structures, 40 (2003) 5423-5439.

[36] Schuëller, G.I., Developments in stochastic structural mechanics, Archive of Applied Mechanics, 75 (2006) 755-773.

[37] Sim, J.S., Qiu, Z., and Wang, X., Modal analysis of structures with uncertain-but-bounded parameters via interval analysis, Journal of Sound and Vibration, 303 (2007) 29-45.

[38] Sturm, J.F., Using SeDuMi 1.02, a MATLAB toolbox for optimization over symmetric cones, Optimization Methods and Software, 11/12 (1999) 625-653.

[39] Takewaki, I. and Ben-Haim, Y., Info-gap robust design with load and model uncertainties, Journal of Sound and Vibration, 288 (2005) 551-570.

[40] Tzan, S.-R. and Pantelides, C.P., Convex models for impulsive response of structures, Journal of Engineering Mechanics (ASCE), 122 (1996) 521-529.

[41] Zang, C., Friswell, M.I., and Mottershead J.E., A review of robust optimal design and its application in dynamics, Computers \& Structures, 83 (2005) 315-326.

[42] Using MATLAB, The MathWorks, Inc., Natick, 2002. 